\title{
Synthesis of Polysubstituted Germoles and Benzogermoles Using A Substoichiometric Amount of Diisobutylaluminum Hydride
}

\author{
Ko Kojima, Seiya Uchida, Hidenori Kinoshita, ${ }^{*}$ and Katsukiyo Miura
}

Department of Applied Chemistry, Graduate School of Science and Engineering, Saitama University, 255

Shimo-ohkubo, Sakura-ku, Saitama 338-8570, Japan

E-mail:hkino@mail.saitama-u.ac.jp

Supporting Information

Table of Contents

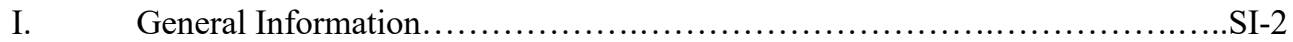

II. Experimental Procedures and Characterization Data...........................SI-3

III. X-ray Crystallographic Data for 4e (CCDC 2075032).......................

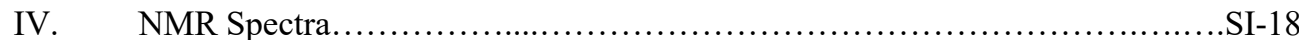




\section{General Information}

${ }^{1} \mathrm{H}$ NMR spectra were recorded at 300, 400, and $500 \mathrm{MHz}$ (Bruker AVANCE 300, Bruker AVANCE 400 cryo, and AVANCE 500) at ambient temperature with $\mathrm{CDCl}_{3} .{ }^{13} \mathrm{C}$ NMR spectra were recorded at 75 , 100 , and $125 \mathrm{MHz}$ at ambient temperature with $\mathrm{CDCl}_{3}$ as solvent. Chemical shifts are reported in parts per million relative to $\mathrm{CHCl}_{3}\left(\delta 7.26 \mathrm{ppm}\right.$ for $\left.{ }^{1} \mathrm{H} \mathrm{NMR}\right)$ and $\mathrm{CDCl}_{3}\left(\delta 77.0 \mathrm{ppm}\right.$ for $\left.{ }^{13} \mathrm{C} \mathrm{NMR}\right)$. Data for ${ }^{1} \mathrm{H}$ NMR are reported as follows: chemical shift, multiplicity $(\mathrm{s}=$ singlet, br.s $=$ broad singlet, $\mathrm{d}=$ doublet, $\mathrm{t}=$ triplet, $\mathrm{q}=$ quartet, quint $=$ quintet, $\mathrm{sept}=$ septet, $\mathrm{m}=$ multiplet $)$, coupling constants $(J$, reported as values in hertz (Hz)), and integration. Infrared spectra were recorded on JASCO FT/IR 4700 spectrophotometer. High-resolution mass spectra were obtained on a JMS700AM (JEOL) in the Comprehensive Analysis Center for Science, Saitama University. Analytical thin layer chromatography was performed using 0.25 $\mathrm{mm}$ silica gel 60-F plates (MERCK). Chromatography was performed using silica gel $60 \mathrm{~N}$ (spherical, neutral, 63-210 mm, KANTO CHEMICAL CO., INC.). Melting point was measured with a MICRO MELTING POINT APPARATUS (YANAGIMOTO SEISAKUSHO). X-ray single crystal structural analysis was conducted on Bruker SMAERT APEX II Ultra.

All reactions were carried out in oven-dried glassware under argon atmosphere. A silicon oil bath was used for the reactions that were required heating. After silica-gel column chromatography, several substitutes were purified with recycling preparative HPLC with $\mathrm{CHCl}_{3}$ (LC-9101, Japan Analytical Industry Co., Ltd.) and collected only pure fraction to remove a mixture of unidentified compounds. Yields refer to chromatographically and spectroscopically pure materials, unless otherwise stated. Octane was dried over Na. THF (super dehydrated and stabilizer free) was purchased from FUJIFILM Wako Pure Chemical Corporation and used as supplied. All other reagents and solvents were used as supplied by Merck Sigma-Aldrich, Tokyo Chemical Industry CO., LTD., FUJIFILM Wako Pure Chemical Corporation, KANTO CHEMICAL CO., INC., and Junsei Chemical Co., Ltd. 


\section{Experimental Procedures and Characterization Data}

(Z)-1-(Trimethylsilyl)-4-(diphenylgermyl)-3-propylhept-3-en-1-yne (1a).

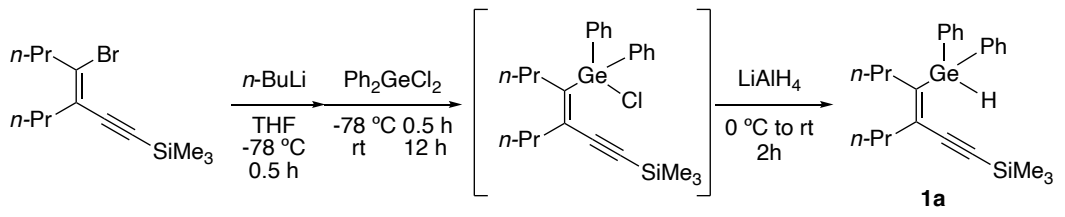

$n$-BuLi (2.64 M in hexane, $1.21 \mathrm{~mL}, 3.19 \mathrm{mmol})$ was added to a THF solution of (Z)-4-brormo-5-[2(trimethylsilyl)ethynyl]oct-4-ene ${ }^{1}(873 \mathrm{mg}, 3.04 \mathrm{mmol})$ at $-78^{\circ} \mathrm{C}$. The mixture was stirred for $0.5 \mathrm{~h}$ at that temperature. Dichlorodiphenylgermane $(0.640 \mathrm{~mL}, 3.04 \mathrm{mmol})$ was added to the mixture at $-78{ }^{\circ} \mathrm{C}$. The resultant mixture was stirred for $1 \mathrm{~h}$ at that temperature and then stirred at room temperature for $12 \mathrm{~h}$. $\mathrm{LiAlH}_{4}(115 \mathrm{mg}, 3.04 \mathrm{mmol})$ was added to the reaction mixture at $0{ }^{\circ} \mathrm{C}$. The mixture was stirred at room temperature for $2 \mathrm{~h}$. After quenching with water, the aqueous mixture was extracted with hexane $(20 \mathrm{~mL})$ three times. The combined organic layer was dried over $\mathrm{Na}_{2} \mathrm{SO}_{4}$ and concentrated. The residue was purified by silica-gel column chromatography (hexane). The title compound was obtained as colorless liquid (862 mg, $1.98 \mathrm{mmol}, 65 \%$ yield). ${ }^{1} \mathrm{H}$ NMR $\left(300 \mathrm{MHz}, \mathrm{CDCl}_{3}\right) \delta$ 7.52-7.56 (m, 4H), 7.32-7.35 (m, 6H), $5.56(\mathrm{~s}$, $1 \mathrm{H}), 2.26-2.32(\mathrm{~m}, 2 \mathrm{H}), 2.18-2.25(\mathrm{~m}, 2 \mathrm{H}), 1.59-1.67(\mathrm{~m}, 2 \mathrm{H}), 1.18-1.26(\mathrm{~m}, 2 \mathrm{H}), 0.97(\mathrm{t}, J=7.2 \mathrm{~Hz}, 3 \mathrm{H})$, $0.72(\mathrm{t}, J=7.2 \mathrm{~Hz}, 3 \mathrm{H}),-0.11(\mathrm{~s}, 9 \mathrm{H}) ;{ }^{13} \mathrm{C} \mathrm{NMR}\left(75 \mathrm{MHz}, \mathrm{CDCl}_{3}\right) \delta 147.2,136.4,135.2,134.4,128.6$, 127.9, 107.1, 96.7, 35.1, 34.2, 22.9, 22.0, 14.0, 13.9, -0.5; IR (neat) 3068, 2959, 2141, 2041, $1432 \mathrm{~cm}^{-1}$; HRMS (EI) m/z: [M] $]^{+}$Calcd for $\mathrm{C}_{25} \mathrm{H}_{34} \mathrm{GeSi} 436.1642$; Found 436.1648.

(Z)-3-Ethyl-1-(trimethylsilyl)-4-(diphenylgermyl)-oct-3-en-1-yne (1b).

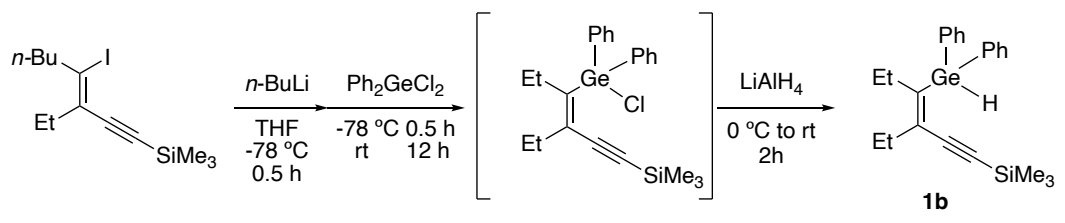

The title compound was synthesized by using (Z)-3-ethyl-4-iodo-1-trimethylsilyloct-3-en-1-yne (859 mg, $2.52 \mathrm{mmol})$, which was synthesized by the reported method, ${ }^{2}$ and dichlorodiphenylgermane $(0.530 \mathrm{~mL}$, $2.52 \mathrm{mmol}$ ) in the same manner as described for the preparation of 1a. Colorless liquid (only pure fraction, $461 \mathrm{mg}, 1.06 \mathrm{mmol}, 42 \%$ yield); ${ }^{1} \mathrm{H}$ NMR $\left(300 \mathrm{MHz}, \mathrm{CDCl}_{3}\right) \delta$ 7.56-7.52 (m, 4H), 7.36-7.30 (m, 6H), 5.57 $(\mathrm{s}, 1 \mathrm{H}), 2.33(\mathrm{q}, J=7.5 \mathrm{~Hz}, 2 \mathrm{H}), 2.28-2.18(\mathrm{~m}, 2 \mathrm{H}), 1.20-1.09(\mathrm{~m}, 4 \mathrm{H}), 1.14(\mathrm{t}, J=7.5 \mathrm{~Hz}, 3 \mathrm{H}), 0.69$ (t, $J$ $=6.9 \mathrm{~Hz}, 3 \mathrm{H}),-0.10(\mathrm{~s}, 9 \mathrm{H}) ;{ }^{13} \mathrm{C} \mathrm{NMR}\left(100 \mathrm{MHz}, \mathrm{CDCl}_{3}\right) \delta 146.5,136.4,135.7,135.2,128.6,127.9,106.8$, 96.9, 32.6, 31.6, 25.5, 22.6, 13.6, 13.6, -0.5; IR (neat) 3069, 3051, 2931, 2138, $2042 \mathrm{~cm}^{-1}$; HRMS (EI) m/z: $[\mathrm{M}]^{+}$Calcd for $\mathrm{C}_{25} \mathrm{H}_{34} \mathrm{GeSi}$ 436.1642; Found 436.1638.

(1) Li, Y.; Liu, X.; Jiang, H.; Feng, Z. Angew. Chem. Int. Ed. 2010, 49, 3338.

(2) (a) Hara, S.; Satoh, Y.; Ishiguro, H.; Suzuki, A. Tetrahedron Lett. 1983, 24, 735. (b) Martín, R.; Rivero, M. R.; Buchwald, S. L. Angew. Chem., Int. Ed. 2006, 45, 7079. 
The germanes 1c-1f and $\mathbf{3 a}-\mathbf{3 h}$ were synthesized from the corresponding bromides or iodides ${ }^{2,3}$ in the same manner as described for the preparation of $\mathbf{1 a}$.

(Z)-3-Ethyl-7-methyl-1-(trimethylsilyl)-4-(diphenylgermyl)-oct-3-en-1-yne (1c).<smiles>CCC(C#CCCCCCC(C)C)C(CCC(C)C)(c1ccccc1)c1ccccc1</smiles>

Colorless liquid (400 mg, $0.890 \mathrm{mmol}, 58 \%$ yield); ${ }^{1} \mathrm{H}$ NMR (300 MHz, $\left.\mathrm{CDCl}_{3}\right) \delta$ 7.55-7.50 (m, 4H), 7.36$7.20(\mathrm{~m}, 6 \mathrm{H}), 5.58$ (s, 1H), 2.32 (q, $J=7.5 \mathrm{~Hz}, 2 \mathrm{H}), 2.23-2.17$ (m, 2H), 1.29 (sept, $J=6.6 \mathrm{~Hz}, 1 \mathrm{H}), 1.17$ (t, $J=7.5 \mathrm{~Hz}, 3 \mathrm{H}), 1.09-1.01(\mathrm{~m}, 2 \mathrm{H}), 0.66(\mathrm{~d}, J=6.6 \mathrm{~Hz}, 6 \mathrm{H}),-0.10(\mathrm{~s}, 9 \mathrm{H}) ;{ }^{13} \mathrm{C} \mathrm{NMR}\left(100 \mathrm{MHz}, \mathrm{CDCl}_{3}\right) \delta$ 146.6, 136.4, 135.6, 135.3, 128.6, 127.9, 106.8, 96.8, 38.4, 30.8, 28.2, 25.4, 22.1, 13.6, -0.5; IR (neat) 3070, 3052, 2957, 2139, $2043 \mathrm{~cm}^{-1}$; HRMS (EI) m/z: [M] Calcd for $\mathrm{C}_{26} \mathrm{H}_{36} \mathrm{GeSi} 450.1798$; Found 450.1801.

(Z)-3-Ethyl-1-(trimethylsilyl)-4-cyclohexyl-4-(diphenylgermyl)-but-3-en-1-yne (1d).<smiles>CC#CC(=C(CC)CC)C1CCCCC1</smiles>

White solid (only pure fraction, $320 \mathrm{mg}, 0.694 \mathrm{mmol}, 32 \%$ yield); $\mathrm{Mp}=78.0-80.0{ }^{\circ} \mathrm{C} ;{ }^{1} \mathrm{H} \mathrm{NMR}(400 \mathrm{MHz}$, $\left.\mathrm{CDCl}_{3}\right) \delta$ 7.63-7.58 (m, 4H), 7.34-7.29 (m, 6H), $5.56(\mathrm{~s}, 1 \mathrm{H}), 2.75-2.67(\mathrm{~m}, 1 \mathrm{H}), 2.39$ (q, $\left.J=7.6 \mathrm{~Hz}, 2 \mathrm{H}\right)$, $1.75-1.67(\mathrm{~m}, 2 \mathrm{H}), 1.65-1.49(\mathrm{~m}, 4 \mathrm{H}), 1.32-1.22(\mathrm{~m}, 2 \mathrm{H}), 1.18-1.13(\mathrm{~m}, 1 \mathrm{H}), 1.15(\mathrm{t}, J=7.6 \mathrm{~Hz}, 3 \mathrm{H}), 1.12-$ $1.03(\mathrm{~m}, 1 \mathrm{H}),-0.22(\mathrm{~s}, 9 \mathrm{H}) ;{ }^{13} \mathrm{C} \mathrm{NMR}\left(100 \mathrm{MHz}, \mathrm{CDCl}_{3}\right) \delta 151.5,136.9,135.2,134.5,128.4,127.8,107.5$, 97.5, 42.8, 31.7, 26.5, 26.3, 25.8, 14.0, -0.7; IR (nujol) 3068, 2928, 2138, 2030, 1951, 1876, $1814 \mathrm{~cm}^{-1}$; HRMS (EI) m/z: [M] Calcd for $\mathrm{C}_{27} \mathrm{H}_{36} \mathrm{GeSi} 462.1798$; Found 462.1798 .

\section{1-[2-(Trimethylsilyl)ethynyl]-2-(diphenylgermyl)cyclohex-1-ene (1e).}<smiles>CC(C)C1CCCC1COc1ccccc1</smiles>

Colorless liquid (only pure fraction, $890 \mathrm{mg}, 2.20 \mathrm{mmol}, 54 \%$ yield); ${ }^{1} \mathrm{H}$ NMR (400 MHz, $\left.\mathrm{CDCl}_{3}\right) \delta 7.53-$ $7.51(\mathrm{~m}, 4 \mathrm{H}), 7.37-7.31(\mathrm{~m}, 6 \mathrm{H}), 5.62(\mathrm{~s}, 1 \mathrm{H}), 2.32-2.27(\mathrm{~m}, 2 \mathrm{H}), 2.17-2.13(\mathrm{~m}, 2 \mathrm{H}), 1.67-1.62(\mathrm{~m}, 2 \mathrm{H})$, $1.60-1.55(\mathrm{~m}, 2 \mathrm{H}),-0.01(\mathrm{~s}, 9 \mathrm{H}) ;{ }^{13} \mathrm{C}$ NMR $\left(100 \mathrm{MHz}, \mathrm{CDCl}_{3}\right) \delta$ 144.3, 135.8, 135.2, 131.1, 128.8, 128.1, 107.1, 95.5, 31.4, 31.2, 22.5, 22.2, -0.3; IR (neat) 3068, 2929, 2140, $2045 \mathrm{~cm}^{-1}$; HRMS (EI) m/z: [M] Calcd for $\mathrm{C}_{23} \mathrm{H}_{28} \mathrm{GeSi}$ 406.1172; Found 406.1171 .

(3) Kinoshita, H.; Fukumoto, H.; Ueda, A.; Miura, K. Tetrahedron 2018, 74, 1632. 
(Z)-3-Ethyl-1-(trimethylsilyl)-4-phenyl-4-(diphenylgermyl)-but-3-en-1-yne (1f).<smiles>CCC(C#CC(C)(C)C)=C(c1ccccc1)c1ccccc1</smiles>

Colorless liquid (only pure fraction, $381 \mathrm{mg}, 0.837 \mathrm{mmol}, 34 \%$ yield); ${ }^{1} \mathrm{H} \mathrm{NMR}\left(300 \mathrm{MHz}, \mathrm{CDCl}_{3}\right) \delta 7.45-$ $7.40(\mathrm{~m}, 4 \mathrm{H}), 7.34-7.23(\mathrm{~m}, 6 \mathrm{H}), 7.19-7.05(\mathrm{~m}, 3 \mathrm{H}), 6.86-6.81(\mathrm{~m}, 2 \mathrm{H}), 5.75(\mathrm{~s}, 1 \mathrm{H}), 2.18(\mathrm{q}, J=7.5 \mathrm{~Hz}$, 2H), $1.09(\mathrm{t}, J=7.5 \mathrm{~Hz}, 3 \mathrm{H}), 0.03(\mathrm{~s}, 9 \mathrm{H}) ;{ }^{13} \mathrm{C} \mathrm{NMR}\left(100 \mathrm{MHz}, \mathrm{CDCl}_{3}\right) \delta 147.2,141.7,137.3,135.9,135.1$, 128.7, 127.9, 127.9, 127.6, 125.9, 106.1, 99.2, 27.5, 13.6, -0.4; IR (neat) 3069, 3022, 2961, 2143, $2045 \mathrm{~cm}^{-}$ 1; HRMS (EI) m/z: [M] $]^{+}$Calcd for $\mathrm{C}_{27} \mathrm{H}_{30} \mathrm{GeSi}$ 456.1329; Found 456.1325.

\section{2-[2-(Trimethylsilyl)ethynyl]-1-(diphenylgermyl)benzene (3a).}<smiles>CC#Cc1ccccc1[Ge](c1ccccc1)(c1ccccc1)c1ccccc1</smiles>

Colorless liquid (995 mg, $2.48 \mathrm{mmol}, 62 \%$ yield); ${ }^{1} \mathrm{H}$ NMR (300 MHz, $\left.\mathrm{CDCl}_{3}\right) \delta$ 7.57-7.50 (m, 5H), 7.38$7.29(\mathrm{~m}, 9 \mathrm{H}), 5.89(\mathrm{~s}, 1 \mathrm{H}), 0.02(\mathrm{~s}, 9 \mathrm{H}) ;{ }^{13} \mathrm{C} \mathrm{NMR}\left(75 \mathrm{MHz}, \mathrm{CDCl}_{3}\right) \delta 139.0,135.7,135.4,135.3,132.4$, 129.5, 129.2, 129.0, 128.2, 128.1, 105.5, 97.7, -0.4; IR (neat) 3051, 2958, 2157, $2050 \mathrm{~cm}^{-1}$; HRMS (EI) $\mathrm{m} / \mathrm{z}:[\mathrm{M}]^{+}$Calcd for $\mathrm{C}_{23} \mathrm{H}_{24} \mathrm{GeSi}$ 402.0859; Found 402.0859 .

\section{5-Methyl-2-[2-(trimethylsilyl)ethynyl]-1-(diphenylgermyl)benzene (3b).}<smiles>CCC#Cc1ccc(C)cc1[Ge](c1ccccc1)(c1ccccc1)c1ccccc1</smiles>

Colorless liquid (522 mg, $1.26 \mathrm{mmol}, 63 \%$ yield); ${ }^{1} \mathrm{H}$ NMR $\left(300 \mathrm{MHz}, \mathrm{CDCl}_{3}\right) \delta 7.56-7.51(\mathrm{~m}, 4 \mathrm{H}), 7.45$ $(\mathrm{d}, J=7.8 \mathrm{~Hz}, 1 \mathrm{H}), 7.39-7.32(\mathrm{~m}, 6 \mathrm{H}), 7.17-7.12(\mathrm{~m}, 2 \mathrm{H}), 5.86(\mathrm{~s}, 1 \mathrm{H}), 2.27(\mathrm{~s}, 3 \mathrm{H}), 0.00(\mathrm{~s}, 9 \mathrm{H}) ;{ }^{13} \mathrm{C} \mathrm{NMR}$ $\left(75 \mathrm{MHz}_{2} \mathrm{CDCl}_{3}\right) \delta 138.7,138.1,136.3,135.5,135.3,132.3,130.0,128.9,128.1,126.3,105.7,96.8,21.5$, -0,4; IR (neat) 3069, 3049, 2958, 2156, $2049 \mathrm{~cm}^{-1}$; HRMS (EI) m/z: [M] Calcd for $\mathrm{C}_{24} \mathrm{H}_{26} \mathrm{GeSi}_{4}$ 416.1016; Found 416.1021.

\section{4,5-Dimethyl-2-[2-(trimethylsilyl)ethynyl]-1-(diphenylgermyl)benzene (3c).}<smiles>CCC#Cc1cc(C)c(OC)cc1[Ge](c1ccccc1)(c1ccccc1)c1ccccc1</smiles>

Colorless liquid (500 mg, $1.16 \mathrm{mmol}, 58 \%$ yield including some unidentified impurities, which could not be separated); ${ }^{1} \mathrm{H}$ NMR $\left(400 \mathrm{MHz}, \mathrm{CDCl}_{3}\right) \delta$ 7.55-7.52 (m, 4H), 7.41-7.32 (m, 7H), $7.12(\mathrm{~s}, 1 \mathrm{H}), 5.83(\mathrm{~s}$, 1H), 2.24 (s, 3H), 2.19 (s, 3H), 0.003 (s, 9H); $\left.{ }^{13} \mathrm{C} \mathrm{NMR} \mathrm{(100} \mathrm{MHz,} \mathrm{CDCl}_{3}\right) \delta 137.8,137.1,136.9,135.7$, 
135.3, 134.2, 133.6, 128.8, 128.1, 126.7, 105.8, 96.4, 19.7, 19.4, -0.4; IR (neat) 3069, 2958, 2150, 2047 $\mathrm{cm}^{-1}$; HRMS (EI) m/z: [M] $]^{+}$Calcd for $\mathrm{C}_{25} \mathrm{H}_{28} \mathrm{GeSi}$ 430.1172; Found 430.1180 .

\section{2-[2-(Trimethylsilyl)ethynyl]-3-(diphenylgermyl)naphthalene (3d).}<smiles>CC(C)C=Cc1ccccc1Oc1ccccc1</smiles>

Colorless gum (600 mg, $1.33 \mathrm{mmol}, 56 \%$ yield); ${ }^{1} \mathrm{H}$ NMR (300 MHz, $\left.\mathrm{CDCl}_{3}\right) \delta 8.08$ (s, 1H), $7.83(\mathrm{~s}, 1 \mathrm{H})$, 7.81-7.77 (m, 1H), 7,72-7.68 (m, 1H), 7.58-7.54 (m, 4H), 7.52-7.42 (m, 2H), 7.41-7.33 (m, 6H), 5.99 (s, $1 \mathrm{H}), 0.04$ (s, 9H); ${ }^{13} \mathrm{C}$ NMR (100 MHz, $\left.\mathrm{CDCl}_{3}\right) \delta 136.6,135.5,135.3,135.2,133.4,132.5,132.2,129.0$, 128.2, 127.9, 127.5, 127.1, 126.8, 125.5, 105.7, 97.6, -0.5; IR (neat) 3069, 3051, 2957, 2148, $2051 \mathrm{~cm}^{-1}$; HRMS (EI) m/z: [M] $]^{+}$Calcd for $\mathrm{C}_{27} \mathrm{H}_{26} \mathrm{GeSi}$ 452.1016; Found 452.1011 .

5-Fluoro-2-[2-(trimethylsilyl)ethynyl]-1-(diphenylgermyl)benzene (3e).

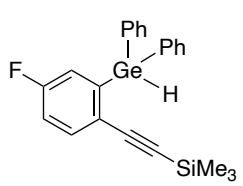

Colorless liquid (601 mg, $1.43 \mathrm{mmol}, 72 \%$ yield); ${ }^{1} \mathrm{H}$ NMR (300 MHz, $\left.\mathrm{CDCl}_{3}\right) \delta$ 7.56-7.49 (m, 5H), 7.41$7.33(\mathrm{~m}, 6 \mathrm{H}), 7.05-6.98(\mathrm{~m}, 2 \mathrm{H}), 5.90(\mathrm{~s}, 1 \mathrm{H}), 0.02(\mathrm{~s}, 9 \mathrm{H}) ;{ }^{13} \mathrm{C}$ NMR $\left(75 \mathrm{MHz}, \mathrm{CDCl}_{3}\right) \delta 162.2\left(\mathrm{~d},{ }^{1} J=\right.$ $252.8 \mathrm{~Hz}$ ), 142.4 (d, $\left.{ }^{3} J=4.5 \mathrm{~Hz}\right), 135.2,134.5,134.3$ (d, $\left.{ }^{3} J=7.5 \mathrm{~Hz}\right), 129.2,128.3,125.5,122.4\left(\mathrm{~d},{ }^{2} J=\right.$ $20.4 \mathrm{~Hz}$ ), 116.3 (d, ${ }^{2} J=22.6 \mathrm{~Hz}$ ), 104.5, 97.3, -0.5; IR (neat) 3069, 3052, 2959, 2158, $2057 \mathrm{~cm}^{-1}$; HRMS (EI) $\mathrm{m} / \mathrm{z}:[\mathrm{M}]^{+}$Calcd for $\mathrm{C}_{23} \mathrm{H}_{23} \mathrm{FGeSi} 420.0765$; Found 420.0760 .

\section{5-Chloro-2-[2-(trimethylsilyl)ethynyl]-1-(diphenylgermyl)benzene (3f).}

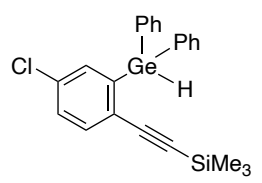

Colorless liquid (440 mg, $1.01 \mathrm{mmol}, 40 \%$ yield); ${ }^{1} \mathrm{H}$ NMR (300 MHz, $\left.\mathrm{CDCl}_{3}\right) \delta$ 7.53-7.49 (m, 4H), 7.49$7.44(\mathrm{~m}, 1 \mathrm{H}), 7.41-7.34(\mathrm{~m}, 6 \mathrm{H}), 7.33-7.28(\mathrm{~m}, 1 \mathrm{H}), 7.29(\mathrm{~s}, 1 \mathrm{H}), 5.88(\mathrm{~s}, 1 \mathrm{H}), 0.01(\mathrm{~s}, 9 \mathrm{H}) ;{ }^{13} \mathrm{C}$ NMR $(100$ $\left.\mathrm{MHz}, \mathrm{CDCl}_{3}\right) \delta 141.5$ 135.3, 135.2, 134.5, 134.1, 133.6, 129.3, 129.2, 128.5, 128.3, 104.3, 98.9, -0.5; IR (neat) 3070, 2960, 2157, $2057 \mathrm{~cm}^{-1}$; HRMS (EI) m/z: [M] Calcd for $\mathrm{C}_{23} \mathrm{H}_{23} \mathrm{ClGeSi}$ 436.0469; Found 436.0464 . 


\section{2-[2-(Diphenylmethylsilyl)ethynyl]-1-(diphenylgermyl)benzene (3g).}<smiles>C(=Cc1ccccc1Oc1ccccc1)c1ccccc1</smiles>

Colorless liquid (400 mg, $0.761 \mathrm{mmol}, 32 \%$ yield); ${ }^{1} \mathrm{H}$ NMR (400 MHz, $\left.\mathrm{CDCl}_{3}\right) \delta$ 7.64-7.62 (m, $\left.1 \mathrm{H}\right), 7.51$ 7.45 (m, 9H), 7.36-7.27 (m, 14H), $5.93(\mathrm{~s}, 1 \mathrm{H}), 0.54(\mathrm{~s}, 3 \mathrm{H}) ;{ }^{13} \mathrm{C} \mathrm{NMR}\left(100 \mathrm{MHz}, \mathrm{CDCl}_{3}\right) \delta 139.1,135.6$, 135.3, 135.1, 134.6, 134.2, 133.0, 129.5, 129.1, 129.0, 128.4, 128.4, 128.2, 127.8, 108.3, 93.7, -2.3; IR (neat) 3068, 3021, 2959, 2156, $2055 \mathrm{~cm}^{-1}$; HRMS (EI) m/z: [M] Calcd for $\mathrm{C}_{33} \mathrm{H}_{28} \mathrm{GeSi}$ 526.1172; Found 526.1172 .

\section{2-[2-(Triphenylsilyl)ethynyl]-1-(diphenylgermyl)benzene (3h).}

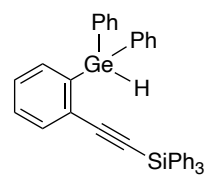

White solid (870 mg, $1.48 \mathrm{mmol}, 42 \%$ yield); $\mathrm{Mp}=120.0-121.0{ }^{\circ} \mathrm{C} ;{ }^{1} \mathrm{H}$ NMR $\left(400 \mathrm{MHz}, \mathrm{CDCl}_{3}\right) \delta 7.69$ $7.67(\mathrm{~m}, 1 \mathrm{H}), 7.52-7.49(\mathrm{~m}, 7 \mathrm{H}), 7.42-7.24(\mathrm{~m}, 21 \mathrm{H}), 5.94(\mathrm{~s}, 1 \mathrm{H}) ;{ }^{13} \mathrm{C} \mathrm{NMR}\left(100 \mathrm{MHz}, \mathrm{CDCl}_{3}\right) \delta 139.2$, 135.7, 135.6, 135.3, 135.0, 133.4, 133.3, 129.8, 129.1, 129.0, 128.6, 128.2, 127.8, 109.4, 92.3; IR (nujol) 3056, 2910, 2155, $2054 \mathrm{~cm}^{-1}$; HRMS (EI) m/z: [M] Calcd for $\mathrm{C}_{38} \mathrm{H}_{30} \mathrm{GeSi}$ 588.1329; Found 588.1335.

\section{2,5-Bis(diphenylgermyl)-1,4-bis[2-(trimethylsilyl)ethynyl]benzene (3i).}
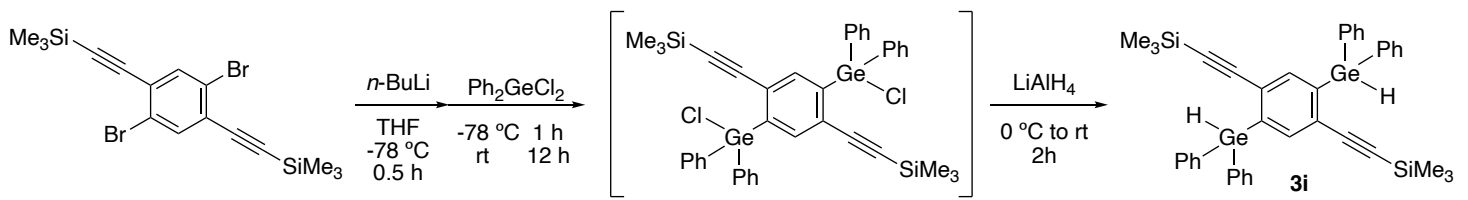

$n$ - $\mathrm{BuLi}$ (2.64 M in hexane, $1.40 \mathrm{~mL}, 3.69 \mathrm{mmol})$ was added to a THF solution of 1,4-dibromo-2,5-bis[2(trimethylsilyl)ethynyl]benzene $(720 \mathrm{mg}, 1.68 \mathrm{mmol})$ at $-78^{\circ} \mathrm{C}$. The mixture was stirred for $0.5 \mathrm{~h}$ at that temperature. Dichlorodiphenylgermane $(1.00 \mathrm{~g}, 3.69 \mathrm{mmol})$ was added to the mixture at $-78{ }^{\circ} \mathrm{C}$. The resultant mixture was stirred for $1 \mathrm{~h}$ at that temperature and then stirred at room temperature for $12 \mathrm{~h}$. $\mathrm{LiAlH}_{4}(140 \mathrm{mg}, 3.69 \mathrm{mmol})$ was added to the reaction mixture at $0{ }^{\circ} \mathrm{C}$. The mixture was stirred at room temperature for $2 \mathrm{~h}$. After quenching with water, the aqueous mixture was extracted with hexane $(30 \mathrm{~mL})$ three times. The combined organic layer was dried over $\mathrm{Na}_{2} \mathrm{SO}_{4}$ and concentrated. The residue was purified by silica-gel column chromatography (hexane/AcOEt $=20: 1$ ). The title compound was obtained as a white solid (648 mg, $0.895 \mathrm{mmol}, 53 \%$ yield). $\mathrm{Mp}=165.0-167.0{ }^{\circ} \mathrm{C} ;{ }^{1} \mathrm{H} \mathrm{NMR}\left(300 \mathrm{MHz}, \mathrm{CDCl}_{3}\right) \delta 7.56(\mathrm{~s}, 2 \mathrm{H})$, 7.54-7.50 (m, 8H), 7.40-7.32 (m, 12H), 5.86 (s, 2H), -0.03 (s, 18H); $\left.{ }^{13} \mathrm{C} \mathrm{NMR} \mathrm{(100} \mathrm{MHz,} \mathrm{CDCl}_{3}\right) \delta 140.2$, 139.1, 135.3, 134.8, 129.1, 128.8, 128.2, 105.2, 100.2, -0.6; IR (nujol) 3070, 3053, 2959, 2155, 2053863 $\mathrm{cm}^{-1}$; HRMS (EI) m/z: [M] Calcd for $\mathrm{C}_{40} \mathrm{H}_{42} \mathrm{Ge}_{2} \mathrm{Si}_{2}$ 726.1249; Found 726.1242 . 


\section{General Procedure for Synthesizing Germoles 2.}

1-Hydrogermyl-4-silyl-1,3-enyne $1(0.250 \mathrm{mmol})$, DIBAL-H (0.125 mL in $1.0 \mathrm{M}$ hexane solution, 0.125 $\mathrm{mmol})$, and octane $(0.75 \mathrm{~mL})$ were stirred in a two-necked flask at $80{ }^{\circ} \mathrm{C}$ for $1 \mathrm{~h}$. The reaction mixture was quenched with aqueous $1 \mathrm{M} \mathrm{HCl}(3 \mathrm{~mL})$ at room temperature, and the mixture was stirred for $1 \mathrm{~h}$. The aqueous mixture was extracted with hexane three times. The combined organic layer was dried over $\mathrm{Na}_{2} \mathrm{SO}_{4}$ and concentrated. The residue was purified by silica-gel column chromatography (hexane).

\section{1,1-Diphenyl-2,3-dipropyl-5-(trimethylsilyl)-1H-germole (2a).}

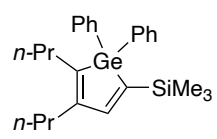

Colorless liquid (99.0 mg, $0.228 \mathrm{mmol}, 91 \%$ yield); ${ }^{1} \mathrm{H} \mathrm{NMR}\left(300 \mathrm{MHz}, \mathrm{CDCl}_{3}\right) \delta 7.50-7.54(\mathrm{~m}, 4 \mathrm{H}), 7.17-$ $7.37(\mathrm{~m}, 6 \mathrm{H}), 7.17(\mathrm{~s}, 1 \mathrm{H}), 2.30-2.40(\mathrm{~m}, 4 \mathrm{H}), 1.48-1.60(\mathrm{~m}, 2 \mathrm{H}), 1.18-1.31(\mathrm{~m}, 2 \mathrm{H}), 0.95(\mathrm{t}, J=7.2 \mathrm{~Hz}$, $3 \mathrm{H}), 0.69$ (t. $J=7.2 \mathrm{~Hz}, 3 \mathrm{H}),-0.07(\mathrm{~s}, 9 \mathrm{H}) ;{ }^{13} \mathrm{C} \mathrm{NMR}\left(125 \mathrm{MHz}, \mathrm{CDCl}_{3}\right) \delta 156.9,150.0,143.3,141.6$, 136.4, 134.8, 128.9, 128.2, 32.9, 32.4, 24.1, 22.3, 14.3, 14.0, -0.17; IR (neat) 3068, 2955, $\mathrm{cm}^{-1}$; HRMS (EI) $\mathrm{m} / \mathrm{z}:[\mathrm{M}]^{+}$Calcd for $\mathrm{C}_{25} \mathrm{H}_{34} \mathrm{GeSi}$ 436.1642; Found 436.1644 .

\section{1,1-Diphenyl-2-butyl-3-ethyl-5-(trimethylsilyl)-1 $H$-germole (2b).}

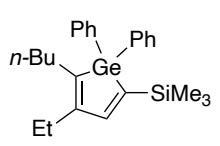

Colorless liquid (88.1 mg, $0.203 \mathrm{mmol}, 81 \%$ yield); ${ }^{1} \mathrm{H} \mathrm{NMR}\left(300 \mathrm{MHz}, \mathrm{CDCl}_{3}\right) \delta 7.54-7.50$ (m, 4H), 7.38$7.31(\mathrm{~m}, 6 \mathrm{H}), 7.20(\mathrm{~s}, 1 \mathrm{H}), 2.43-2.31(\mathrm{~m}, 4 \mathrm{H}), 1.23-1.15(\mathrm{~m}, 2 \mathrm{H}), 1.15-1.03(\mathrm{~m}, 2 \mathrm{H}), 1.11(\mathrm{t}, J=7.5 \mathrm{~Hz}$, $3 \mathrm{H}), 0.61(\mathrm{t}, J=7.2 \mathrm{~Hz}, 3 \mathrm{H}),-0.05(\mathrm{~s}, 9 \mathrm{H}) ;{ }^{13} \mathrm{C} \mathrm{NMR}\left(100 \mathrm{MHz}, \mathrm{CDCl}_{3}\right) \delta 156.6,151.6,142.6,141.7$, 136.4, 134.8, 128.9, 128.2, 32.8, 30.0, 23.5, 22.6, 13.9, 13.6, -0.2; IR (neat) 3069, 3052, 2957, $1710 \mathrm{~cm}^{-1}$; HRMS (EI) m/z: [M] $]^{+}$Calcd for $\mathrm{C}_{25} \mathrm{H}_{34} \mathrm{GeSi}$ 436.1642; Found 436.1648.

\section{1,1-Diphenyl-2-(3-methylbutyl)-3-ethyl-5-(trimethylsilyl)-1H-germole (2c).}<smiles>CCc1cc(C)c([P+]([O-])(c2ccccc2)c2ccccc2)c(CCC(C)C)c1C</smiles>

Colorless liquid (93.2 mg, $0.208 \mathrm{mmol}, 83 \%$ yield); ${ }^{1} \mathrm{H} \mathrm{NMR}\left(300 \mathrm{MHz}, \mathrm{CDCl}_{3}\right) \delta 7.54-7.49$ (m, 4H), 7.38$7.31(\mathrm{~m}, 6 \mathrm{H}), 7.20(\mathrm{~s}, 1 \mathrm{H}), 2.44-2.31(\mathrm{~m}, 4 \mathrm{H}), 1.33-1.25(\mathrm{~m}, 1 \mathrm{H}), 1.14-1.05(\mathrm{~m}, 2 \mathrm{H}), 1.11(\mathrm{t}, J=7.5 \mathrm{~Hz}$, $3 \mathrm{H}), 0.64(\mathrm{~d}, J=6.6 \mathrm{~Hz}, 6 \mathrm{H}),-0.05(\mathrm{~s}, 9 \mathrm{H}) ;{ }^{13} \mathrm{C} \mathrm{NMR}\left(100 \mathrm{MHz}, \mathrm{CDCl}_{3}\right) \delta 156.6,151.5,142.6,141.7$, 136.4, 134.8, 128.9, 128.2, 39.7, 28.0, 27.5, 23.5, 22.2, 13.9, -0.2; IR (neat) 3068, 3051, 2954, 2045, 1952, 1879, 1818, $833 \mathrm{~cm}^{-1}$; HRMS (EI) m/z: [M] Calcd for $\mathrm{C}_{26} \mathrm{H}_{36} \mathrm{GeSi} 450.1798$; Found 450.1790. 


\section{1,1-Diphenyl-2-cyclohexyl-3-ethyl-5-(trimethylsilyl)-1 $H$-germole (2d).}<smiles>CCC1=CC(C)CC1C1CCCCC1</smiles>

White solid (87.0 mg, $0.189 \mathrm{mmol}, 83 \%$ yield from $1 \mathrm{~g} 105 \mathrm{mg}, 0.227 \mathrm{mmol}$ ); $\mathrm{Mp}=44.0-45.0{ }^{\circ} \mathrm{C} ;{ }^{1} \mathrm{H} \mathrm{NMR}$ $\left(300 \mathrm{MHz}, \mathrm{CDCl}_{3}\right) \delta$ 7.61-7.57 (m, 4H), 7.39-7.32 (m, 6H), $7.16(\mathrm{~s}, 1 \mathrm{H}), 2.61-2.51(\mathrm{~m}, 1 \mathrm{H}), 2.37$ (q, $J=$ $7.5 \mathrm{~Hz}, 2 \mathrm{H}), 1.59-1.45$ (m, 5H), 1.25-1.09 (m, 2H), 1.14 (t, $J=7.5 \mathrm{~Hz}, 3 \mathrm{H}), 1.08-0.89$ (m, 3H), -0.13 (s, 9H); ${ }^{13} \mathrm{C} \mathrm{NMR}\left(100 \mathrm{MHz}, \mathrm{CDCl}_{3}\right) \delta 156.3,150.8,147.9,142.3,136.7,134.9,128.8,128.1,40.9,35.0,26.5$, 25.9, 23.9, 14.3, -0.3; IR (nujol) 3068, 2929, 1949, 1877, $1816 \mathrm{~cm}^{-1}$; HRMS (EI) m/z: [M] Calcd for $\mathrm{C}_{27} \mathrm{H}_{36} \mathrm{GeSi}$ 462.1798; Found 462.1799.

\section{1,1-Diphenyl-2-trimethylsilyl-4,5,6,7-tetrahydro-1H-benzo[b]germole (2e).}

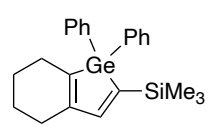

Pale yellow gum (80.0 mg, $0.197 \mathrm{mmol}, 79 \%$ yield); ${ }^{1} \mathrm{H}$ NMR (400 MHz, $\left.\mathrm{CDCl}_{3}\right) \delta$ 7.51-7.48 (m, 4H), 7.37-7.33 (m, 6H), 7.07 (s, 1H), 2.39-2.31 (m, 4H), 1.76-1.71 (m, 2H), 1.71-1.62 (m, 2H), -0.01 (s, 9H); ${ }^{13} \mathrm{C}$ NMR $\left(100 \mathrm{MHz}, \mathrm{CDCl}_{3}\right) \delta 156.4,148.6,141.4,140.0,136.1,134.8,129.0,128.2,28.4,27.2,23.4$, 22.9, -0.1; IR (neat) 3068, 2927, $838 \mathrm{~cm}^{-1}$; HRMS (EI) m/z: [M] Calcd for $\mathrm{C}_{23} \mathrm{H}_{28} \mathrm{GeSi}$ 406.1172; Found 406.1181 .

\section{1,1-Diphenyl-2-phenyl-3-ethyl-5-(trimethylsilyl)-1 $H$-germole (2f).}

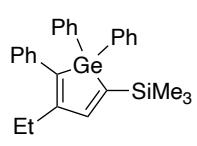

Colorless liquid (97.0 mg, $0.213 \mathrm{mmol}, 86 \%$ yield from $1 f 113 \mathrm{mg}, 0.248 \mathrm{mmol}$ ); ${ }^{1} \mathrm{H}$ NMR (400 MHz, $\left.\mathrm{CDCl}_{3}\right) \delta$ 7.49-7.46 (m, 4H), 7.38-7.29 (m, 7H), 7.21-7.15 (m, 2H), 7.12-7.07 (m, 1H), 7.04-7.00 (m, 2H), 2.49 (q, $J=7.6 \mathrm{~Hz}, 2 \mathrm{H}), 1.20$ (t, $J=7.6 \mathrm{~Hz}, 3 \mathrm{H}), 0.02(\mathrm{~s}, 9 \mathrm{H}) ;{ }^{13} \mathrm{C} \mathrm{NMR}\left(100 \mathrm{MHz}, \mathrm{CDCl}_{3}\right) \delta 156.3,152.4$, 144.6, 141.1, 140.9, 135.6, 134.9, 129.1, 128.3, 128.1, 128.0, 125.6, 24.3, 14.0, -0.2; IR (neat) 3068, 2955, 1953, 1879, $1819 \mathrm{~cm}^{-1}$; HRMS (EI) m/z: [M] $]^{+}$Calcd for $\mathrm{C}_{27} \mathrm{H}_{30} \mathrm{GeSi}$ 456.1329; Found 456.1336.

\section{Optyimaizarion of Reaction Conditions for Synthesis of Benzogermoles 4.}

We showed the full data of the optimized reaction conditions for synthesis of benzogermoles (Table SI-1). The reaction of 3a under the optimized conditions used for the synthesis of $\mathbf{2 a}$ gave the desired benzogermole $\mathbf{4 a}$ along with (E)- $\beta$-silyl styrene $\mathbf{5 a}$ (entry 1$)$. The reaction temperature is crucial to achieve the cyclization reaction. The reaction performed at $50^{\circ} \mathrm{C}$ for $2 \mathrm{~h}$ using 1.2 equiv of DIBAL-H gave 5a in $71 \%$ yield along with $\mathbf{3 a}$ (entry 3). Therefore, we performed the reaction at $80{ }^{\circ} \mathrm{C}$ to investigate the effect of reaction time (entries 2-7). The observation of $5 \mathbf{a}$ even after $12 \mathrm{~h}$ of reaction indicates that the cyclization of $3 \mathbf{a}$ to $4 \mathbf{a}$ is much slower than that of 1 to 2 (entry 6). Therefore, further extended reaction time was tested, and it was revealed that 3a was 
completely transformed into $\mathbf{4 a}$ after $24 \mathrm{~h}$ reaction without the formation of $\mathbf{5 a}$ (entry 7 ). The reduced amount of DIBAL-H used in the reaction improved the yield of $\mathbf{4 a}$ (entry 9).

Regarding the results of entry 1, after purification of the crude products by silica-gel column chromatography (hexane), we obtained 4a, 5a, and 3a as one fraction (total weight was $0.0904 \mathrm{~g}$ from $0.1003 \mathrm{~g}$ of $\mathbf{3 a}$ ) because of the almost same Rf values of $\mathbf{4 a}, \mathbf{5 a}$, and $\mathbf{3 a}$. We estimated the yields of $\mathbf{4 a}, \mathbf{5 a}$, and 3a based on the ${ }^{1} \mathrm{H}$ NMR: the integral values of trimethylsilyl group of them were $\mathbf{4 a}: \mathbf{5 a}: \mathbf{3 a}=4.26$ : $9.52: 3.88$. From these values and total weight of the mixture of them $(0.0904 \mathrm{~g})$, the yields of $4 \mathbf{a}, 5 \mathbf{a}$, and 3a were estimated $22 \%, 48 \%$, and $20 \%$, respectively. These yields of the products $\mathbf{4 a}$ and $\mathbf{5 a}$ also support that DIBAL-H re-generates after the germole formation and the DIBAL-H reacts with $\mathbf{3 a}$ again.

\section{Table SI-1. Optimization of Reaction Conditions for Synthesis of Benzogermoles ${ }^{a}$}

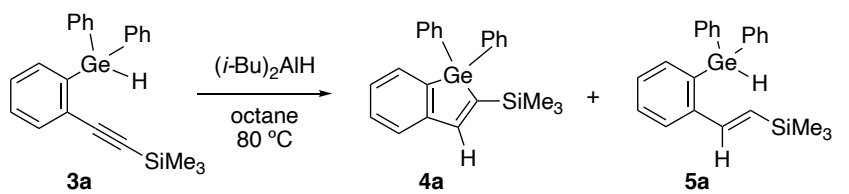

$\begin{array}{ccccc}\text { Entry } & \text { Time (h) } & \text { DIBAL-H (equiv) } & \text { Yield of } \mathbf{4} \mathbf{a}^{b}(\%) & {\text { Yield of } \mathbf{5} \mathbf{a}^{b}(\%)}^{(\%)} \\ 1 & 1 & 0.5 & 22 & 48^{c} \\ 2 & 1 & 1.2 & 28 & 42 \\ 3^{d} & 2 & 1.2 & 0 & 71^{e} \\ 4 & 6 & 1.2 & 88 & 6 \\ 5 & 8 & 1.2 & 84 & 2 \\ 6 & 12 & 1.2 & 85 & 1 \\ 7 & 24 & 1.2 & 82 & 0 \\ 8 & 6 & 0.5 & 33 & 17^{f} \\ 9 & 24 & 0.5 & 89 & 0\end{array}$

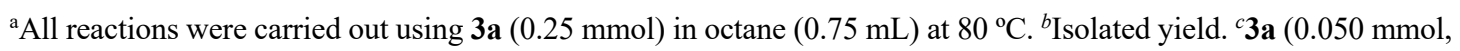
$20 \%$ ) was recovered. ${ }^{d}$ The reaction was conducted at $50{ }^{\circ} \mathrm{C} .{ }^{e} \mathbf{3 a}(0.069 \mathrm{mmol}, 28 \%)$ was recovered. ${ }_{\mathbf{3}} \mathbf{a}(0.048 \mathrm{mmol}$, $19 \%$ ) was recovered.

\section{General Procedure for Synthesizing Benzogermoles 4.}

1-Hydrogermyl-2-[2-(trimethylsilyl)ethynyl]benzene $3(0.250 \mathrm{mmol})$, DIBAL-H $(0.125 \mathrm{~mL}$ in $1.0 \mathrm{M}$ hexane solution, $0.125 \mathrm{mmol})$, and octane $(0.75 \mathrm{~mL})$ were stirred in a two-necked flask at $80{ }^{\circ} \mathrm{C}$ for $24 \mathrm{~h}$. The reaction mixture was quenched with aqueous $1 \mathrm{M} \mathrm{HCl}(3 \mathrm{~mL})$ at room temperature, and the mixture was stirred for $1 \mathrm{~h}$. The aqueous solution was extracted with hexane three times. The combined organic layer was dried over $\mathrm{Na}_{2} \mathrm{SO}_{4}$ and concentrated. The residue was purified by silica-gel column chromatography (hexane). 


\section{1,1-Diphenyl-2-trimethylsilyl-1 $H$-benzo[b]germole (4a).}<smiles>Cc1cc2ccccc2[R](-c2ccccc2)(-c2ccccc2)c1</smiles>

Colorless liquid (89.3 mg, $0.223 \mathrm{mmol}, 89 \%) ;{ }^{1} \mathrm{H}$ NMR (300 MHz, CDCl $) \delta 7.71(\mathrm{~d}, J=0.6 \mathrm{~Hz}, 1 \mathrm{H}), 7.60$ (dd, $J=6.9,0.9 \mathrm{~Hz}, 1 \mathrm{H}), 7.57-7.53(\mathrm{~m}, 4 \mathrm{H}), 7.41-7.31(\mathrm{~m}, 8 \mathrm{H}), 7.25$ (ddd, $J=6.6,6.6,2.4 \mathrm{~Hz}, 1 \mathrm{H}), 0.08$ (s, 9H); ${ }^{13} \mathrm{C} \mathrm{NMR}\left(75 \mathrm{MHz}, \mathrm{CDCl}_{3}\right) \delta 154.9,154.9,149.2,145.6,139.7,135.4,134.8,132.7,129.6,129.3$, 128.3, 127.8, 125.4, -0.1; IR (neat) 3052, 2952, 1955, 1882, $1817 \mathrm{~cm}^{-1}$; HRMS (EI) m/z: [M] Calcd for $\mathrm{C}_{23} \mathrm{H}_{24} \mathrm{GeSi}$ 402.0859; Found 402.0859.

(E)-1-(Diphenylgermyl)-2-(2-trimethylsilylethenyl)benzene (5a). A mixture with 3a $(5 a=71 \%, 3 a=$ $28 \%$, NMR yield) from 3a $100 \mathrm{mg}, 0.25 \mathrm{mmol})$.

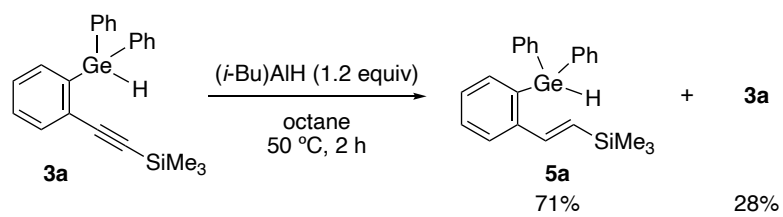

2-[2-(Trimethylsilyl)ethynyl]-1-(diphenylgermyl)benzene 3a (181 mg, $0.452 \mathrm{mmol})$, DIBAL-H (0.543 $\mathrm{mL}$ in $1.0 \mathrm{M}$ hexane solution, $0.543 \mathrm{mmol})$, and octane $(1.35 \mathrm{~mL})$ were stirred in a two-necked flask at 50 ${ }^{\circ} \mathrm{C}$ for $2 \mathrm{~h}$. The reaction mixture was quenched with aqueous $1 \mathrm{M} \mathrm{HCl}(6 \mathrm{~mL})$ at room temperature, and the mixture was stirred for $1 \mathrm{~h}$. The aqueous solution was extracted with hexane three times. The combined organic layer was dried over $\mathrm{Na}_{2} \mathrm{SO}_{4}$ and concentrated. The residue was purified by silica-gel column chromatography (hexane). The title compound 5a was obtained along with 3a without any other product ( $0.180 \mathrm{mg}$ as a mixture of $\mathbf{5 a}$ and $\mathbf{3 a},{ }^{1} \mathrm{H}$ NMR yield: $\mathbf{5 a} 71 \%$ yield and $\mathbf{3 a} 28 \%$ yield). Colorless liquid; ${ }^{1} \mathrm{H}$ $\operatorname{NMR}\left(400 \mathrm{MHz}, \mathrm{CDCl}_{3}\right) \delta 7.67(\mathrm{~d}, J=7.6 \mathrm{~Hz}, 1 \mathrm{H}), 7.56-7.49(\mathrm{~m}, 5 \mathrm{H}), 7.41-7.33(\mathrm{~m}, 7 \mathrm{H}), 7.21$ (ddd, $J=$ 7.6, 7.6, $0.8 \mathrm{~Hz}, 1 \mathrm{H}), 7.04(\mathrm{~d}, J=18.8 \mathrm{~Hz}, 1 \mathrm{H}), 6.35(\mathrm{~d}, J=18.8 \mathrm{~Hz}, 1 \mathrm{H}), 5.82(\mathrm{~s}, 1 \mathrm{H}),-0.07(\mathrm{~s}, 9 \mathrm{H}) ;{ }^{13} \mathrm{C}$ NMR (100 MHz, $\left.\mathrm{CDCl}_{3}\right) \delta 144.7,144.4,136.3,135.6,135.3,135.2,131.5,129.7,129.0,128.3,127.4$, 125.0, -1.5; IR (neat) 3068, 2955, 1432, $864 \mathrm{~cm}^{-1}$; HRMS (EI) m/z: [M] ${ }^{+}$Calcd for $\mathrm{C}_{23} \mathrm{H}_{26} \mathrm{GeSi}$ 404.1016; Found 404.1006 .

\section{6-Methyl-1,1-diphenyl-2-trimethylsilyl-1H-benzo[b]germole (4b).}

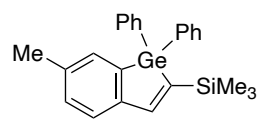

Colorless liquid (93.4 mg, $0.225 \mathrm{mmol}, 90 \%) ;{ }^{1} \mathrm{H}$ NMR (300 MHz, $\left.\mathrm{CDCl}_{3}\right) \delta 7.68(\mathrm{~d}, J=0.6 \mathrm{~Hz}, 1 \mathrm{H})$, 7.58-7.53 (m, 4H), 7.42-7.28 (m, 8H), 7.18-7.14 (m, 1H), $2.33(\mathrm{~s}, 3 \mathrm{H}), 0.07(\mathrm{~s}, 9 \mathrm{H}) ;{ }^{13} \mathrm{C}$ NMR $(75 \mathrm{MHz}$, $\left.\mathrm{CDCl}_{3}\right) \delta 154.8,146.7,143.9,139.9,137.6,135.6,134.8,133.7,130.2,129.2,128.3,125.1,21.4,-0.1$; IR (neat) 3067, 3049, 2953, $1248 \mathrm{~cm}^{-1}$; HRMS (EI) m/z: [M] ${ }^{+}$Calcd for $\mathrm{C}_{24} \mathrm{H}_{26} \mathrm{GeSi}$ 416.1016; Found 416.1015 . 
5,6-Dimethyl-1,1-diphenyl-2-trimethylsilyl-1H-benzo[b]germole (4c).<smiles></smiles>

Pale yellowish liquid (100 mg, $0.233 \mathrm{mmol}$, 93\% from 3c including some impurities. The yield was estimated based on the amount of $\mathbf{3 c}$ including some impurities considered as a pure sample.); ${ }^{1} \mathrm{H}$ NMR $\left(400 \mathrm{MHz}, \mathrm{CDCl}_{3}\right) \delta 7.67(\mathrm{~s}, 1 \mathrm{H}), 7.58-7.55(\mathrm{~m}, 4 \mathrm{H}), 7.39-7.32(\mathrm{~m}, 7 \mathrm{H}), 7.19(\mathrm{~s}, 1 \mathrm{H}), 2.29(\mathrm{~s}, 3 \mathrm{H}), 2.26(\mathrm{~s}$, $3 \mathrm{H}), 0.07(\mathrm{~s}, 9 \mathrm{H}) ;{ }^{13} \mathrm{C} \mathrm{NMR}\left(100 \mathrm{MHz}, \mathrm{CDCl}_{3}\right) \delta 154.8,147.5,144.0,137.8,136.6,136.2,135.8,134.8$, 134.2, 129.2, 128.3, 126.8, 20.0, 19.7, -0.1; IR (neat) 3068, 2954, $835 \mathrm{~cm}^{-1}$; HRMS (EI) m/z: [M] Calcd for $\mathrm{C}_{25} \mathrm{H}_{28} \mathrm{GeSi} 430.1172$; Found 430.1180 .

2-trimethylsilyl-1,1-diphenyl-1H-naphtho[2,3-b]germole (4d).<smiles>Cc1cc2cc3ccccc3cc2[R](-c2ccccc2)(-c2ccccc2)c1</smiles>

Colorless crystals (99.4 mg, $0.220 \mathrm{mmol}, 88 \%) ; \mathrm{Mp}=161.0-163.0{ }^{\circ} \mathrm{C} ;{ }^{1} \mathrm{H} \mathrm{NMR}\left(400 \mathrm{MHz}, \mathrm{CDCl}_{3}\right) \delta 8.04$ (s, 1H), $7.87(\mathrm{~s}, 1 \mathrm{H}), 7.84(\mathrm{~d}, J=7.6 \mathrm{~Hz}, 1 \mathrm{H}), 7.79-7.75(\mathrm{~m}, 2 \mathrm{H}), 7.61-7.57(\mathrm{~m}, 4 \mathrm{H}), 7.49-7.40(\mathrm{~m}, 2 \mathrm{H})$, 7.40-7.33 (m, 6H), $0.11(\mathrm{~s}, 9 \mathrm{H}) ;{ }^{13} \mathrm{C} \mathrm{NMR}\left(100 \mathrm{MHz}, \mathrm{CDCl}_{3}\right) \delta 155.0,147.3,146.4,137.5,135.6,134.8$, 134.6, 133.6, 133.3, 129.3, 128.4, 128.3, 128.1, 126.4, 126.0, 123.8, -0.2; IR (nujol) 3052, 2955, $838 \mathrm{~cm}^{-1}$; HRMS (EI) m/z: [M] $]^{+}$Calcd for $\mathrm{C}_{27} \mathrm{H}_{26} \mathrm{GeSi} 452.1016$; Found 452.1017.

\section{6-Fluoro-1,1-diphenyl-2-trimethylsilyl-1H-benzo[b]germole (4e).}<smiles>Cc1cc2ccc(F)cc2[R](-c2ccccc2)(-c2ccccc2)c1</smiles>

The obtained white solid after purification by silica-gel column chromatography (hexane) was dissolved in hexane and left to stand in the dark without vibration at room temperature. The crystals of $4 \mathbf{e}$ suitable for X-ray analysis were obtained by slow evaporation from hexane. Colorless crystals $(91.5 \mathrm{mg}, 0.218$ mmol, 91\% from 3e $100 \mathrm{mg}, 0.240 \mathrm{mmol}) ; \mathrm{Mp}=63.0-65.0{ }^{\circ} \mathrm{C} ;{ }^{1} \mathrm{H} \mathrm{NMR}\left(300 \mathrm{MHz}, \mathrm{CDCl}_{3}\right) \delta 7.67(\mathrm{~d}, J=$ $0.6 \mathrm{~Hz}, 1 \mathrm{H}), 7.56-7.51(\mathrm{~m}, 4 \mathrm{H}), 7.43-7.27(\mathrm{~m}, 8 \mathrm{H}), 7.06-6.99(\mathrm{~m}, 1 \mathrm{H}), 0.07(\mathrm{~s}, 9 \mathrm{H}) ;{ }^{13} \mathrm{C} \mathrm{NMR}(75 \mathrm{MHz}$, $\left.\mathrm{CDCl}_{3}\right) \delta 162.9\left(\mathrm{~d},{ }^{1} J=250.6 \mathrm{~Hz}\right), 153.8,145.2,145.2\left(\mathrm{~d},{ }^{3} \mathrm{~J}=5.4 \mathrm{~Hz}\right), 142.9\left(\mathrm{~d},{ }^{4} J=5.3 \mathrm{~Hz}\right), 134.8,134.7$, 129.5, 128.5, 126.2 (d, ${ }^{3} J=7.5 \mathrm{~Hz}$ ), 119.8 (d, ${ }^{2} J=20.4 \mathrm{~Hz}$ ), 116.1 (d, ${ }^{2} J=22.6 \mathrm{~Hz}$ ), -0.2; IR (nujol) 3070, 3048, 1580, 1526, $834 \mathrm{~cm}^{-1}$; HRMS (EI) m/z: [M] $]^{+}$Calcd for $\mathrm{C}_{23} \mathrm{H}_{23} \mathrm{FGeSi} 420.0765$; Found 420.0764.

6-Chloro-1,1-diphenyl-2-trimethylsilyl-1H-benzo[b]germole (4f).<smiles></smiles> 
Colorless liquid (58.3 mg, $0.134 \mathrm{mmol}, 54 \%) ;{ }^{1} \mathrm{H} \mathrm{NMR}\left(300 \mathrm{MHz}, \mathrm{CDCl}_{3}\right) \delta 7.66(\mathrm{~d}, J=0.9 \mathrm{~Hz}, 1 \mathrm{H})$, 7.54-7.50 (m, 5H), 7.41-7.30 (m, 8H), 0.07 (s, 9H); $\left.{ }^{13} \mathrm{C} \mathrm{NMR} \mathrm{(100} \mathrm{MHz,} \mathrm{CDCl}_{3}\right) \delta 153.7,147.4,146.5$, 142.4, 134.7, 134.6, 134.0, 132.6, 129.6, 129.5, 128.5, 126.1, -0.2; IR (neat) 3069, 3053, 2954, $835 \mathrm{~cm}^{-1}$; HRMS (EI) m/z: [M] ${ }^{+}$Calcd for $\mathrm{C}_{23} \mathrm{H}_{23} \mathrm{ClGeSi} 436.0469$; Found 436.0473.

\section{1,1-Diphenyl-2-methyl(diphenyl)silyl-1H-benzo[b]germole (4g).}

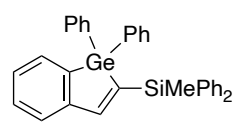

White solid (59.0 mg, $0.112 \mathrm{mmol}, 41 \%$ from $3 \mathrm{~g} 144 \mathrm{mg}, 0.274 \mathrm{mmol}) ; \mathrm{Mp}=112.0-114.0{ }^{\circ} \mathrm{C} ;{ }^{1} \mathrm{H}$ NMR $\left(400 \mathrm{MHz}, \mathrm{CDCl}_{3}\right) \delta 7.74$ (d, $\left.J=0.4 \mathrm{~Hz}, 1 \mathrm{H}\right), 7.62(\mathrm{dd}, J=6.8,0.8 \mathrm{~Hz}, 1 \mathrm{H}), 7.43-7.40(\mathrm{~m}, 4 \mathrm{H}), 7.37$ (d, $J$ $=4.0 \mathrm{~Hz}, 2 \mathrm{H}), 7.34-7.27(\mathrm{~m}, 9 \mathrm{H}), 7.24-7.18(\mathrm{~m}, 8 \mathrm{H}), 0.57(\mathrm{~s}, 3 \mathrm{H}) ;{ }^{13} \mathrm{C} \mathrm{NMR}\left(100 \mathrm{MHz}, \mathrm{CDCl}_{3}\right) \delta 158.6$, $149.0,141.5,139.9,136.5,135.1,135.0,134.7,134.0,132.9,129.6,129.1,129.1,128.2,127.6,125.8$, 2.3; IR (nujol) 3049, 2922, $944 \mathrm{~cm}^{-1}$; HRMS (EI) m/z: [M] Calcd for $\mathrm{C}_{33} \mathrm{H}_{28} \mathrm{GeSi}$ 526.1172; Found 526.1168 .

\section{1,1-Diphenyl-2-triphenylsilyl-1 $H$-benzo[b]germole (4h).}

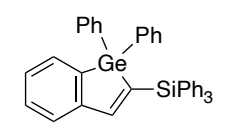

2-[2-(Triphenylsilyl)ethynyl]-1-(diphenylgermyl)benzene $\mathbf{3 h}$ was not soluble in octane. Therefore the reaction of $\mathbf{3 h}$ was conducted in 1,2-dichloroethane. $3 \mathbf{h}$ (148.7 mg, $0.250 \mathrm{mmol})$, DIBAL-H $(0.125 \mathrm{~mL}$ in $1.0 \mathrm{M}$ hexane solution, $0.125 \mathrm{mmol})$, and 1,2-dichloroethane $(0.75 \mathrm{~mL})$ were stirred in a two-necked flask at $80{ }^{\circ} \mathrm{C}$ for $24 \mathrm{~h}$. The reaction mixture was quenched with aqueous $1 \mathrm{M} \mathrm{HCl}(3 \mathrm{~mL})$ at room temperature, and the mixture was stirred for $1 \mathrm{~h}$. The aqueous solution was extracted with hexane three times. The combined organic layer was dried over $\mathrm{Na}_{2} \mathrm{SO}_{4}$ and concentrated. The residue was purified by silica-gel column chromatography (hexane/AcOEt $=10: 1$ ). The title compound $\mathbf{4 h}$ was obtained as colorless needle crystals (58.0 mg, $0.0987 \mathrm{mmol}, 39 \%) . \mathrm{Mp}=195.0-197.0{ }^{\circ} \mathrm{C} ;{ }^{1} \mathrm{H} \mathrm{NMR}\left(300 \mathrm{MHz}, \mathrm{CDCl}_{3}\right) \delta 7.90(\mathrm{~s}, 1 \mathrm{H})$, 7.64-7.62 (m, 1H), 7.44-7.27 (m, 13H), 7.25-7.10 (m, 15H); ${ }^{13} \mathrm{C} \mathrm{NMR}\left(100 \mathrm{MHz}, \mathrm{CDCl}_{3}\right) \delta 160.4,148.8$, $140.3,139.6,136.3,134.9,134.8,134.7,132.9,129.6,129.3,129.0,128.4,128.0,127.6,126.0$; IR (nujol) 3049, $2918822 \mathrm{~cm}^{-1}$; HRMS (EI) m/z: [M] $]^{+}$Calcd for $\mathrm{C}_{38} \mathrm{H}_{30} \mathrm{GeSi}$ 588.1329; Found 588.1322.

\section{1,5-Dihydro-1,1,5,5-tetraphenyl-2,6-bis(trimethylsilyl)-1,5-digerma-s-indacene (4i).}

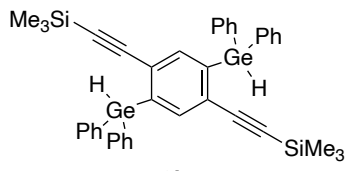

$3 \mathbf{i}$

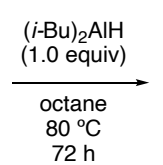

$72 \mathrm{~h}$

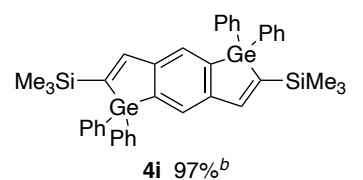

4i $97 \%{ }^{b}$

2,5-Bis(diphenylgermyl)-1,4-bis[2-(trimethylsilyl)ethynyl]benzene (3i) (181 mg, $0.250 \mathrm{mmol}$ ), DIBAL$\mathrm{H}(0.250 \mathrm{~mL}$ in $1.0 \mathrm{M}$ hexane solution, $0.250 \mathrm{mmol})$, and octane $(0.75 \mathrm{~mL})$ were stirred in a two-necked flask at $80{ }^{\circ} \mathrm{C}$ for $72 \mathrm{~h}$. The reaction mixture was quenched with aqueous $1 \mathrm{M} \mathrm{HCl}(6 \mathrm{~mL})$ at room 
temperature, and the mixture was stirred for $1 \mathrm{~h}$. The aqueous solution was extracted with hexane three times. The combined organic layer was dried over $\mathrm{Na}_{2} \mathrm{SO}_{4}$ and concentrated. The residue was purified by silica-gel column chromatography (hexane). The title compound was obtained as white solids (176 $\mathrm{mg}$, $0.243 \mathrm{mmol}, 97 \%) . \mathrm{Mp}=269.0-271.0{ }^{\circ} \mathrm{C} ;{ }^{1} \mathrm{H}$ NMR $\left(300 \mathrm{MHz}, \mathrm{CDCl}_{3}\right) \delta 7.69$ (s, 2H), 7.63 (s, 2H), 7.597.54 (m, 8H), 7.40-7.32 (m, 12H), 0.06 (s, $\left.18 \mathrm{H}) ;{ }^{13} \mathrm{C} \mathrm{NMR} \mathrm{(100} \mathrm{MHz,} \mathrm{CDCl}_{3}\right) \delta$ 155.1, 148.8, 146.0, 142.0, 135.4, 134.8, 129.6, 129.3, 128.3, -0.2; IR (nujol) 3051, 1532, $836 \mathrm{~cm}^{-1}$; HRMS (EI) m/z: [M] Calcd for $\mathrm{C}_{40} \mathrm{H}_{42} \mathrm{Ge}_{2} \mathrm{Si}_{2} 726.1249$; Found 726.1242 .

Triphenylgermylacetylene [4632-45-5].

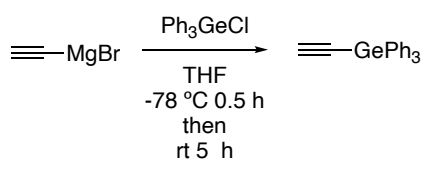

The title compound was synthesized from $\mathrm{Ph}_{3} \mathrm{GeCl}$ and ethynylmagnesium bromide. ${ }^{3,4}$ Colorless liquid

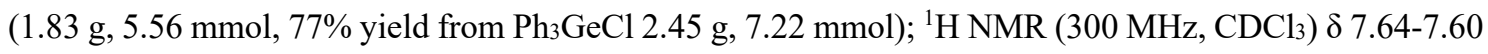
(m, 6H), 7.44-7.36 (m, 9H), $2.66(\mathrm{~s}, 1 \mathrm{H}) ;{ }^{13} \mathrm{C} \mathrm{NMR}\left(100 \mathrm{MHz}, \mathrm{CDCl}_{3}\right) \delta$ 134.5, 134.5, 129.6, 128.5, 95.9, 84.7; IR (neat) $3268,3069,2033 \mathrm{~cm}^{-1}$. The spectral properties of this compound were in satisfactory agreement with those previously reported.

\section{1-Bromo-2-[2-(triphenylgermyl)ethynyl]benzene.}

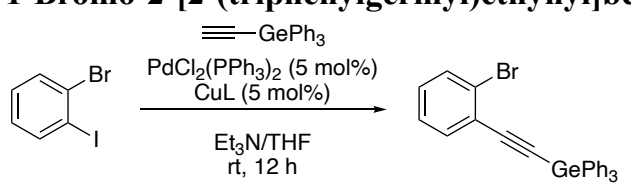

The title compound was synthesized from triphenylgermylacetylene and 2-bromoiodobenzene under the Sonogashira coupling conditions. ${ }^{3}$ White solid (1.59 g, $3.29 \mathrm{mmol}, 59 \%$ yield from 2-bromoiodobenzene $1.58 \mathrm{~g}, 5.57 \mathrm{mmol}) ; \mathrm{Mp}=90.0-91.0^{\circ} \mathrm{C} ;{ }^{1} \mathrm{H}$ NMR $\left(400 \mathrm{MHz}, \mathrm{CDCl}_{3}\right) \delta$ 7.74-7.69 (m, 6H), 7.62-7.58 (m, $2 \mathrm{H}), 7.45-7.38(\mathrm{~m}, 9 \mathrm{H}), 7.30-7.25(\mathrm{~m}, 1 \mathrm{H}), 7.19$ (ddd, $J=7.6,7.6,1.6 \mathrm{~Hz}, 1 \mathrm{H}) ;{ }^{13} \mathrm{C}$ NMR $(100 \mathrm{MHz}$, $\mathrm{CDCl}_{3}$ ) $\delta$ 135.0, 134.6, 134.0, 132.4, 129.7, 129.5, 128.4, 126.9, 126.0, 125.2, 105.9, 94.3; IR (nujol) 3049, 2925, $822 \mathrm{~cm}^{-1}$; HRMS (EI) m/z: [M] Calcd for $\mathrm{C}_{26} \mathrm{H}_{19} \mathrm{BrGe} 483.9882$; Found 483.9873.

\section{2-[2-(Trimethylgermyl)ethynyl]-1-(diphenylgermyl)benzene (6a).}

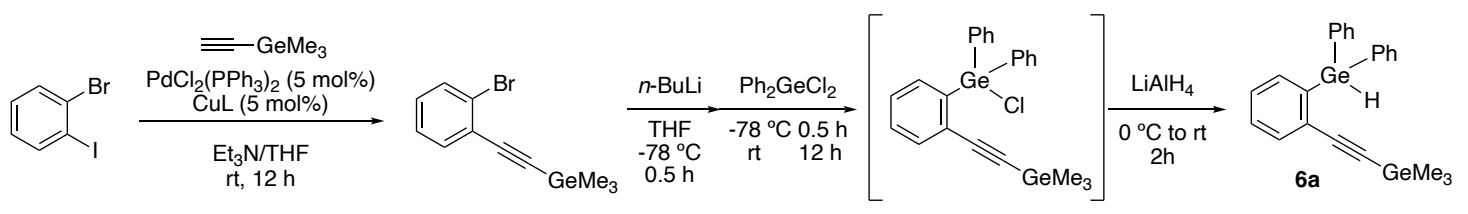

The title compound was prepared from dichlorodiphenylgermane and 1-bromo-2-[2(trimethylgermyl)ethynyl]benzene in the same manner as described for the preparation of 1a. 1-Bromo-2[2-(trimethylgermyl)ethynyl]benzene was prepared from trimethylgermylacetylene and 2bromoiodobenzene under the Sonogashira coupling conditions ${ }^{3}$ and used in the next step without

(4) David-Quillot, F.; Marsacq, D.; Balland, A.; Thibonnet, J.; Abarbri, M.; Duchêhe, A. Synthesis 2003, 3, 448. 
purification. Colorless liquid (only pure fraction, $341 \mathrm{mg}, 0.765 \mathrm{mmol}, 19 \%$ yield from 2bromoiodobenzene $1.14 \mathrm{~g}, 4.03 \mathrm{mmol}) ;{ }^{1} \mathrm{H}$ NMR $\left(300 \mathrm{MHz}, \mathrm{CDCl}_{3}\right) \delta$ 7.55-7.51 (m, 5H), 7.39-7.30 (m, $8 \mathrm{H}), 7.27-7.21(\mathrm{~m}, 1 \mathrm{H}), 5.88(\mathrm{~s}, 1 \mathrm{H}), 0.19(\mathrm{~s}, 9 \mathrm{H}) ;{ }^{13} \mathrm{C} \mathrm{NMR}\left(100 \mathrm{MHz}, \mathrm{CDCl}_{3}\right) \delta 138.8,135.6,135.5$, 135.3, 132.2, 129.8, 129.1, 128.9, 128.2, 127.8, 104.7, 98.4, -0.6; IR (neat) 3069, 3051, 2979, 2154, 2050, $834 \mathrm{~cm}^{-1}$; HRMS (EI) m/z: [M] Calcd for $\mathrm{C}_{23} \mathrm{H}_{24} \mathrm{Ge}_{2}$ 448.0302; Found 448.0308 .

\section{2-[2-(Triphenylgermyl)ethynyl]-1-(diphenylgermyl)benzene (6b).}

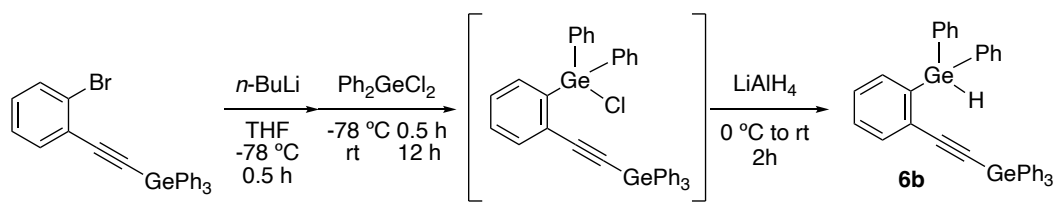

The title compound was prepared from dichlorodiphenylgermane and 1-bromo-2-[2(triphenylgermyl)ethynyl]benzene in the same manner as described for the preparation of 1a. White solid (only pure fraction, $221 \mathrm{mg}, \quad 0.350 \mathrm{mmol}, \quad 0.22 \%$ yield from 1-bromo-2-[2(triphenylgermyl)ethynyl]benzene $769 \mathrm{mg}, 1.59 \mathrm{mmol}$ ); $\mathrm{Mp}=98.0-99.0{ }^{\circ} \mathrm{C} ;{ }^{1} \mathrm{H} \mathrm{NMR}\left(300 \mathrm{MHz}, \mathrm{CDCl}_{3}\right)$ $\delta$ 7.69-7.65 (m, 1H), 7.49-7.22 (m, 28H), $5.95(\mathrm{~s}, 1 \mathrm{H}) ;{ }^{13} \mathrm{C}$ NMR (100 MHz, $\left.\mathrm{CDCl}_{3}\right) \delta$ 139.0, 135.5, 135.3, 135.1, 135.0, 134.6, 133.1, 129.4, 129.3, 129.1, 128.9, 128.3, 128.2, 108.0, 92.1; IR (nujol) 3049, 2924, 2153, 2063, $817 \mathrm{~cm}^{-1}$; HRMS (EI) m/z: [M] $]^{+}$Calcd for $\mathrm{C}_{38} \mathrm{H}_{30} \mathrm{Ge}_{2}$ 634.0771; Found 634.0771.

\section{General Procedure for Synthesizing 2-Germylbenzogermoles 7.}

Germylacetylene derivative $6(0.250 \mathrm{mmol})$, DIBAL-H $(0.125 \mathrm{~mL}$ in $1.0 \mathrm{M}$ hexane solution, 0.125 $\mathrm{mmol})$, and octane $(0.75 \mathrm{~mL})$ were stirred in a two-necked flask at $80{ }^{\circ} \mathrm{C}$ for $24 \mathrm{~h}$. The reaction mixture was quenched with aqueous $1 \mathrm{M} \mathrm{HCl}(3 \mathrm{~mL})$ at room temperature, and the mixture was stirred for $1 \mathrm{~h}$. The aqueous solution was extracted with hexane three times. The combined organic layer was dried over $\mathrm{Na}_{2} \mathrm{SO}_{4}$ and concentrated. The residue was purified by silica-gel column chromatography (hexane).

\section{1,1-Diphenyl-2-trimethylgermyl-1 $H$-benzo[b]germole (7a).}

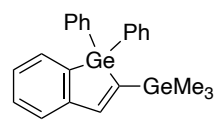

Colorless liquid (96.9 mg, $0.218 \mathrm{mmol}, 87 \%$ yield); ${ }^{1} \mathrm{H} \mathrm{NMR}\left(400 \mathrm{MHz}, \mathrm{CDCl}_{3}\right) \delta 7.62(\mathrm{~d}, J=0.4 \mathrm{~Hz}, 1 \mathrm{H})$, $7.60(\mathrm{dd}, J=6.8,0.8 \mathrm{~Hz}, 1 \mathrm{H}), 7.56-7.53(\mathrm{~m}, 4 \mathrm{H}), 7.40-7.32(\mathrm{~m}, 8 \mathrm{H}), 7.25-7.21(\mathrm{~m}, 1 \mathrm{H}), 0.21(\mathrm{~s}, 9 \mathrm{H}) ;{ }^{13} \mathrm{C}$ NMR $\left(100 \mathrm{MHz}, \mathrm{CDCl}_{3}\right) \delta 153.3,149.1,147.7,139.4,135.4,134.7,132.7,129.6,129.3,128.3,127.4$, 125.0, -0.8; IR (neat) 3052, 2970, $823 \mathrm{~cm}^{-1}$; HRMS (EI) m/z: [M] Calcd for $\mathrm{C}_{23} \mathrm{H}_{24} \mathrm{Ge}_{2}$ 448.0302; Found 448.0303 .

\section{1,1-Diphenyl-2-triphenylgermyl-1 $H$-benzo[b]germole (7b).}

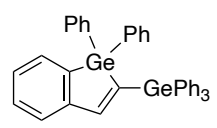


2-[2-(Triphenylgermyl)ethynyl]-1-(diphenylgermyl)benzene $\mathbf{6} \mathbf{b}$ was not soluble in octane. Therefore the reaction of $\mathbf{6 b}$ was conducted in 1,2-dichloroethane. $\mathbf{6 b}(158.0 \mathrm{mg}, 0.250 \mathrm{mmol})$, DIBAL-H $(0.125 \mathrm{~mL}$ in $1.0 \mathrm{M}$ hexane solution, $0.125 \mathrm{mmol})$, and 1,2-dichloroethane $(0.75 \mathrm{~mL})$ were stirred in a two-necked flask at $80{ }^{\circ} \mathrm{C}$ for $24 \mathrm{~h}$. The reaction mixture was quenched with aqueous $1 \mathrm{M} \mathrm{HCl}(3 \mathrm{~mL})$ at room temperature, and the mixture was stirred for $1 \mathrm{~h}$. The aqueous solution was extracted with hexane three times. The combined organic layer was dried over $\mathrm{Na}_{2} \mathrm{SO}_{4}$ and concentrated. The residue was purified by silica-gel column chromatography (hexane/AcOEt $=10: 1$ ). The title compound $\mathbf{7 b}$ was obtained as a white solid (51.3 mg, $0.0812 \mathrm{mmol}, 32 \%$ yield). $\mathrm{Mp}=184.0-186.0{ }^{\circ} \mathrm{C} ;{ }^{1} \mathrm{H}$ NMR $\left(400 \mathrm{MHz}, \mathrm{CDCl}_{3}\right) \delta 7.81$ (d, $J=0.4$ $\mathrm{Hz}, 1 \mathrm{H}), 7.64(\mathrm{dd}, J=6.8,0.8 \mathrm{~Hz}, 1 \mathrm{H}), 7.40-7.36(\mathrm{~m}, 8 \mathrm{H}), 7.34-7.12(\mathrm{~m}, 20 \mathrm{H}) ;{ }^{13} \mathrm{C}$ NMR $(100 \mathrm{MHz}$, $\left.\mathrm{CDCl}_{3}\right) \delta 158.1,148.7,141.5,139.7,136.7,135.3,134.7,132.9,129.6,129.1,128.8,128.1,128.0,125.6$; IR (nujol) 3049, 2923, $812 \mathrm{~cm}^{-1}$; HRMS (EI) m/z: [M] $]^{+}$Calcd for $\mathrm{C}_{38} \mathrm{H}_{30} \mathrm{Ge}_{2}$ 634.0771; Found 634.0768.

\section{2-Iodo-1,1-diphenyl-1 $H$-benzo[b]germole (8).}

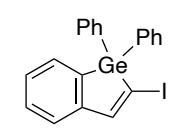

1,1-Diphenyl-2-trimethylsilyl-1H-benzo[b]germole (4a, $201 \mathrm{mg}, 0.500 \mathrm{mmol})$ and $N$-iodosuccininide (NIS, $112 \mathrm{mg}, 0.500 \mathrm{mmol}$ ) were stirred in $\mathrm{CH}_{2} \mathrm{Cl}_{2}(5 \mathrm{~mL})$ at room temperature for $6 \mathrm{~h}$. Saturated aqueous $\mathrm{Na}_{2} \mathrm{~S}_{2} \mathrm{O}_{3}(3 \mathrm{~mL})$ was added to the reaction mixture, and the resultant aqueous mixture was stirred for 15 $\min$. The aqueous mixture was extracted with hexane/AcOEt (10:1) three times. The combined organic layer was dried over $\mathrm{Na}_{2} \mathrm{SO}_{4}$ and concentrated. The residue was purified by silica-gel column chromatography (hexane/AcOEt $=30: 1$ ). 2-Iodo-1,1-diphenyl-1 $H$-benzo[b]germole $(\mathbf{8})$ was obtained as white solids (45.5 mg, $0.100 \mathrm{mmol}, 20 \%$ yield). $\mathrm{Mp}=149.0-150.0^{\circ} \mathrm{C} ;{ }^{1} \mathrm{H} \mathrm{NMR}\left(400 \mathrm{MHz}, \mathrm{CDCl}_{3}\right) \delta 7.95$ (s, 1H), $7.64(\mathrm{~d}, J=6.9 \mathrm{~Hz}, 1 \mathrm{H}), 7.62-7.58(\mathrm{~m}, 4 \mathrm{H}), 7.47-7.36(\mathrm{~m}, 6 \mathrm{H}), 7.36-7.24(\mathrm{~m}, 3 \mathrm{H}) ;{ }^{13} \mathrm{C}$ NMR $(100$ $\mathrm{MHz}, \mathrm{CDCl}_{3}$ ) $\delta 154.7,149.5,137.3,134.7,133.0,132.9,130.0,130.0,128.7,127.6,124.6,96.6$; IR (nujol) 3056, 3004, $884 \mathrm{~cm}^{-1}$; HRMS (EI) m/z: [M] $]^{+}$Calcd for $\mathrm{C}_{20} \mathrm{H}_{15} \mathrm{GeI} 455.9430$; Found 455.9433. 


\section{X-ray Crystallographic Data of 4e (CCDC 2075032)}
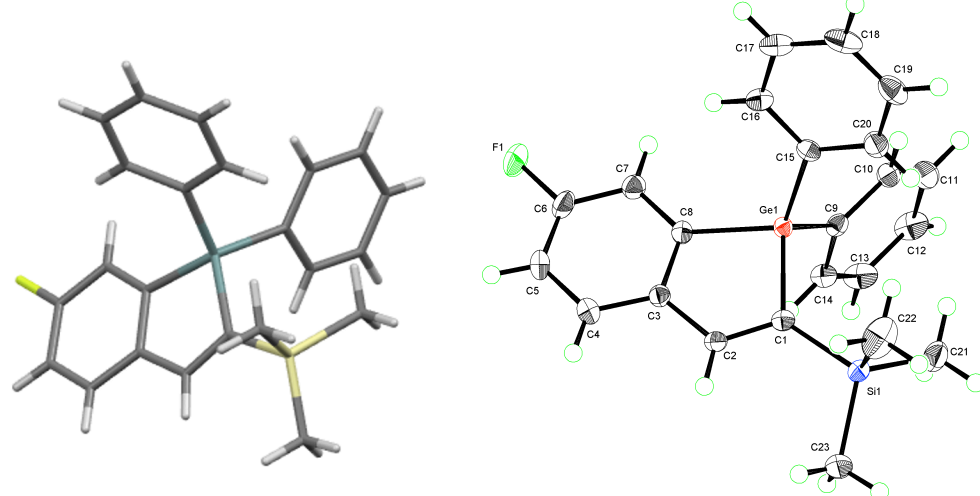

Capped sticks model of $4 \mathbf{e}$ (left) and ORTEP drawing of $4 \mathbf{e}$ (thermal ellipsoids at $50 \%$ probability) (right).

Table S1. Crystal data and structure refinement for $4 \mathrm{e}$.

\begin{tabular}{|c|c|}
\hline Identification code & 4e (CCDC 2075032) \\
\hline Empirical formula & $\mathrm{C}_{23} \mathrm{H}_{23} \mathrm{FGeSi}$ \\
\hline Formula weight & 419.09 \\
\hline Temperature & $100(2) \mathrm{K}$ \\
\hline Wavelength & $0.71073 \AA$ \\
\hline Crystal system & monoclinic \\
\hline Space group & $\mathrm{P} 2{ }_{1} / \mathrm{n}$ \\
\hline \multirow[t]{3}{*}{ Unit cell dimensions } & $\mathrm{a}=8.8092(9) \AA$ \\
\hline & $\beta=99.3110(10)^{\circ}$. \\
\hline & $\mathrm{c}=12.1177(12) \AA$ \\
\hline Volume & $2132.6(4) \AA^{3}$ \\
\hline $\mathrm{Z}$ & 4 \\
\hline Density (calculated) & $1.305 \mathrm{Mg} / \mathrm{m}^{3}$ \\
\hline Absorption coefficient & $1.504 \mathrm{~mm}^{-1}$ \\
\hline $\mathrm{F}(000)$ & 864 \\
\hline Crystal size & $0.27 \times 0.21 \times 0.05 \mathrm{~mm}^{3}$ \\
\hline Theta range for data collection & 1.98 to $27.50^{\circ}$. \\
\hline Index ranges & $-11<=\mathrm{h}<=11,-26<=\mathrm{k}<=26,-15<=\mathrm{l}<=15$ \\
\hline Reflections collected & 23953 \\
\hline Independent reflections & $4875[\mathrm{R}($ int $)=0.1744]$ \\
\hline Completeness to theta $=27.50^{\circ}$ & $99.5 \%$ \\
\hline Max. and min. transmission & 0.9286 and 0.6868 \\
\hline Refinement method & Full-matrix least-squares on $\mathrm{F}^{2}$ \\
\hline Data / restraints / parameters & $4875 / 0 / 238$ \\
\hline Goodness-of-fit on $\mathrm{F}^{2}$ & 1.061 \\
\hline Final R indices $[\mathrm{I}>2 \operatorname{sigma}(\mathrm{I})]$ & $\mathrm{R} 1=0.0393, \mathrm{wR} 2=0.1067$ \\
\hline $\mathrm{R}$ indices (all data) & $\mathrm{R} 1=0.0426, \mathrm{wR} 2=0.1088$ \\
\hline Largest diff. peak and hole & 0.887 and -1.213 e. $\AA^{-3}$ \\
\hline
\end{tabular}




\section{NMR Spectra}

(Z)-1-(Trimethylsilyl)-4-(diphenylgermyl)-3-propylhept-3-en-1-yne (1a).

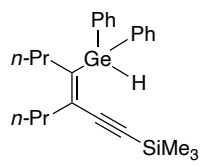

${ }^{1} \mathrm{H} \mathrm{NMR}\left(300.13 \mathrm{MHz}, \mathrm{CDCl}_{3}, \mathrm{CHCl}_{3}: \delta 7.26 \mathrm{ppm}\right),{ }^{13} \mathrm{C} \mathrm{NMR}\left(75.47 \mathrm{MHz}, \mathrm{CDCl}_{3}: \delta 77.0 \mathrm{ppm}\right)$
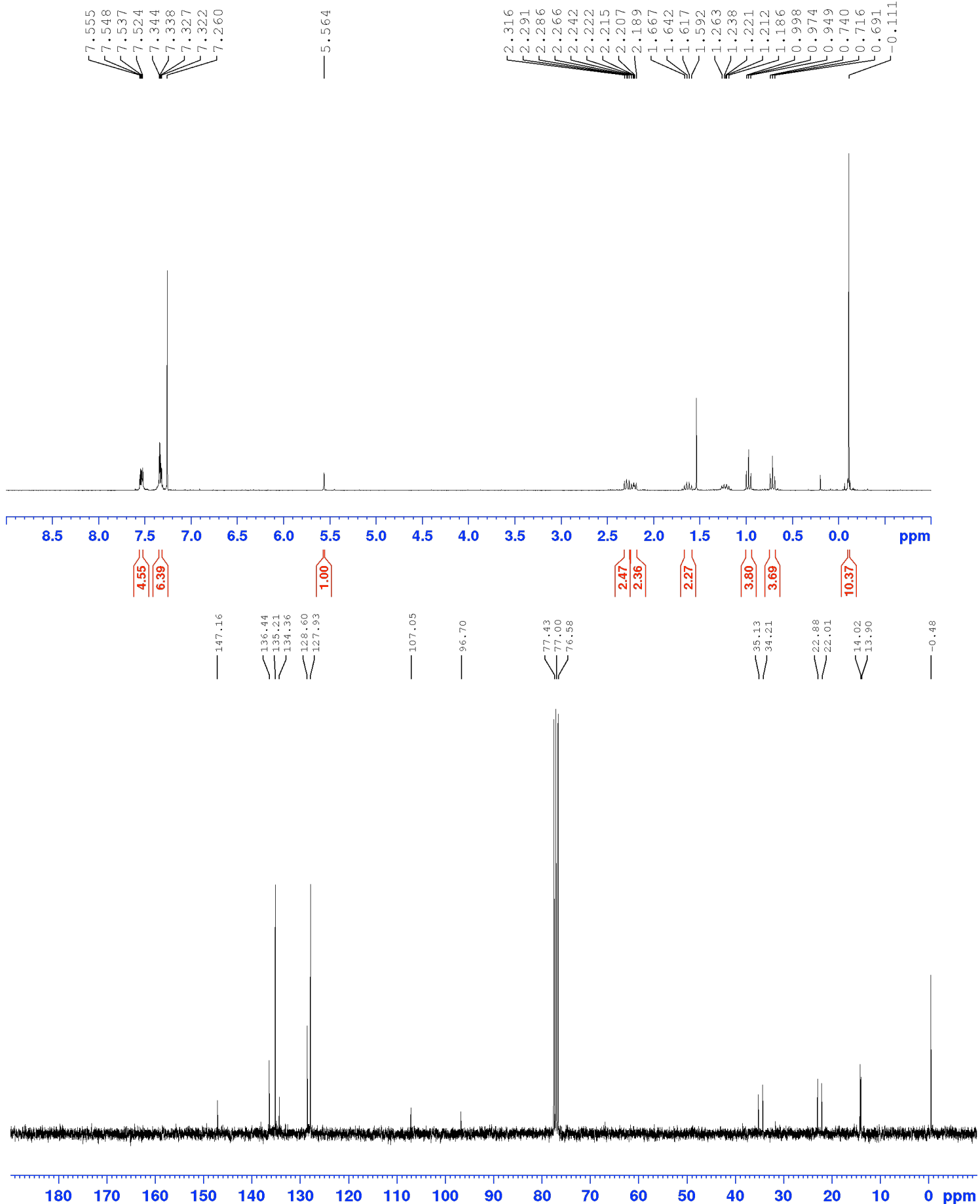
(Z)-3-Ethyl-1-(trimethylsilyl)-4-(diphenylgermyl)-oct-3-en-1-yne (1b).

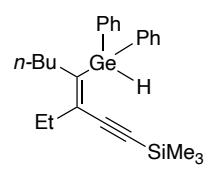

${ }^{1} \mathrm{H}$ NMR (300.13 MHz, $\left.\mathrm{CDCl}_{3}, \mathrm{CHCl}_{3}: \delta 7.26 \mathrm{ppm}\right),{ }^{13} \mathrm{C} \mathrm{NMR}\left(100.61 \mathrm{MHz}, \mathrm{CDCl}_{3}: \delta 77.0 \mathrm{ppm}\right)$
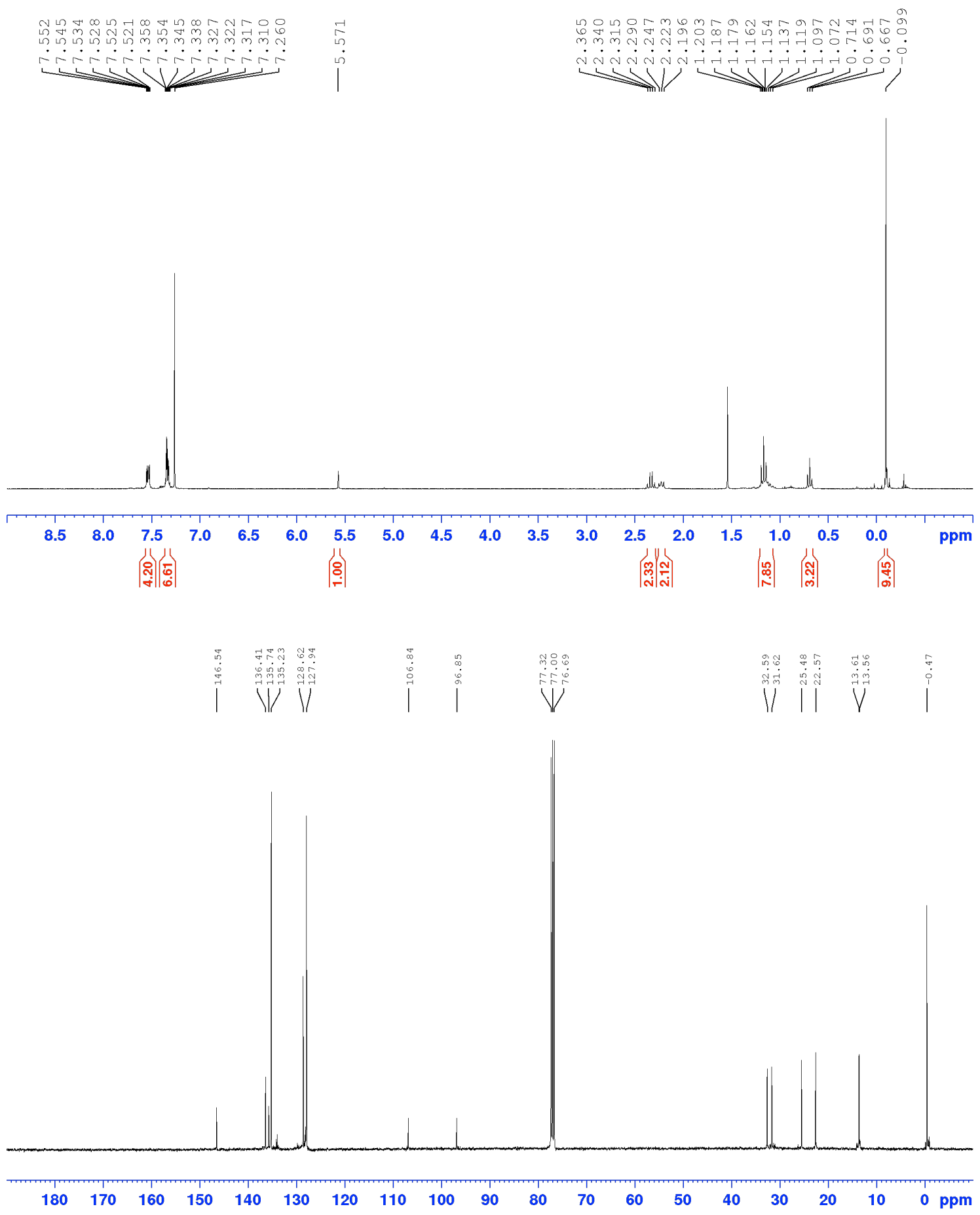
(Z)-3-Ethyl-7-methyl-1-(trimethylsilyl)-4-(diphenylgermyl)-oct-3-en-1-yne (1c).<smiles>CCC(C#C[As])=C(CCC(C)C)[Ge](c1ccccc1)(c1ccccc1)c1ccccc1</smiles>

${ }^{1} \mathrm{H}$ NMR (300.13 MHz, $\left.\mathrm{CDCl}_{3}, \mathrm{CHCl}_{3}: \delta 7.26 \mathrm{ppm}\right),{ }^{13} \mathrm{C} \mathrm{NMR}\left(100.61 \mathrm{MHz}, \mathrm{CDCl}_{3}: \delta 77.0 \mathrm{ppm}\right)$

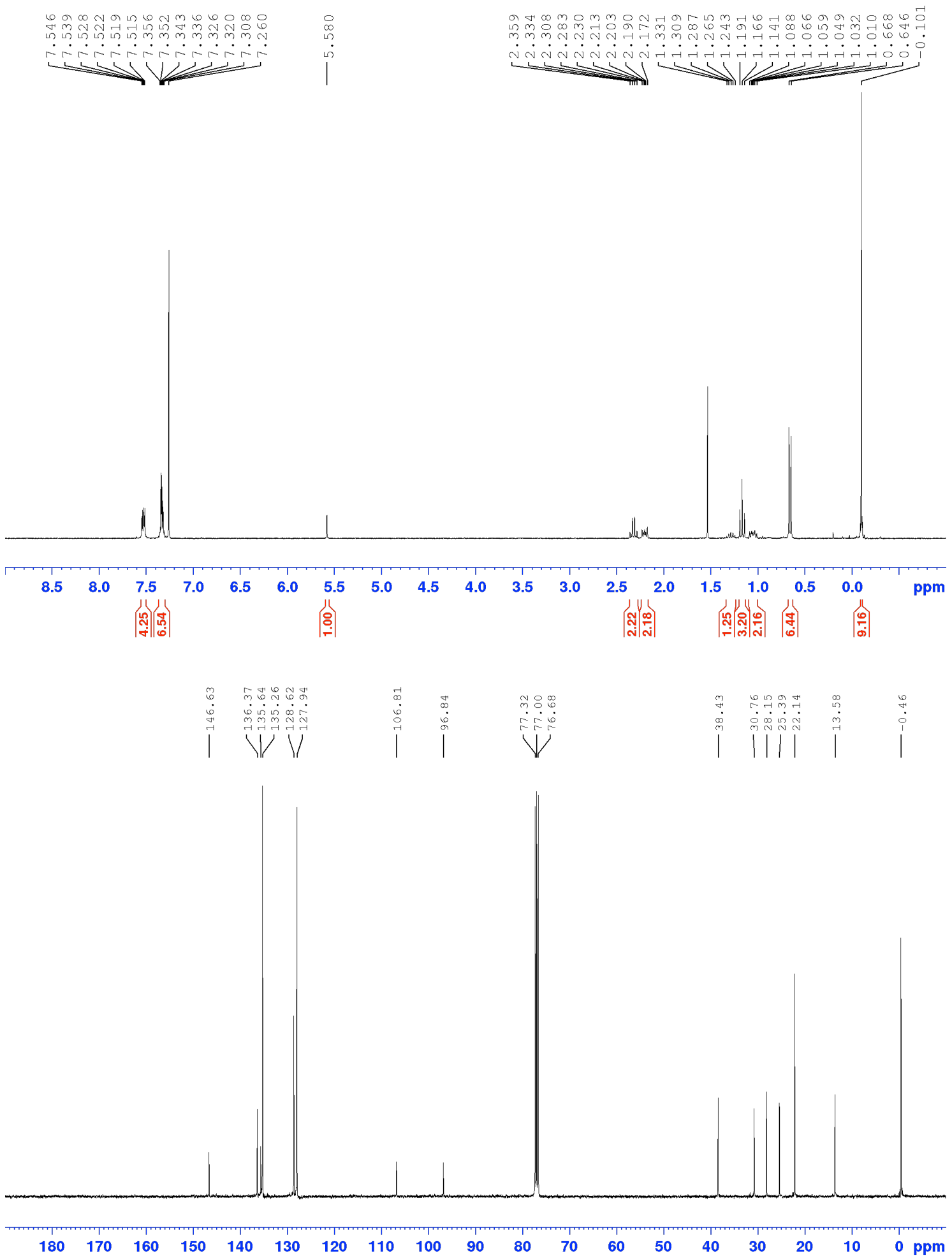


(Z)-3-Ethyl-1-(trimethylsilyl)-4-cyclohexyl-4-(diphenylgermyl)-but-3-en-1-yne (1d).<smiles>CC#C/C(CC)=C(/C1CCCCC1)C(c1ccccc1)(c1ccccc1)c1ccccc1</smiles>

${ }^{1} \mathrm{H}$ NMR (400.13 MHz, $\left.\mathrm{CDCl}_{3}, \mathrm{CHCl}_{3}: \delta 7.26 \mathrm{ppm}\right),{ }^{13} \mathrm{C} \mathrm{NMR}\left(100.61 \mathrm{MHz}, \mathrm{CDCl}_{3}: \delta 77.0 \mathrm{ppm}\right)$
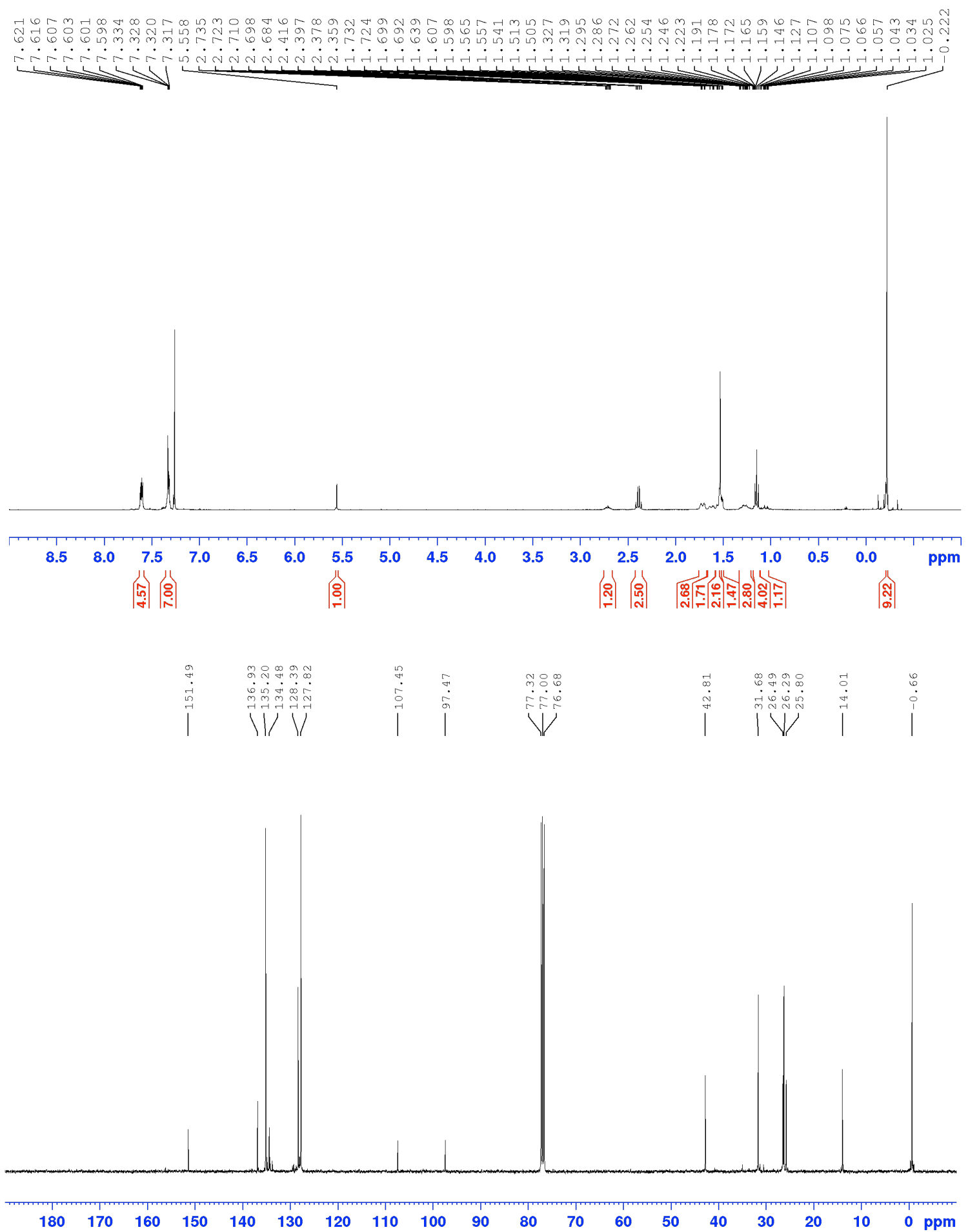
1-[2-(Trimethylsilyl)ethynyl]-2-(diphenylgermyl)cyclohex-1-ene (1e).<smiles>CC=CC1CCCCC1CCC</smiles>

${ }^{1} \mathrm{H} \mathrm{NMR}\left(400.13 \mathrm{MHz}, \mathrm{CDCl}_{3}, \mathrm{CHCl}_{3}: \delta 7.26 \mathrm{ppm}\right),{ }^{13} \mathrm{C} \mathrm{NMR}\left(100.61 \mathrm{MHz}, \mathrm{CDCl}_{3}: \delta 77.0 \mathrm{ppm}\right)$
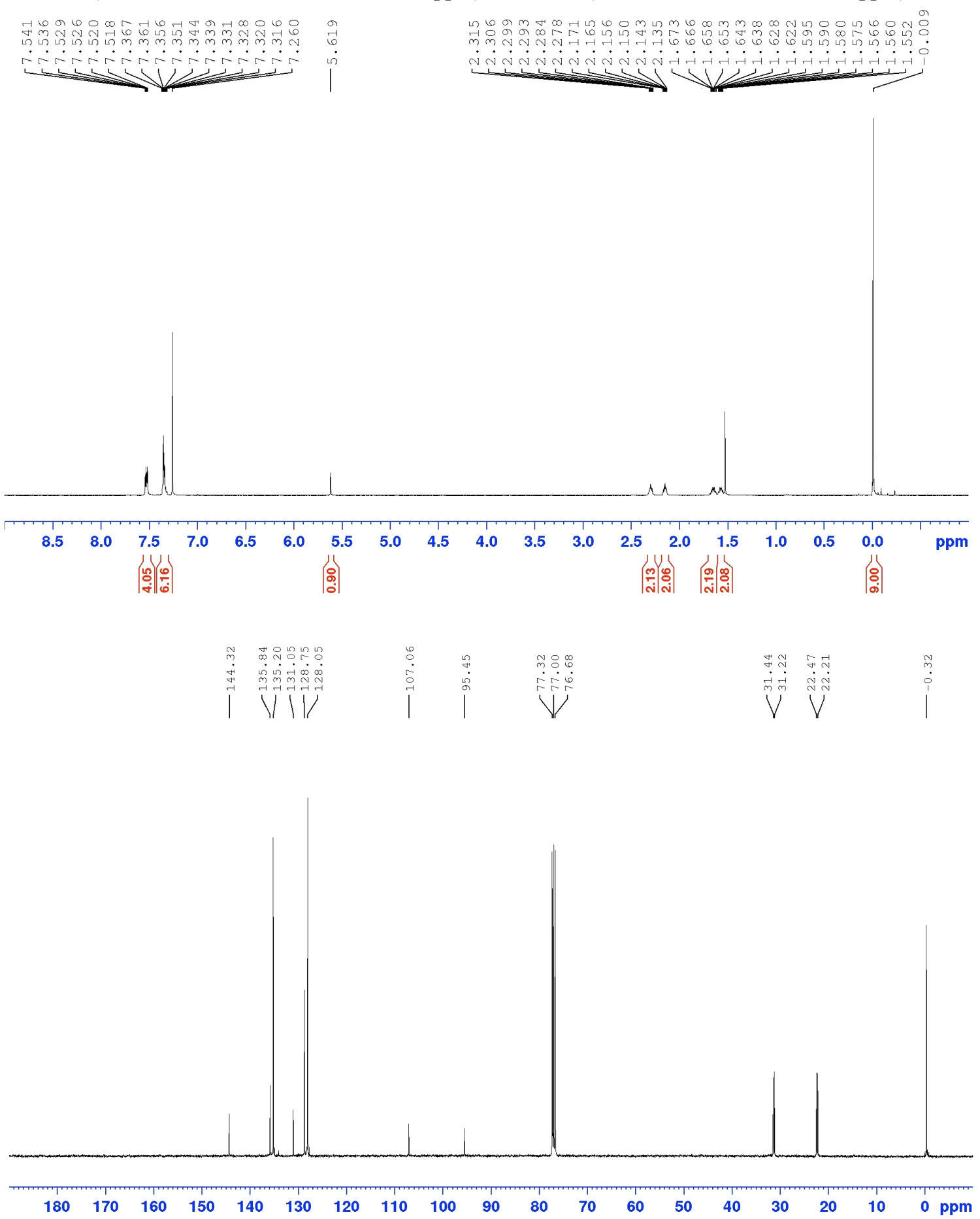
(Z)-3-Ethyl-1-(trimethylsilyl)-4-phenyl-4-(diphenylgermyl)-but-3-en-1-yne (1f).

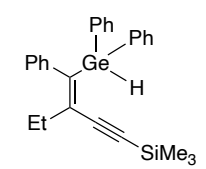

${ }^{1} \mathrm{H}$ NMR (300.13 MHz, $\left.\mathrm{CDCl}_{3}, \mathrm{CHCl}_{3}: \delta 7.26 \mathrm{ppm}\right),{ }^{13} \mathrm{C} \mathrm{NMR}\left(100.61 \mathrm{MHz}, \mathrm{CDCl}_{3}: \delta 77.0 \mathrm{ppm}\right)$
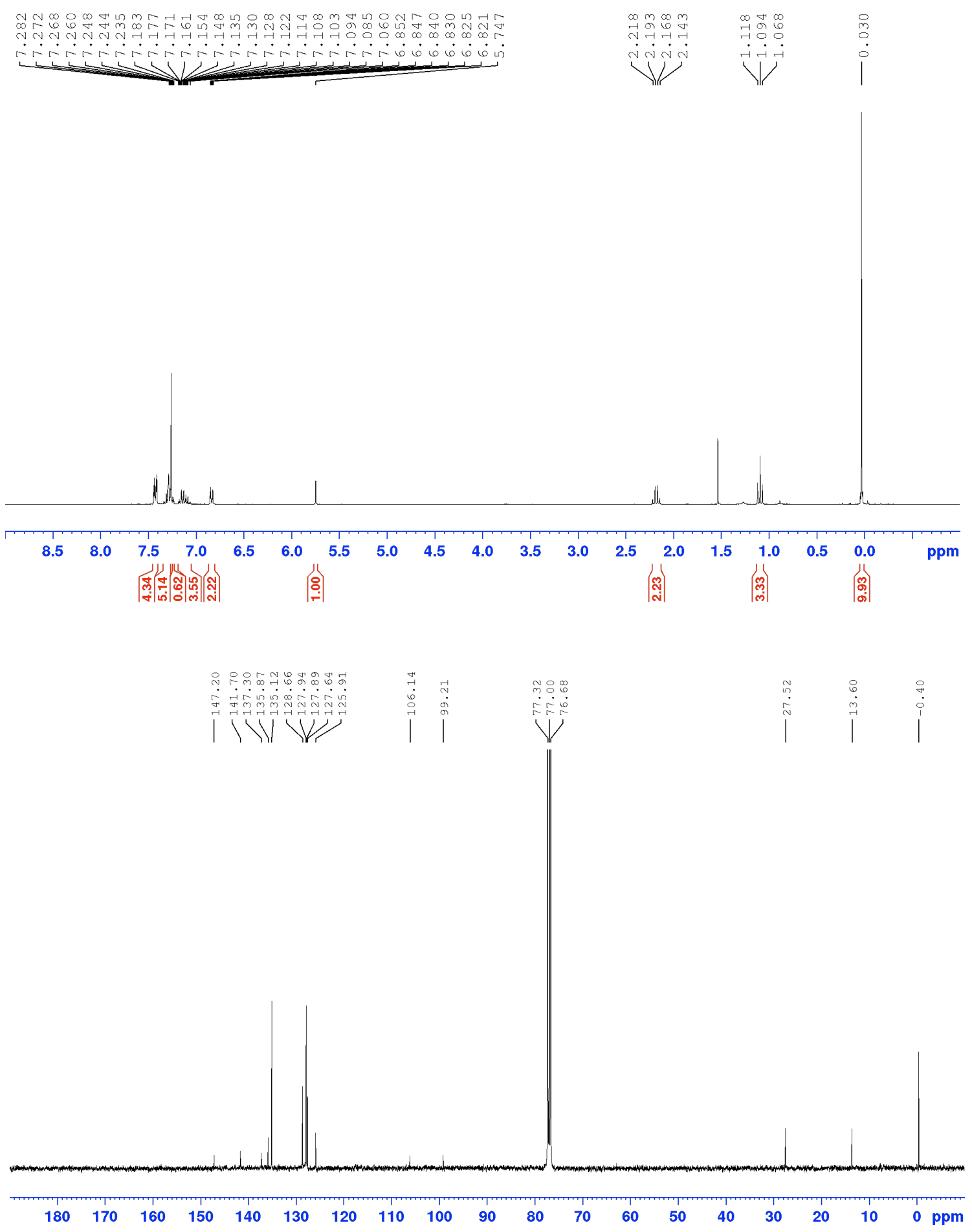
2-[2-(Trimethylsilyl)ethynyl]-1-(diphenylgermyl)benzene (3a).<smiles>[Mg]C=Cc1ccccc1Oc1ccccc1</smiles>

${ }^{1} \mathrm{H} \mathrm{NMR}\left(300.13 \mathrm{MHz}, \mathrm{CDCl}_{3}, \mathrm{CHCl}_{3}: \delta 7.26 \mathrm{ppm}\right),{ }^{13} \mathrm{C} \mathrm{NMR}\left(75.47 \mathrm{MHz}, \mathrm{CDCl}_{3}: \delta 77.0 \mathrm{ppm}\right)$
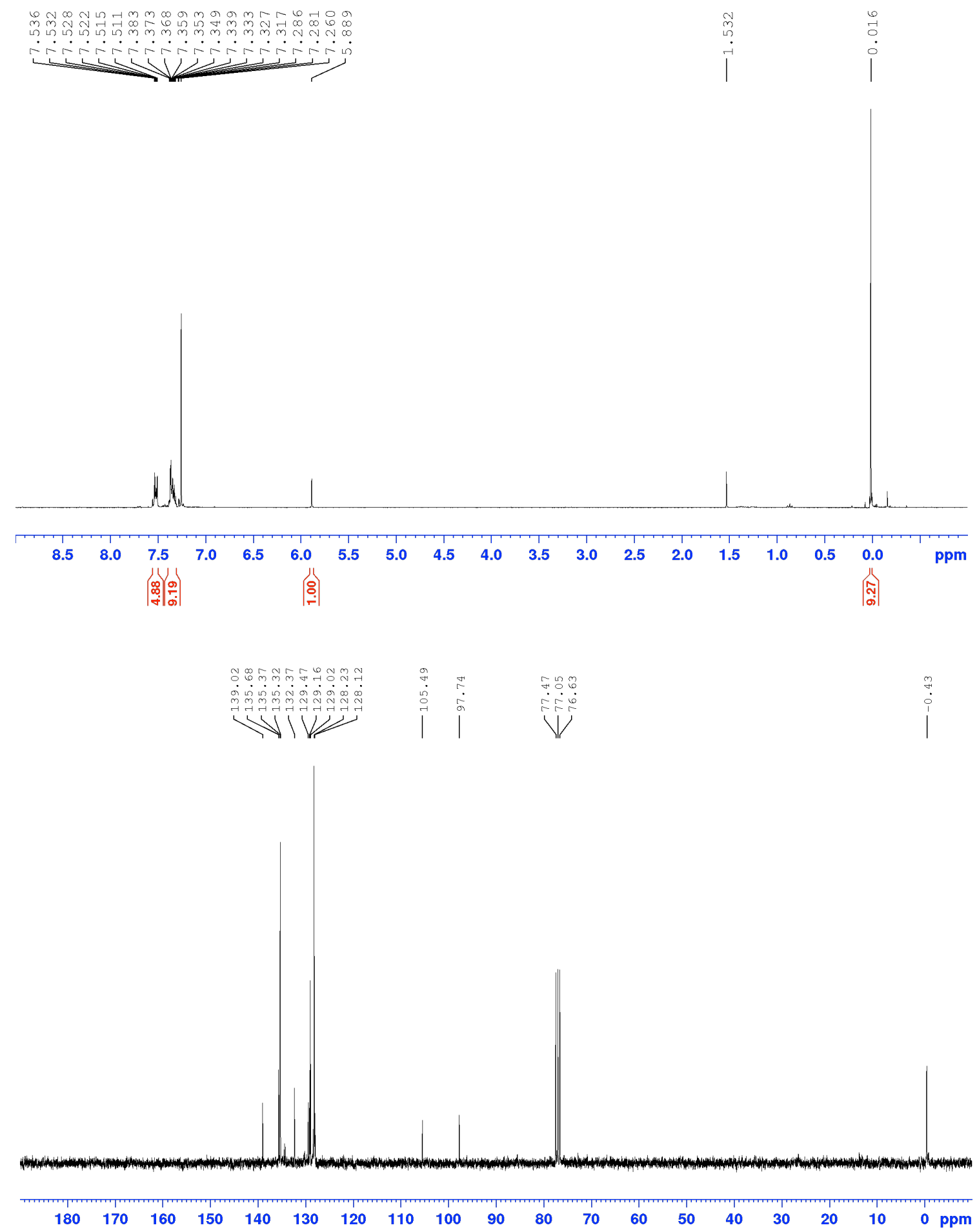
5-Methyl-2-[2-(trimethylsilyl)ethynyl]-1-(diphenylgermyl)benzene (3b).<smiles>CC#Cc1ccc(C)cc1Oc1ccccc1</smiles>

${ }^{1} \mathrm{H}$ NMR (300.13 MHz, $\left.\mathrm{CDCl}_{3}, \mathrm{CHCl}_{3}: \delta 7.26 \mathrm{ppm}\right),{ }^{13} \mathrm{C} \mathrm{NMR}\left(75.47 \mathrm{MHz}, \mathrm{CDCl}_{3}: \delta 77.0 \mathrm{ppm}\right)$
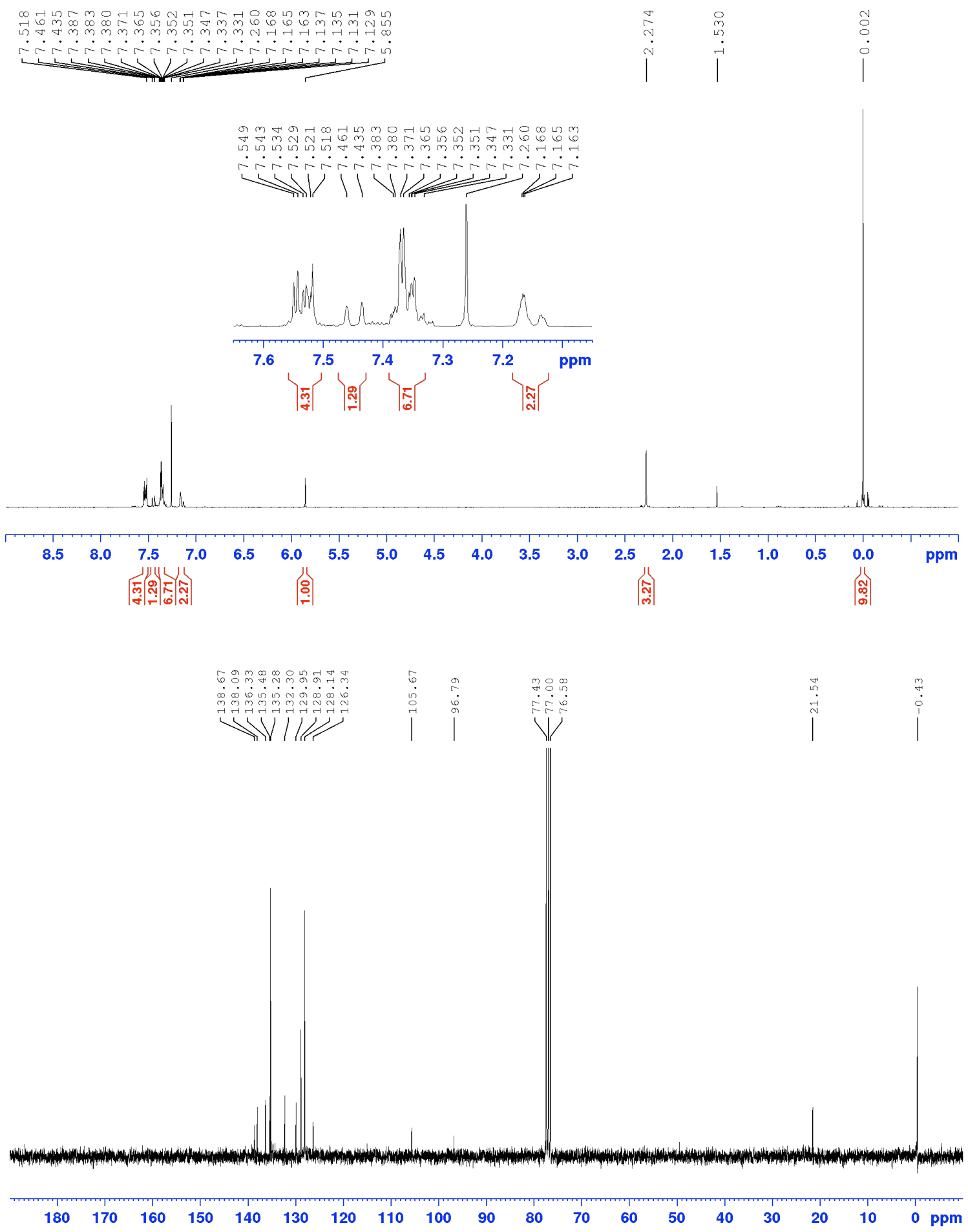
4,5-Dimethyl-2-[2-(trimethylsilyl)ethynyl]-1-(diphenylgermyl)benzene (3c).

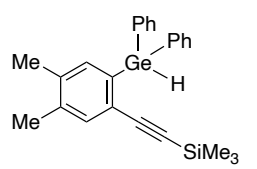

${ }^{1} \mathrm{H} \mathrm{NMR}\left(400.13 \mathrm{MHz}, \mathrm{CDCl}_{3}, \mathrm{CHCl}_{3}: \delta 7.26 \mathrm{ppm}\right),{ }^{13} \mathrm{C} \mathrm{NMR}\left(100.61 \mathrm{MHz}, \mathrm{CDCl}_{3}: \delta 77.0 \mathrm{ppm}\right)$
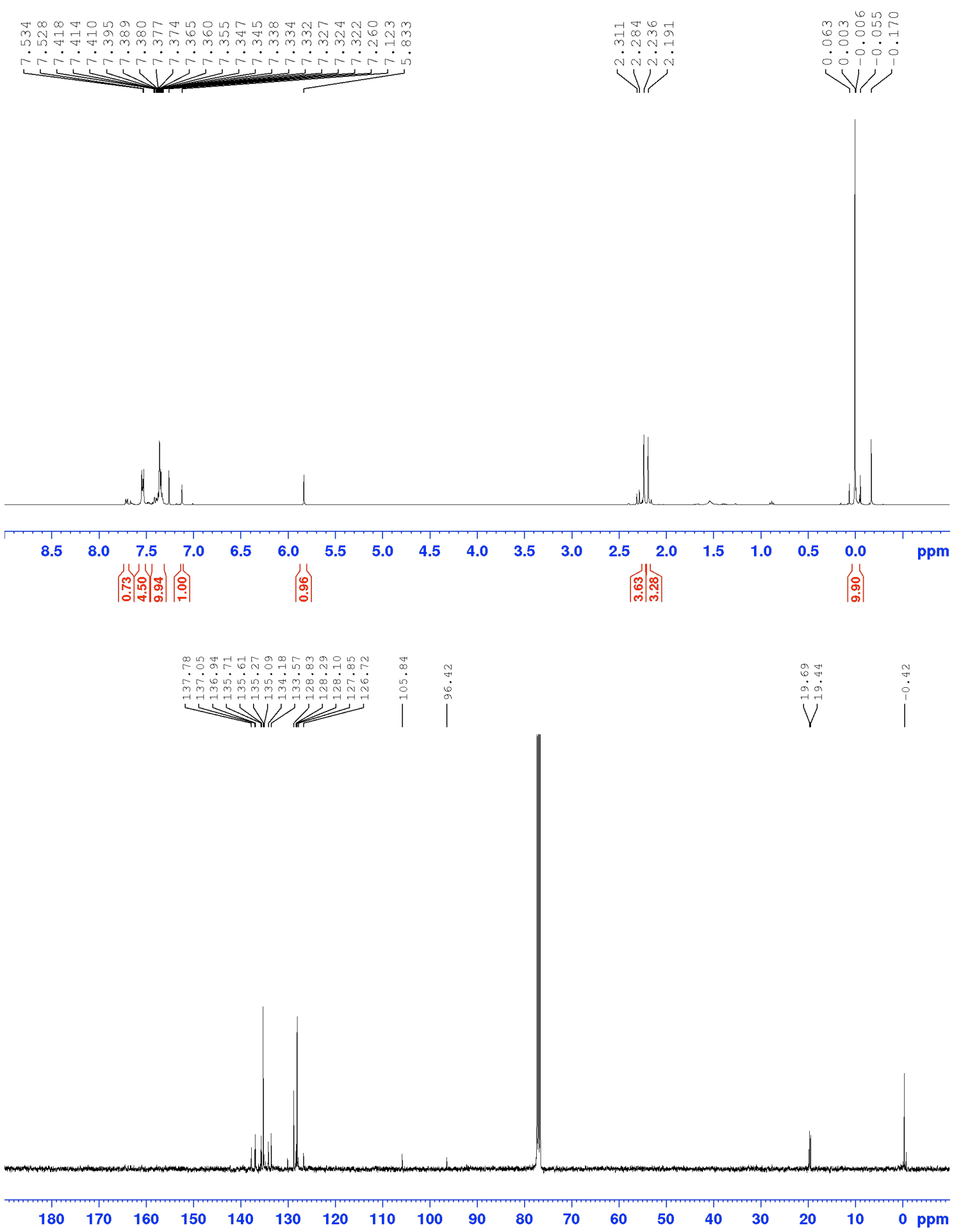


\section{2-[2-(Trimethylsilyl)ethynyl]-3-(diphenylgermyl)naphthalene (3d).}

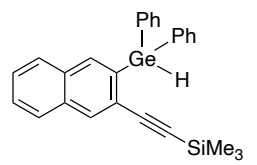

${ }^{1} \mathrm{H}$ NMR (300.13 MHz, $\left.\mathrm{CDCl}_{3}, \mathrm{CHCl}_{3}: \delta 7.26 \mathrm{ppm}\right),{ }^{13} \mathrm{C} \mathrm{NMR}\left(100.61 \mathrm{MHz}, \mathrm{CDCl}_{3}: \delta 77.0 \mathrm{ppm}\right)$

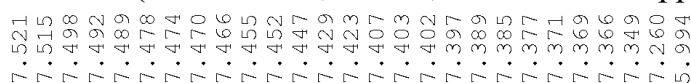
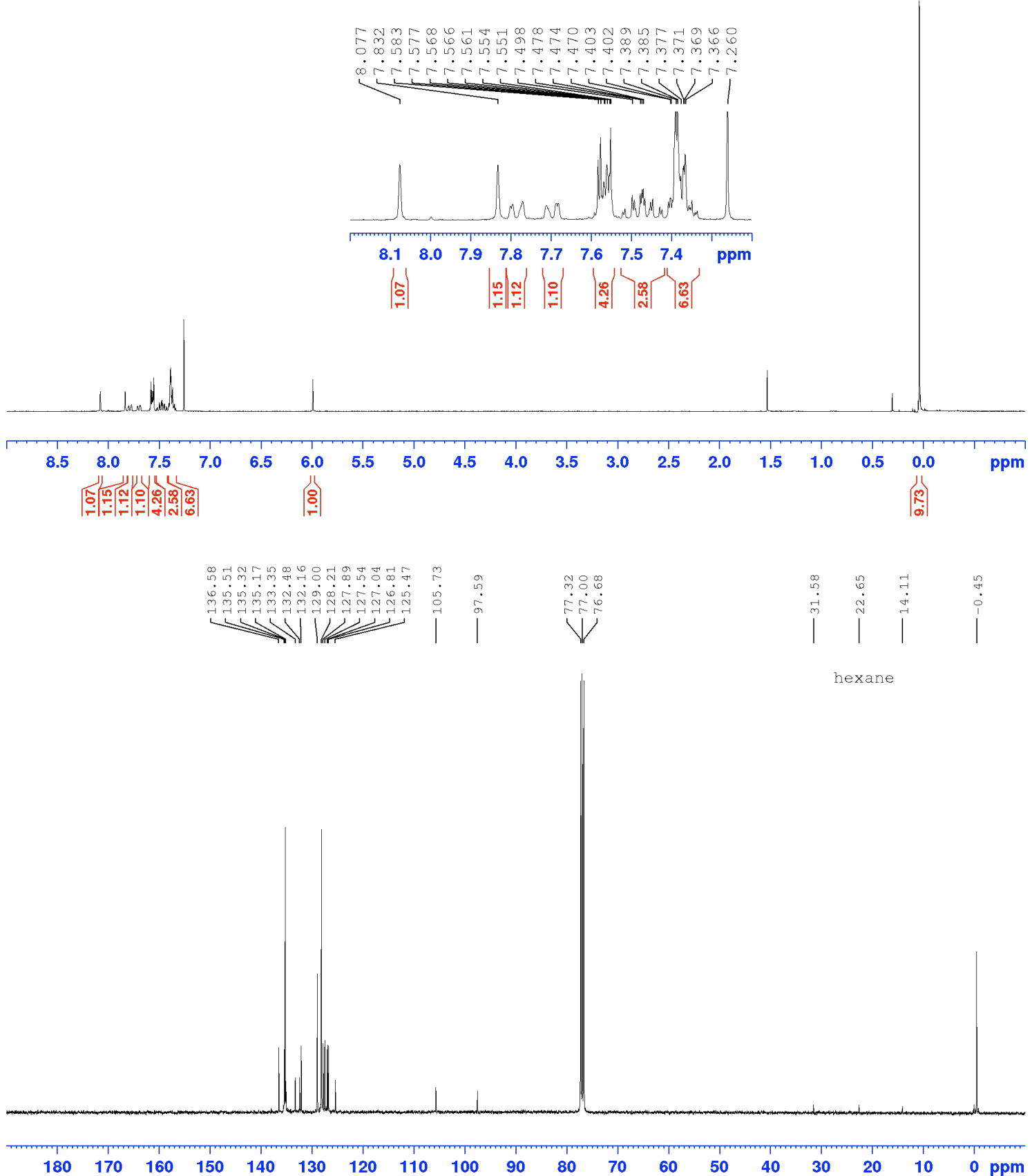
5-Fluoro-2-[2-(trimethylsilyl)ethynyl]-1-(diphenylgermyl)benzene (3e).<smiles>CSC#Cc1ccc(F)cc1C(c1ccccc1)c1ccccc1</smiles>

${ }^{1} \mathrm{H}$ NMR (300.13 MHz, $\left.\mathrm{CDCl}_{3}, \mathrm{CHCl}_{3}: \delta 7.26 \mathrm{ppm}\right),{ }^{13} \mathrm{C} \mathrm{NMR}\left(75.47 \mathrm{MHz}, \mathrm{CDCl}_{3}: \delta 77.0 \mathrm{ppm}\right)$
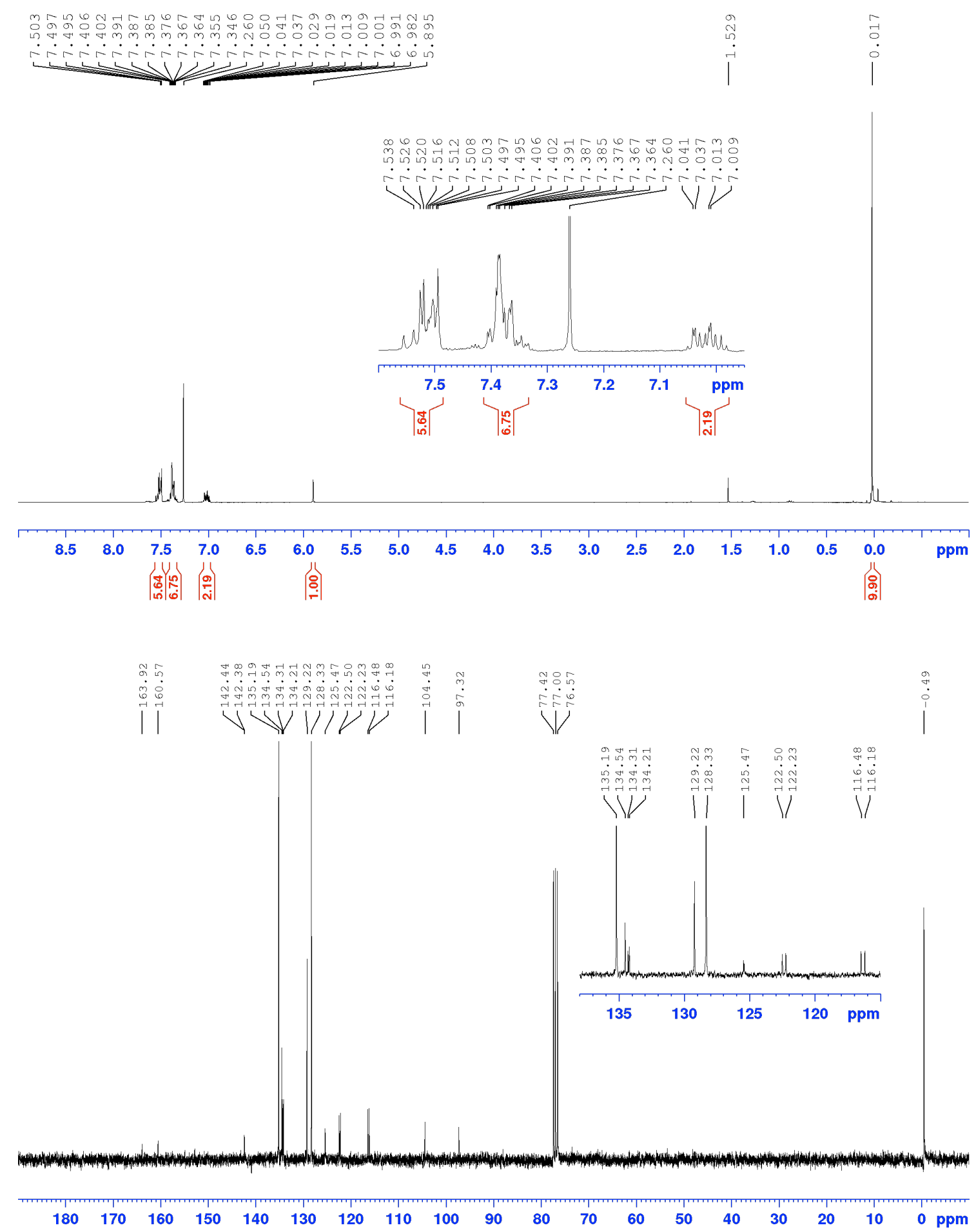
5-Chloro-2-[2-(trimethylsilyl)ethynyl]-1-(diphenylgermyl)benzene (3f).<smiles>[CH][Ge](c1ccccc1)(c1ccccc1)c1cc(Cl)ccc1C#CC</smiles>

${ }^{1} \mathrm{H}$ NMR (300.13 MHz, $\left.\mathrm{CDCl}_{3}, \mathrm{CHCl}_{3}: \delta 7.26 \mathrm{ppm}\right),{ }^{13} \mathrm{C} \mathrm{NMR}\left(100.61 \mathrm{MHz}, \mathrm{CDCl}_{3}: \delta 77.0 \mathrm{ppm}\right)$
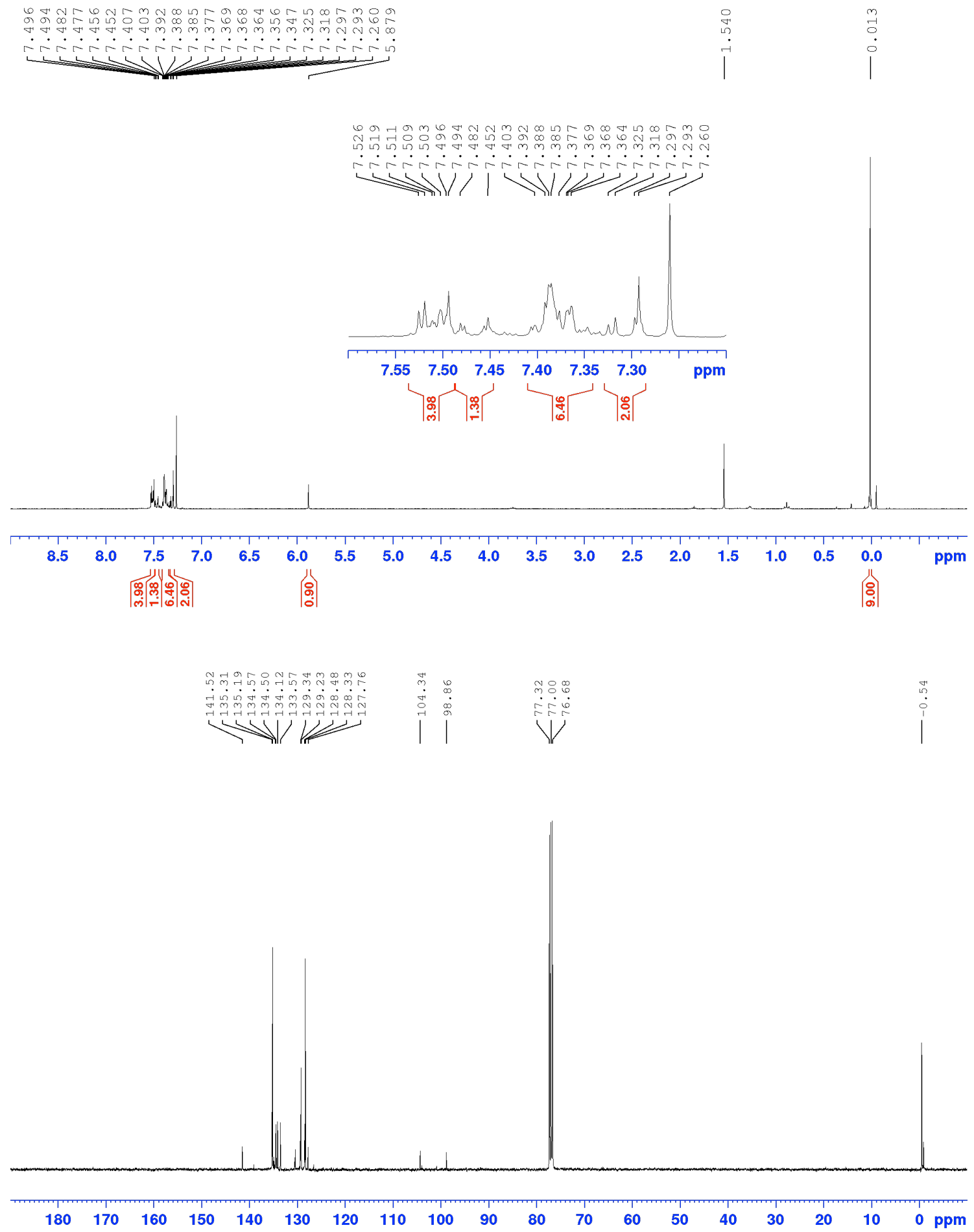
2-[2-(Diphenylmethylsilyl)ethynyl]-1-(diphenylgermyl)benzene (3g).

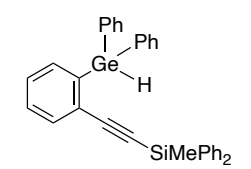

${ }^{1} \mathrm{H} \mathrm{NMR}\left(400.13 \mathrm{MHz}, \mathrm{CDCl}_{3}, \mathrm{CHCl}_{3}: \delta 7.26 \mathrm{ppm}\right),{ }^{13} \mathrm{C} \mathrm{NMR}\left(100.61 \mathrm{MHz}, \mathrm{CDCl}_{3}: \delta 77.0 \mathrm{ppm}\right)$
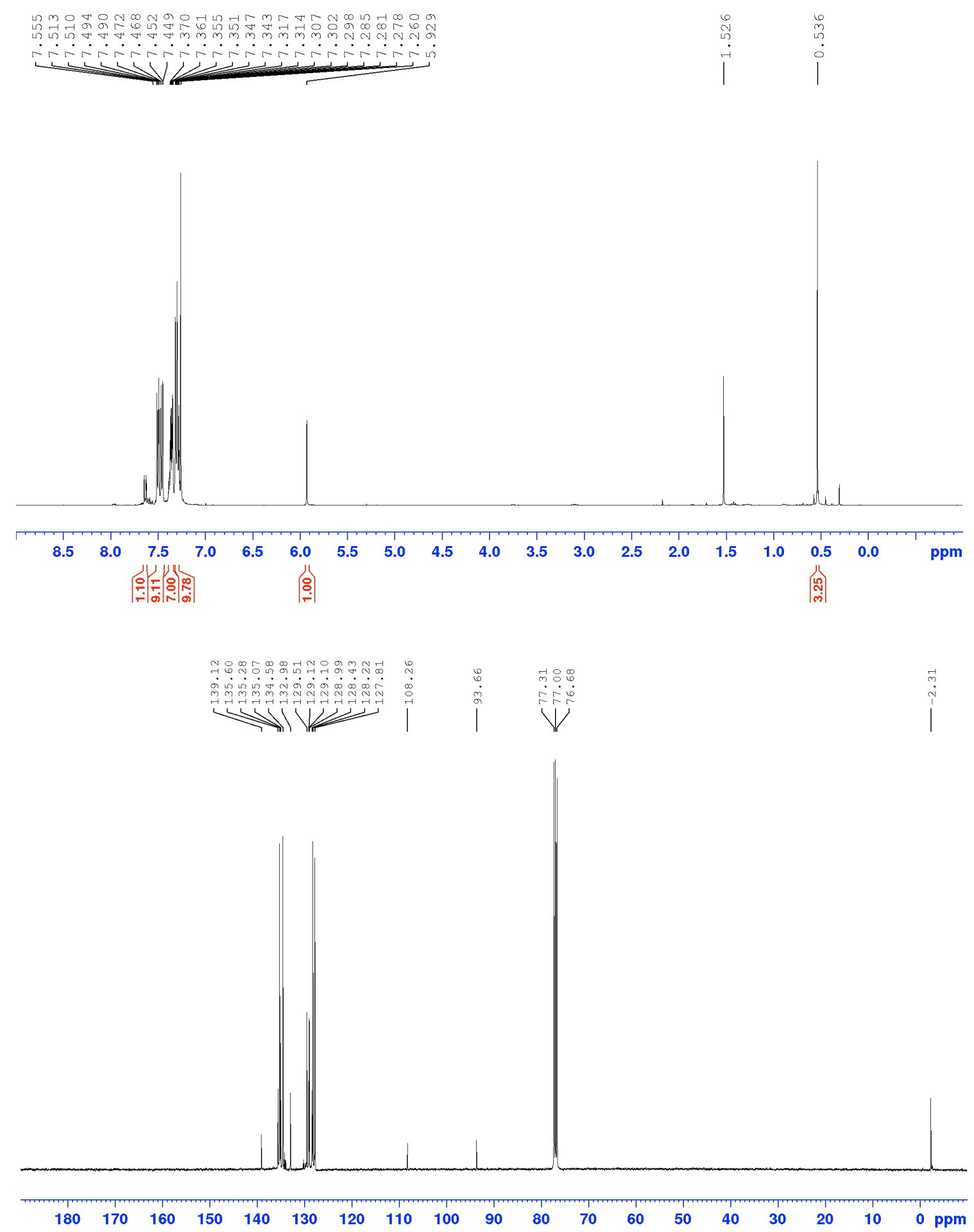
2-[2-(Triphenylsilyl)ethynyl]-1-(diphenylgermyl)benzene (3h).<smiles>C(#Cc1ccccc1OCc1ccccc1)c1ccccc1</smiles>

${ }^{1} \mathrm{H}$ NMR (400.13 MHz, $\left.\mathrm{CDCl}_{3}, \mathrm{CHCl}_{3}: \delta 7.26 \mathrm{ppm}\right),{ }^{13} \mathrm{C} \mathrm{NMR}\left(100.61 \mathrm{MHz}, \mathrm{CDCl}_{3}: \delta 77.0 \mathrm{ppm}\right)$
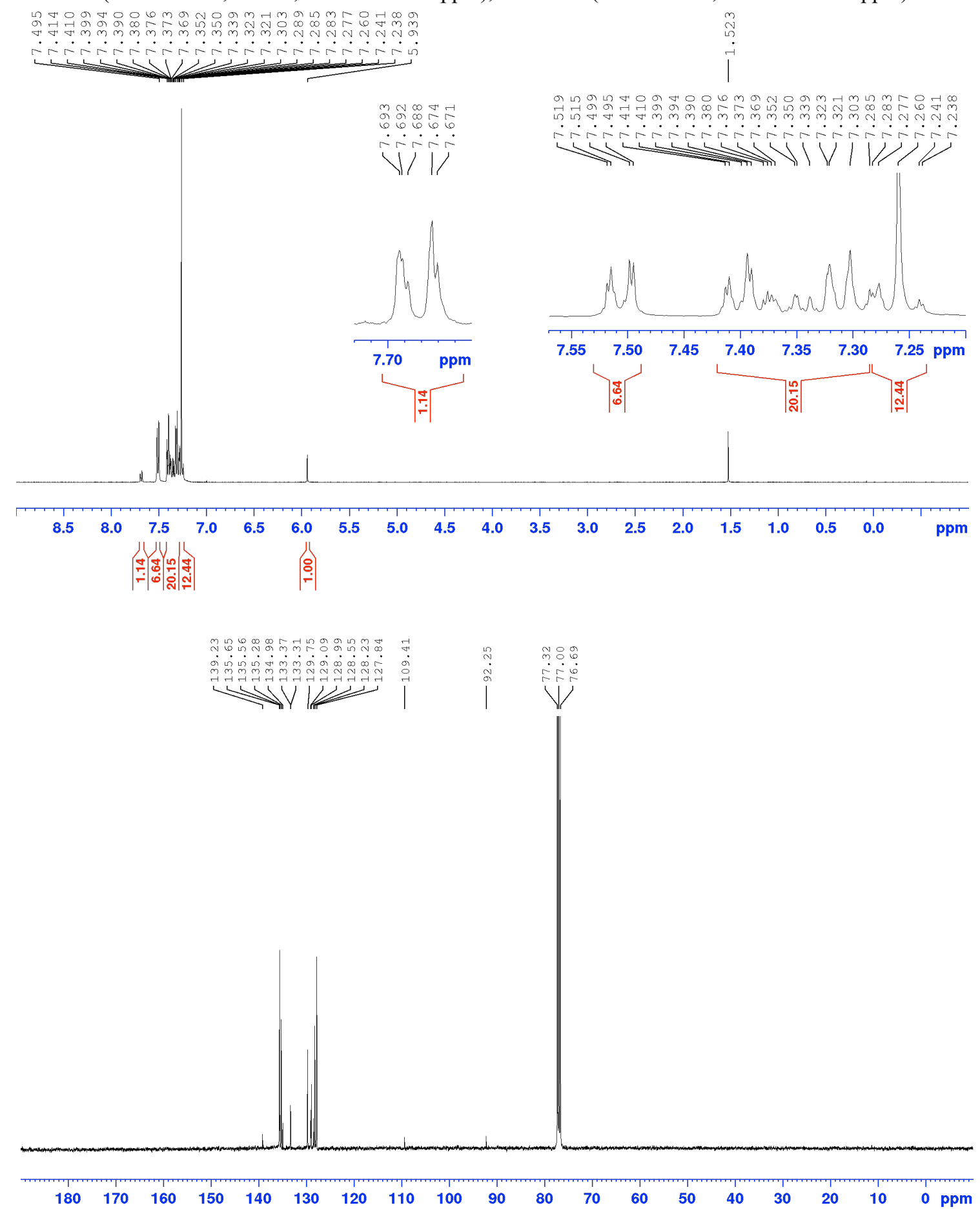
2,5-Bis(diphenylgermyl)-1,4-bis[2-(trimethylsilyl)ethynyl]benzene (3i).

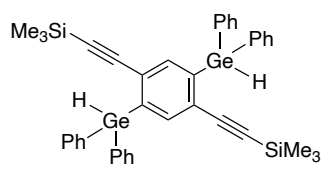

${ }^{1} \mathrm{H}$ NMR (300.13 MHz, $\left.\mathrm{CDCl}_{3}, \mathrm{CHCl}_{3}: \delta 7.26 \mathrm{ppm}\right),{ }^{13} \mathrm{C} \mathrm{NMR}\left(100.61 \mathrm{MHz}, \mathrm{CDCl}_{3}: \delta 77.0 \mathrm{ppm}\right)$
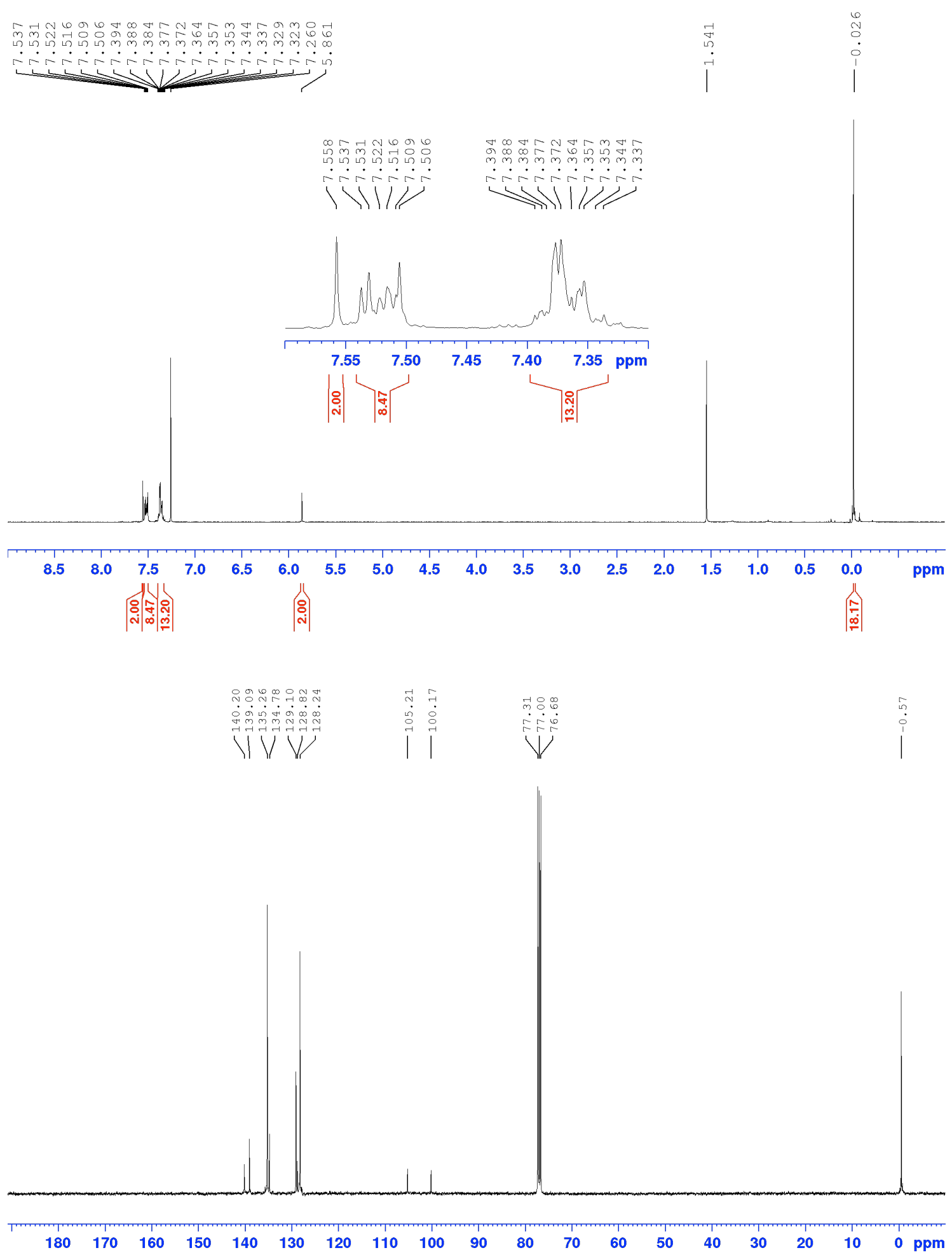
1,1-Diphenyl-2,3-dipropyl-5-(trimethylsilyl)-1H-germole (2a).

n-Pr

${ }^{1} \mathrm{H}$ NMR (300.13 MHz, $\left.\mathrm{CDCl}_{3}, \mathrm{CHCl}_{3}: \delta 7.26 \mathrm{ppm}\right),{ }^{13} \mathrm{C} \mathrm{NMR}\left(125.73 \mathrm{MHz}, \mathrm{CDCl}_{3}: \delta 77.0 \mathrm{ppm}\right)$
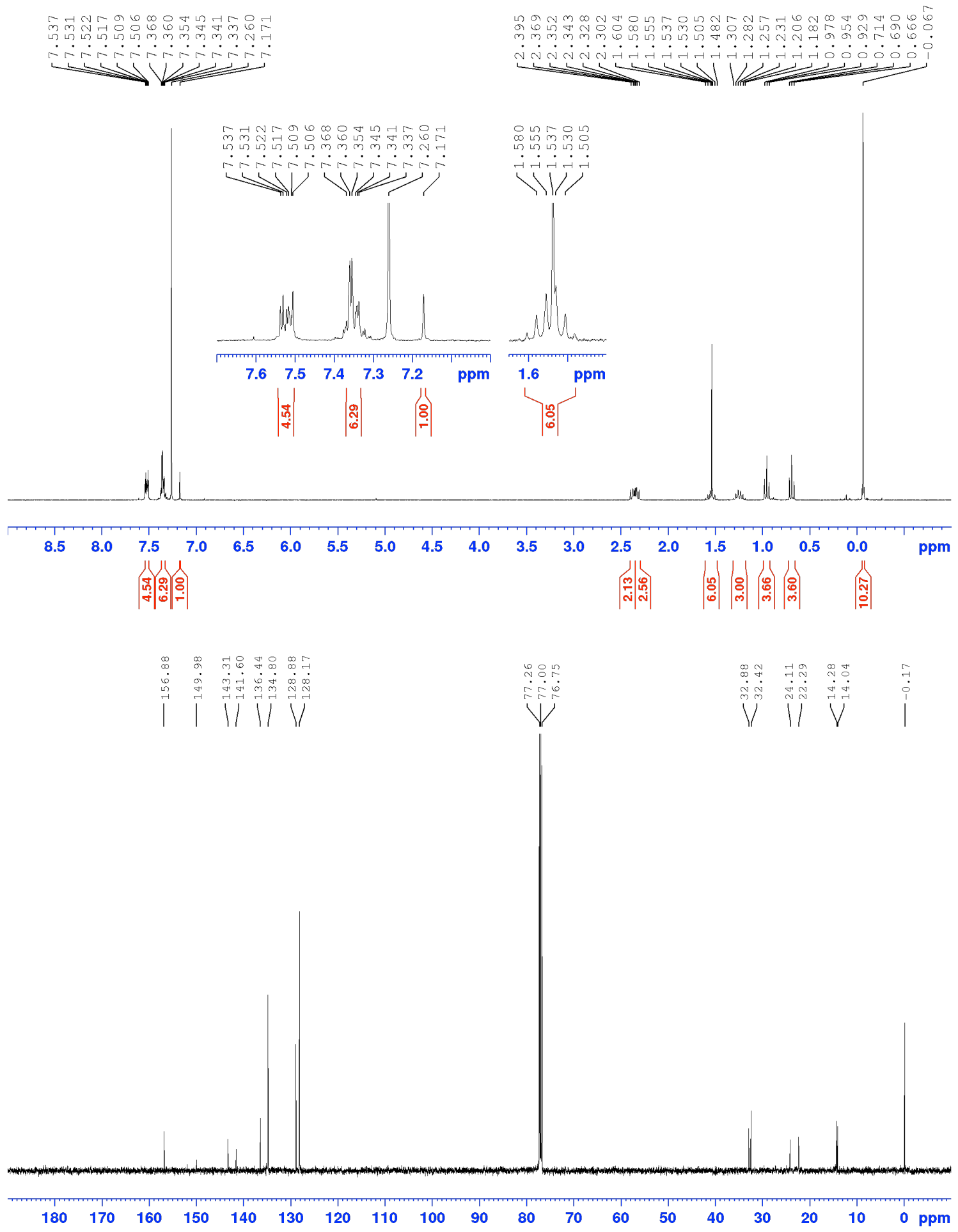


\section{1,1-Diphenyl-2-butyl-3-ethyl-5-(trimethylsilyl)-1 $H$-germole (2b).}

$$
\text { (- } \mathrm{SiMe}_{3}
$$

${ }^{1} \mathrm{H}$ NMR (300.13 MHz, $\left.\mathrm{CDCl}_{3}, \mathrm{CHCl}_{3}: \delta 7.26 \mathrm{ppm}\right),{ }^{13} \mathrm{C} \mathrm{NMR}\left(100.61 \mathrm{MHz}, \mathrm{CDCl}_{3}: \delta 77.0 \mathrm{ppm}\right)$
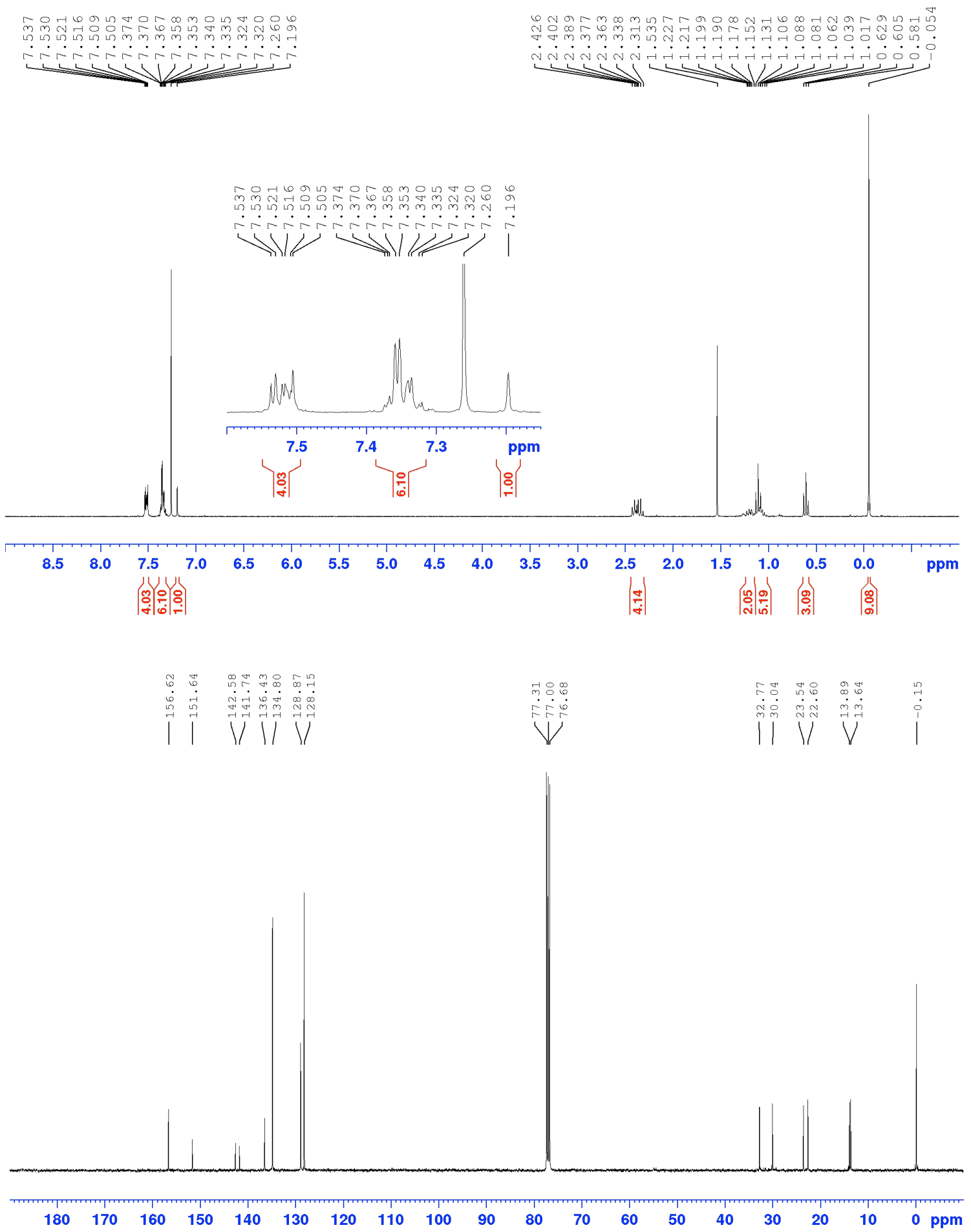
1,1-Diphenyl-2-(3-methylbutyl)-3-ethyl-5-(trimethylsilyl)-1 $H$-germole (2c).

Me

${ }^{1} \mathrm{H}$ NMR (300.13 MHz, $\left.\mathrm{CDCl}_{3}, \mathrm{CHCl}_{3}: \delta 7.26 \mathrm{ppm}\right),{ }^{13} \mathrm{C} \mathrm{NMR}\left(100.61 \mathrm{MHz}, \mathrm{CDCl}_{3}: \delta 77.0 \mathrm{ppm}\right)$

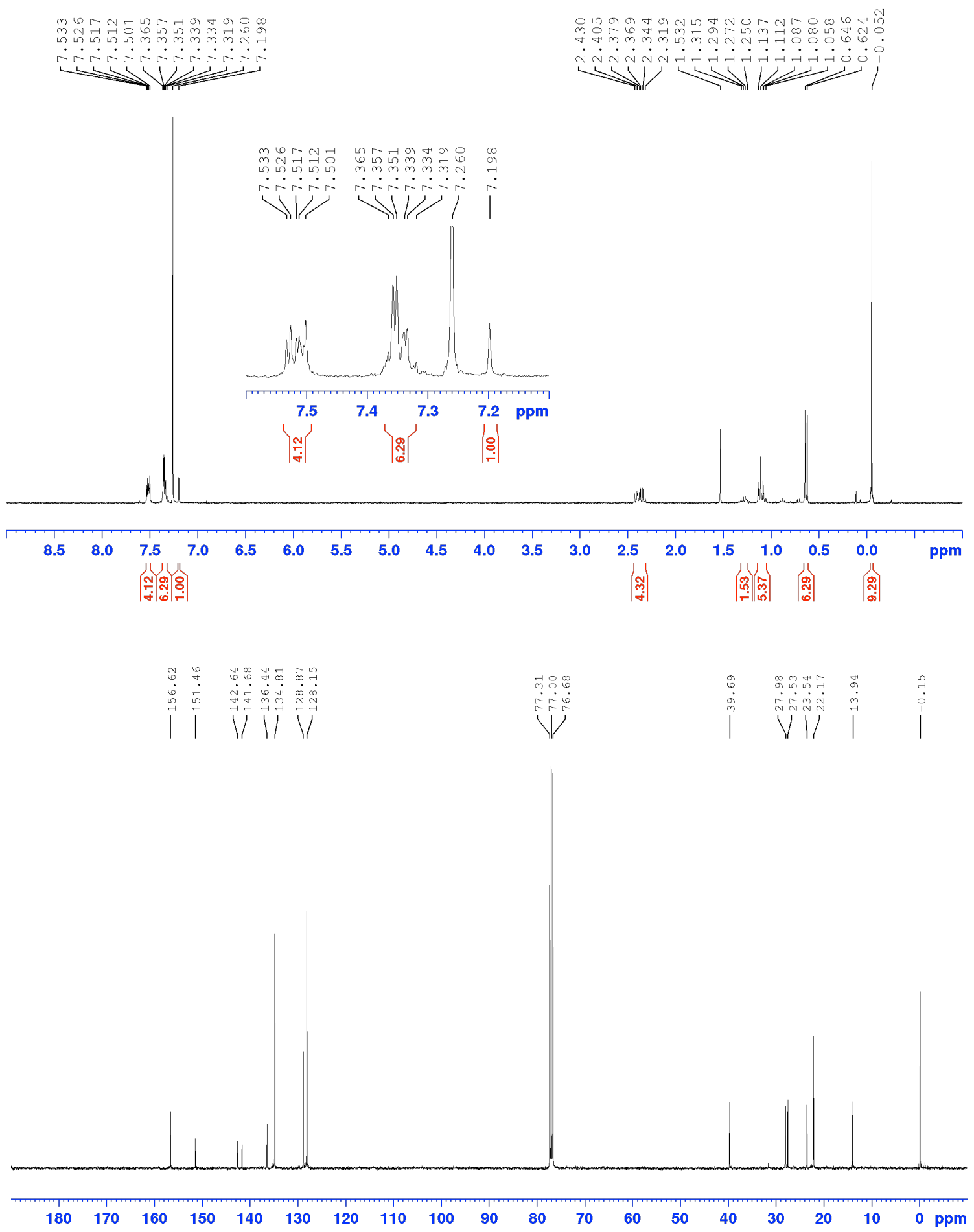




\section{1,1-Diphenyl-2-cyclohexyl-3-ethyl-5-(trimethylsilyl)-1H-germole (2d).}

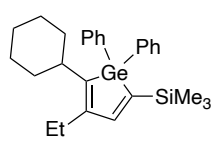

${ }^{1} \mathrm{H}$ NMR (300.13 MHz, $\left.\mathrm{CDCl}_{3}, \mathrm{CHCl}_{3}: \delta 7.26 \mathrm{ppm}\right),{ }^{13} \mathrm{C} \mathrm{NMR}\left(100.61 \mathrm{MHz}, \mathrm{CDCl}_{3}: \delta 77.0 \mathrm{ppm}\right)$

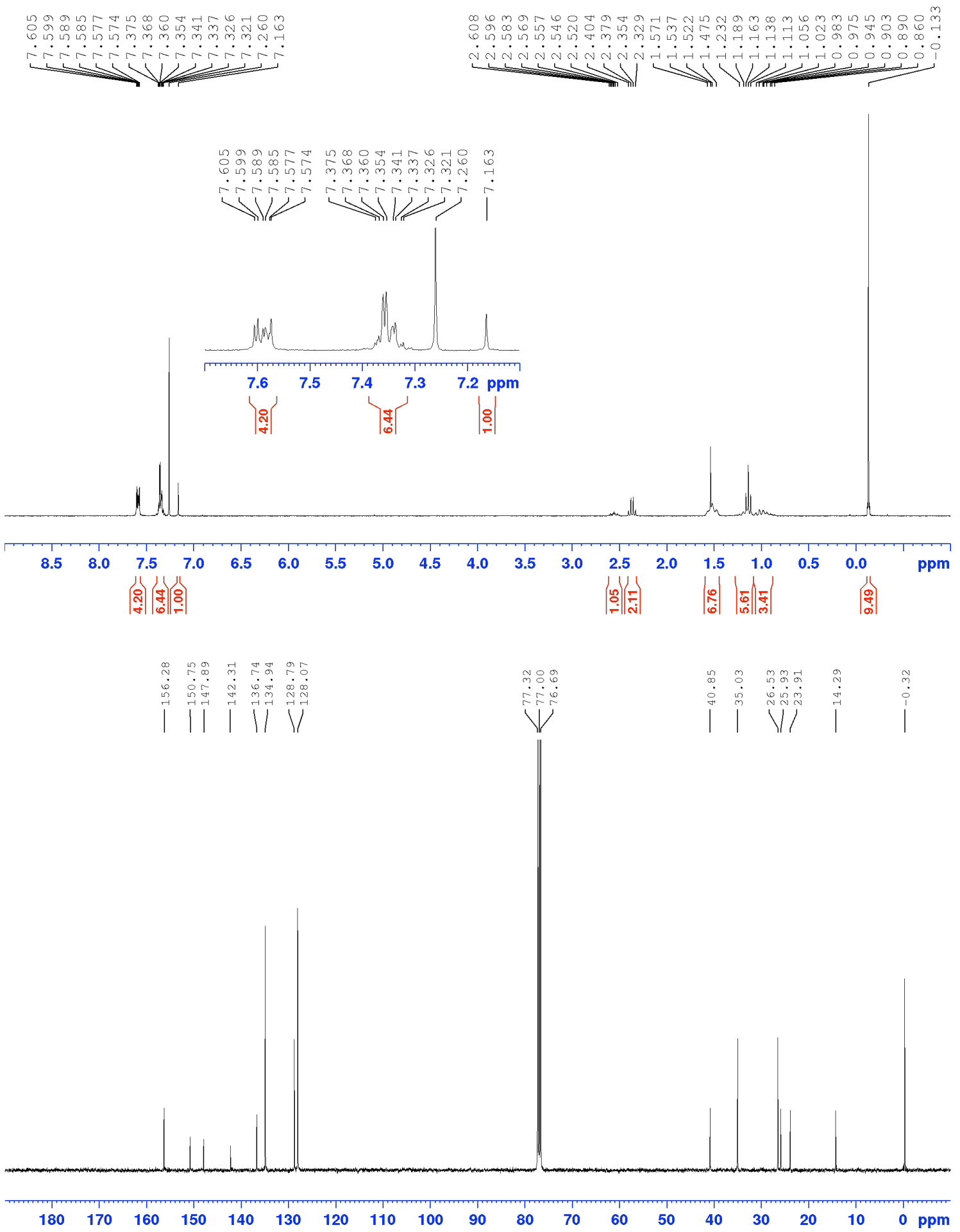


1,1-Diphenyl-2-trimethylsilyl-4,5,6,7-tetrahydro-1 $H$-benzo[b]germole (2e).<smiles></smiles>

${ }^{1} \mathrm{H}$ NMR $\left(400.13 \mathrm{MHz}, \mathrm{CDCl}_{3}, \mathrm{CHCl}_{3}: \delta 7.26 \mathrm{ppm}\right),{ }^{13} \mathrm{C} \mathrm{NMR}\left(100.61 \mathrm{MHz}, \mathrm{CDCl}_{3}: \delta 77.0 \mathrm{ppm}\right)$
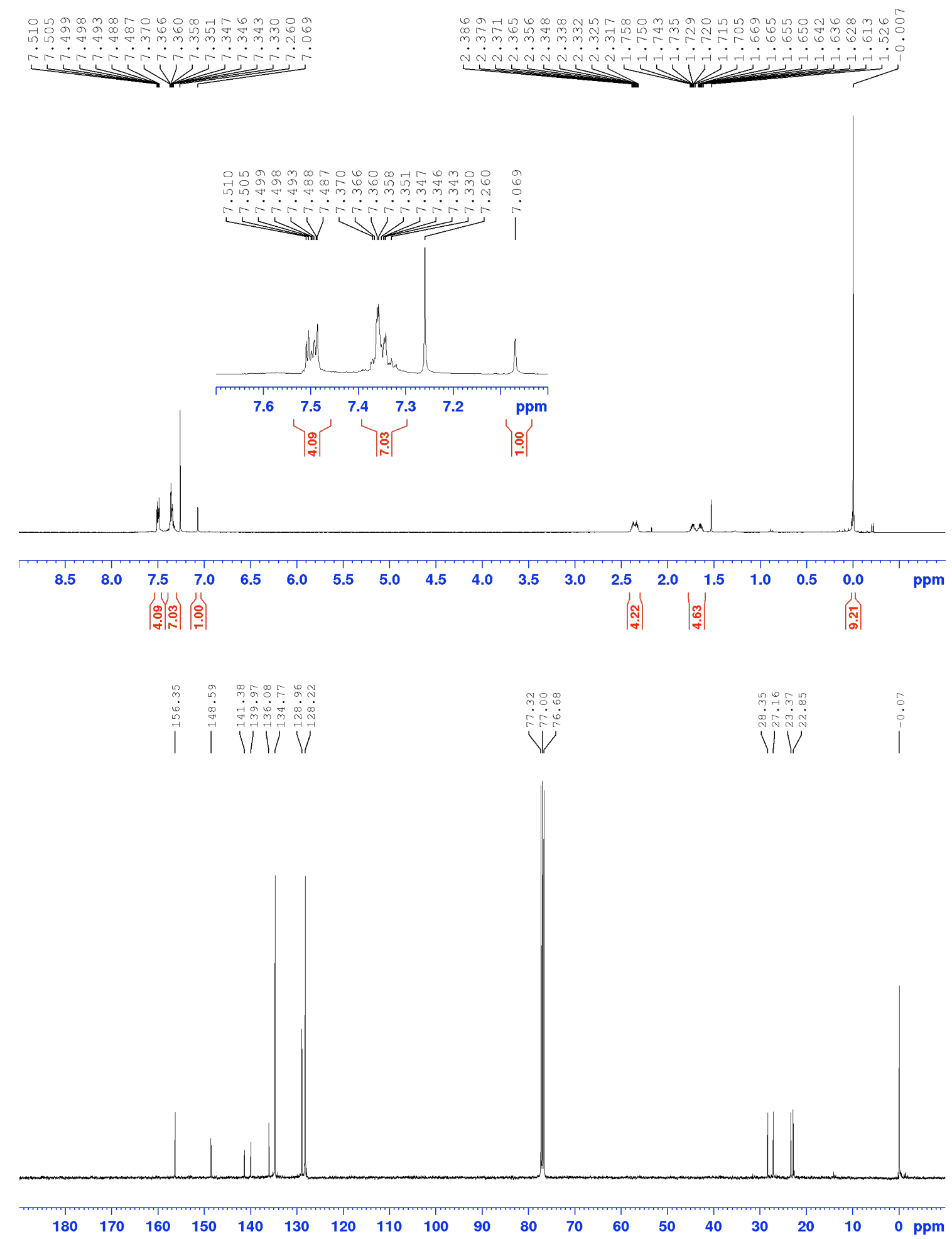
1,1-Diphenyl-2-phenyl-3-ethyl-5-(trimethylsilyl)-1 $H$-germole (2f).

Ph

${ }^{1} \mathrm{H}$ NMR (400.13 MHz, $\left.\mathrm{CDCl}_{3}, \mathrm{CHCl}_{3}: \delta 7.26 \mathrm{ppm}\right),{ }^{13} \mathrm{C} \mathrm{NMR}\left(100.61 \mathrm{MHz}, \mathrm{CDCl}_{3}: \delta 77.0 \mathrm{ppm}\right)$
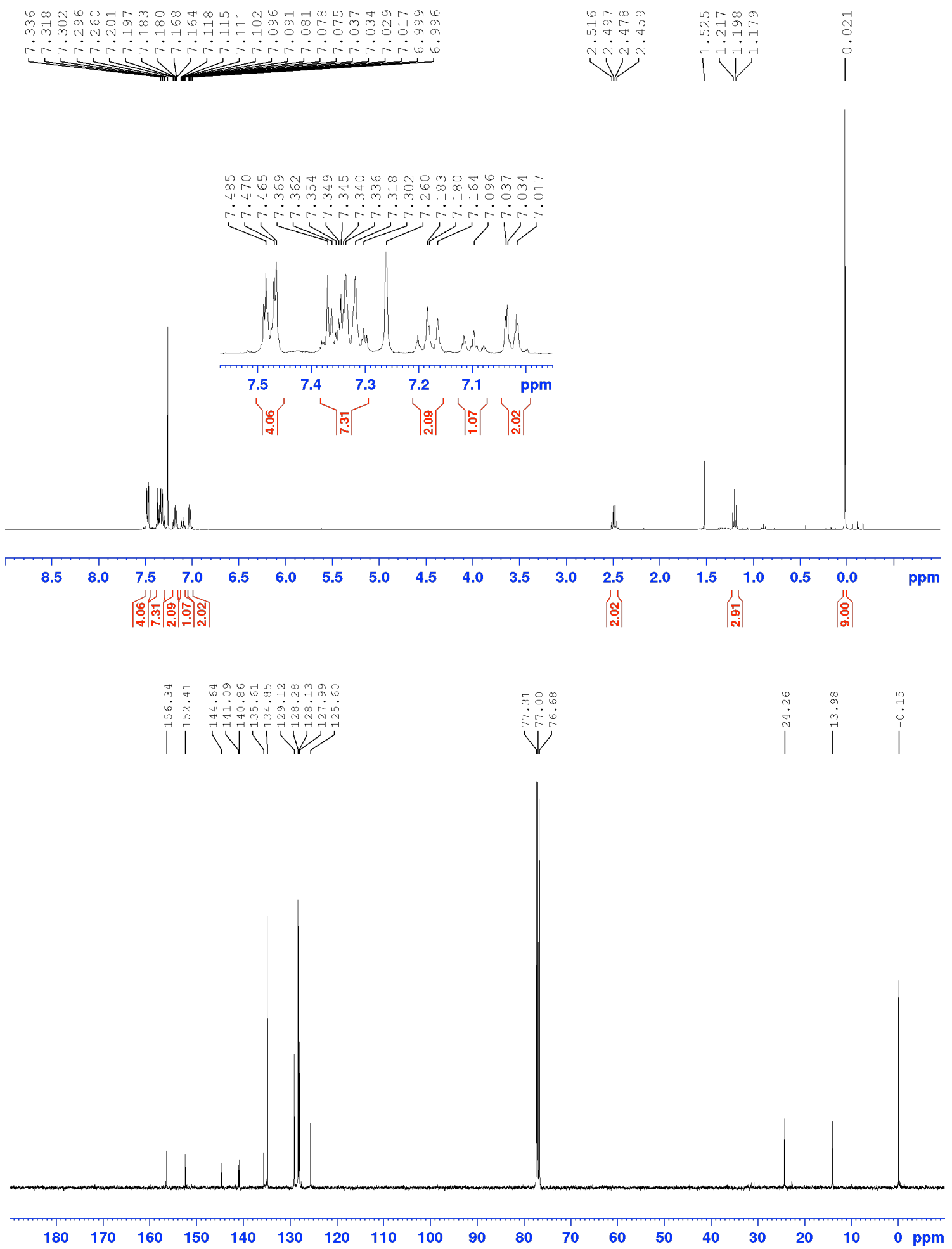
1,1-Diphenyl-2-trimethylsilyl-1 $H$-benzo[b]germole (4a).

${ }^{\mathrm{Ph}} \mathrm{Sime}_{3}$

${ }^{1} \mathrm{H}$ NMR (300.13 MHz, $\left.\mathrm{CDCl}_{3}, \mathrm{CHCl}_{3}: \delta 7.26 \mathrm{ppm}\right),{ }^{13} \mathrm{C} \mathrm{NMR}\left(75.47 \mathrm{MHz}, \mathrm{CDCl}_{3}: \delta 77.0 \mathrm{ppm}\right)$
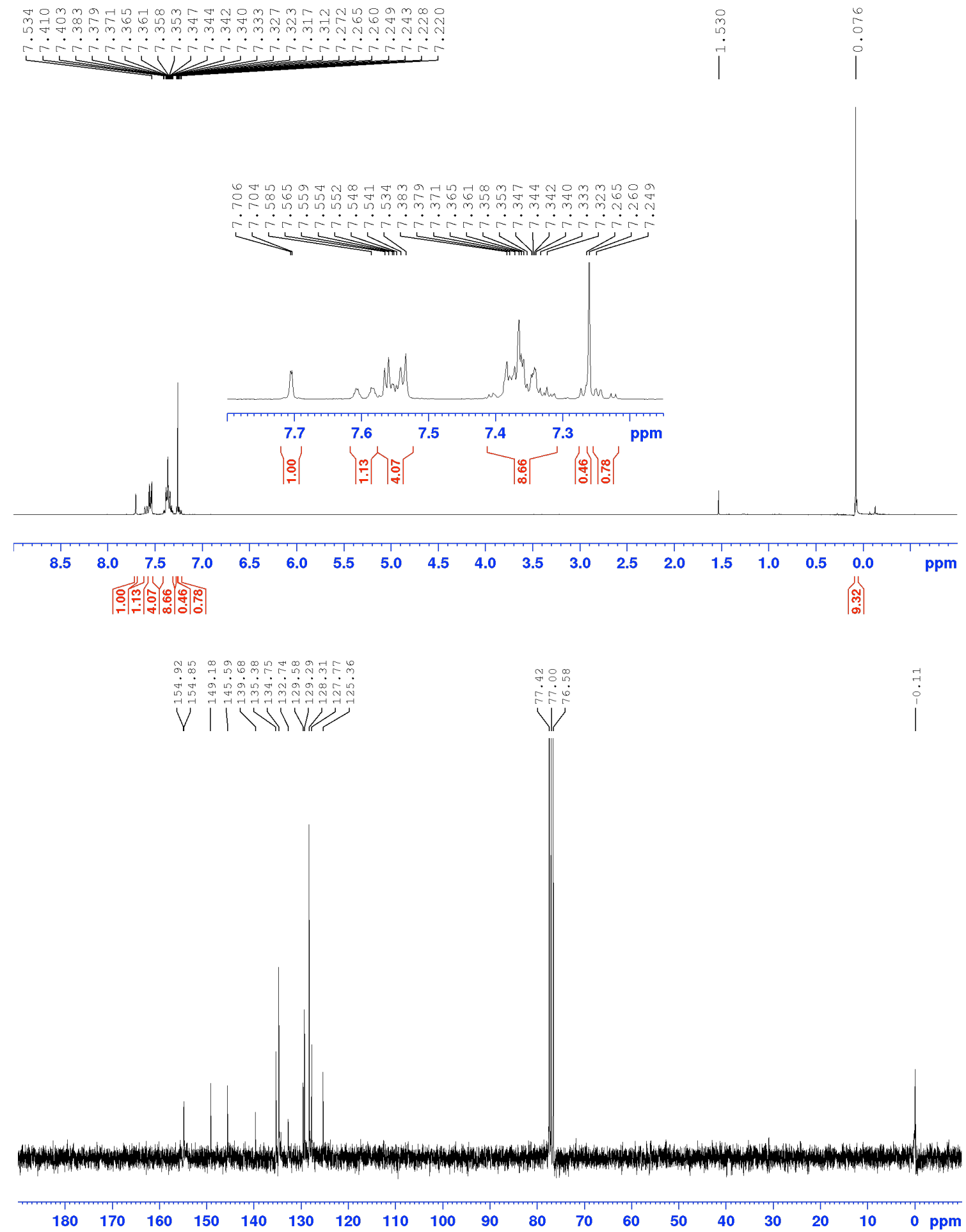
(E)-1-(Diphenylgermyl)-2-(2-trimethylsilylethenyl)benzene (5a). A mixture with 3a $(\mathbf{5 a}=71 \%, 3 \mathbf{a}=$ $28 \%)$.

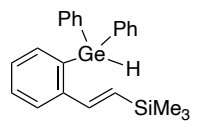

${ }^{1} \mathrm{H} \mathrm{NMR}\left(400.13 \mathrm{MHz}, \mathrm{CDCl}_{3}, \mathrm{CHCl}_{3}: \delta 7.26 \mathrm{ppm}\right),{ }^{13} \mathrm{C} \mathrm{NMR}\left(100.61 \mathrm{MHz}, \mathrm{CDCl}_{3}: \delta 77.0 \mathrm{ppm}\right)$
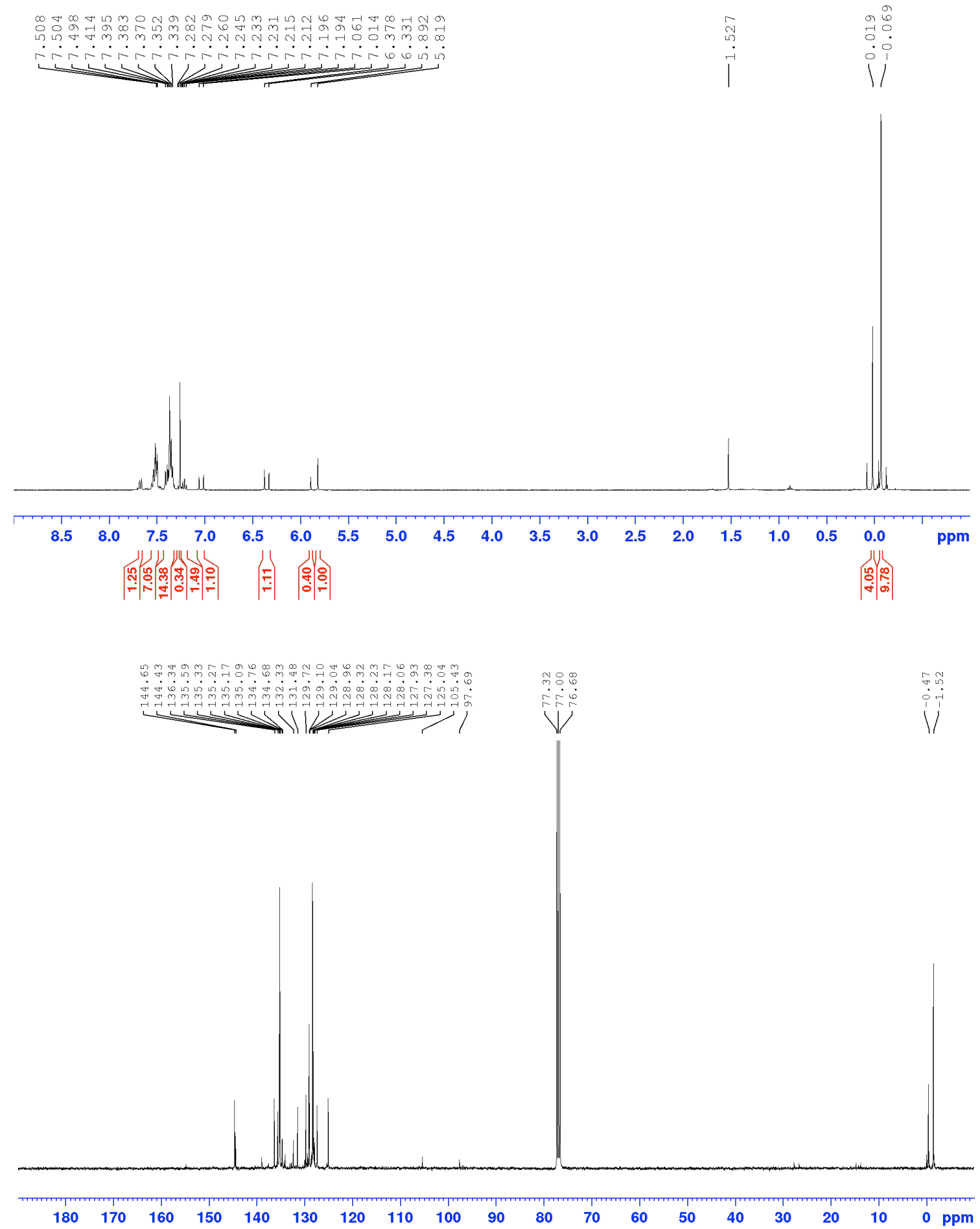
6-Methyl-1,1-diphenyl-2-trimethylsilyl-1 $H$-benzo[b]germole (4b).

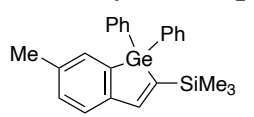

${ }^{1} \mathrm{H} \mathrm{NMR}\left(300.13 \mathrm{MHz}, \mathrm{CDCl}_{3}, \mathrm{CHCl}_{3}: \delta 7.26 \mathrm{ppm}\right),{ }^{13} \mathrm{C} \mathrm{NMR}\left(75.47 \mathrm{MHz}, \mathrm{CDCl}_{3}: \delta 77.0 \mathrm{ppm}\right)$
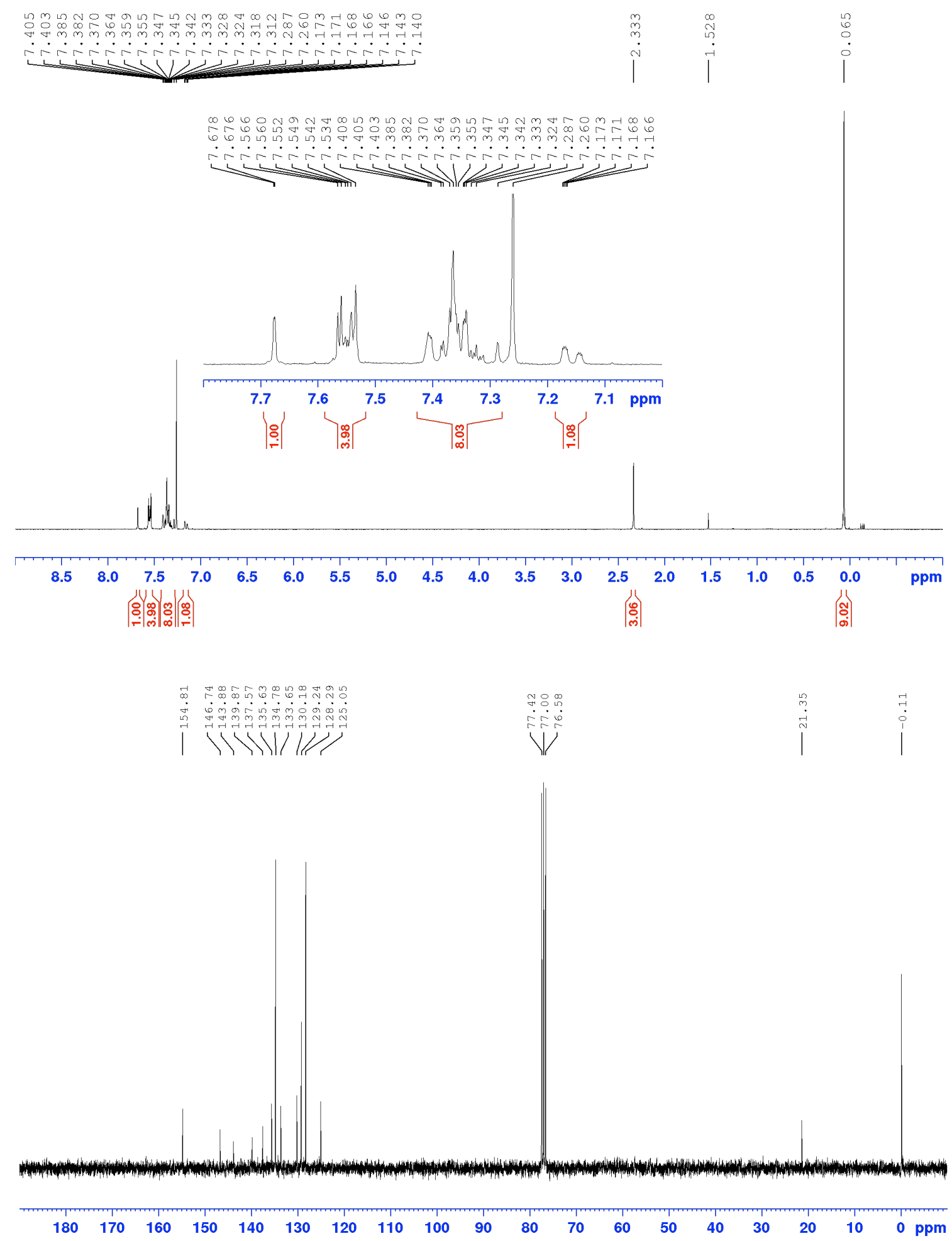
5,6-Dimethyl-1,1-diphenyl-2-trimethylsilyl-1H-benzo[b]germole (4c).

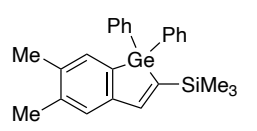

${ }^{1} \mathrm{H}$ NMR (400.13 MHz, $\left.\mathrm{CDCl}_{3}, \mathrm{CHCl}_{3}: \delta 7.26 \mathrm{ppm}\right),{ }^{13} \mathrm{C} \mathrm{NMR}\left(100.61 \mathrm{MHz}, \mathrm{CDCl}_{3}: \delta 77.0 \mathrm{ppm}\right)$
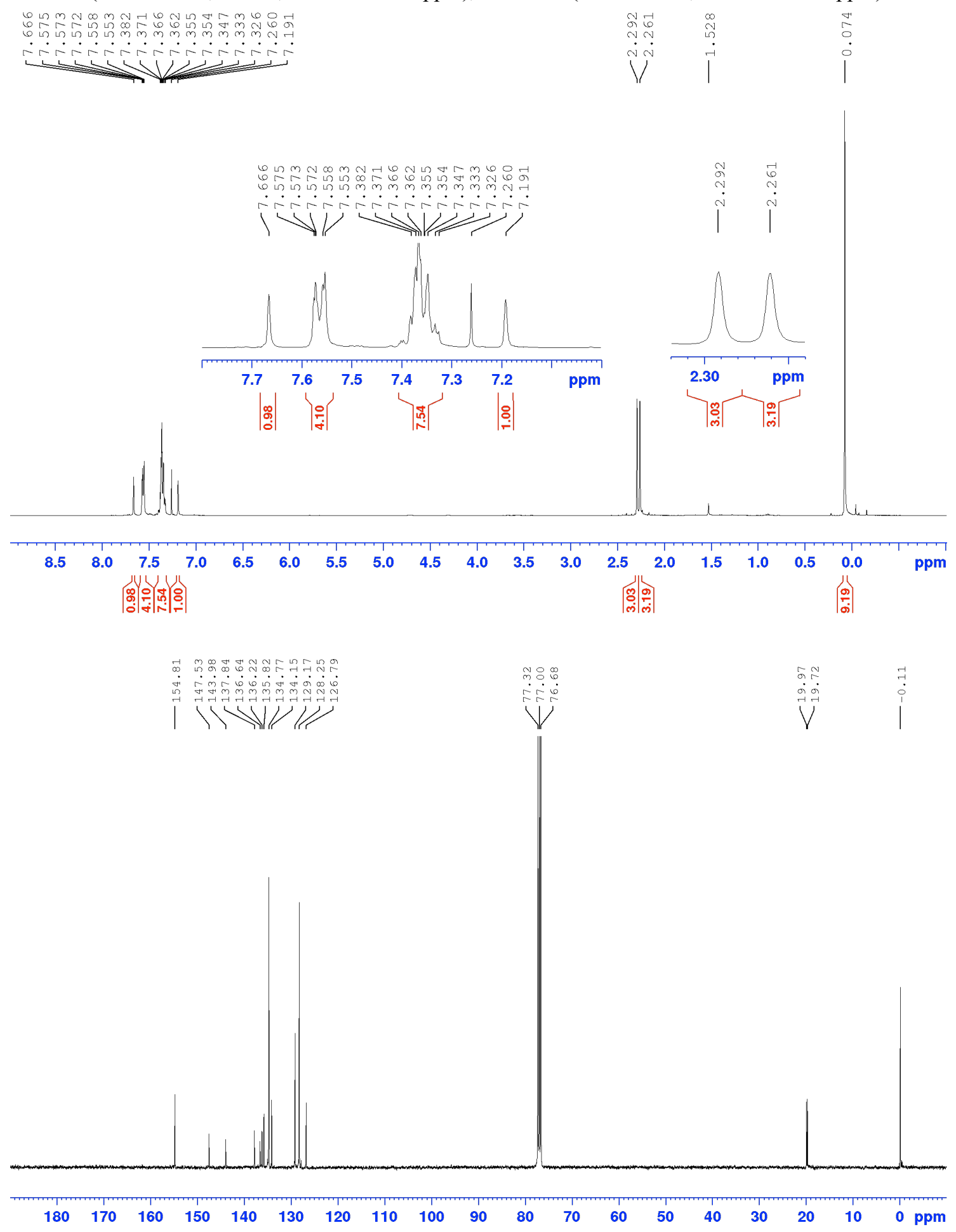
2-trimethylsilyl-1,1-diphenyl-1H-naphtho[2,3-b]germole (4d).

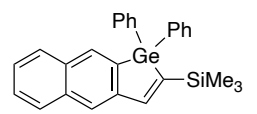

${ }^{1} \mathrm{H}$ NMR (400.13 MHz, $\left.\mathrm{CDCl}_{3}, \mathrm{CHCl}_{3}: \delta 7.26 \mathrm{ppm}\right),{ }^{13} \mathrm{C} \mathrm{NMR}\left(100.61 \mathrm{MHz}, \mathrm{CDCl}_{3}: \delta 77.0 \mathrm{ppm}\right)$
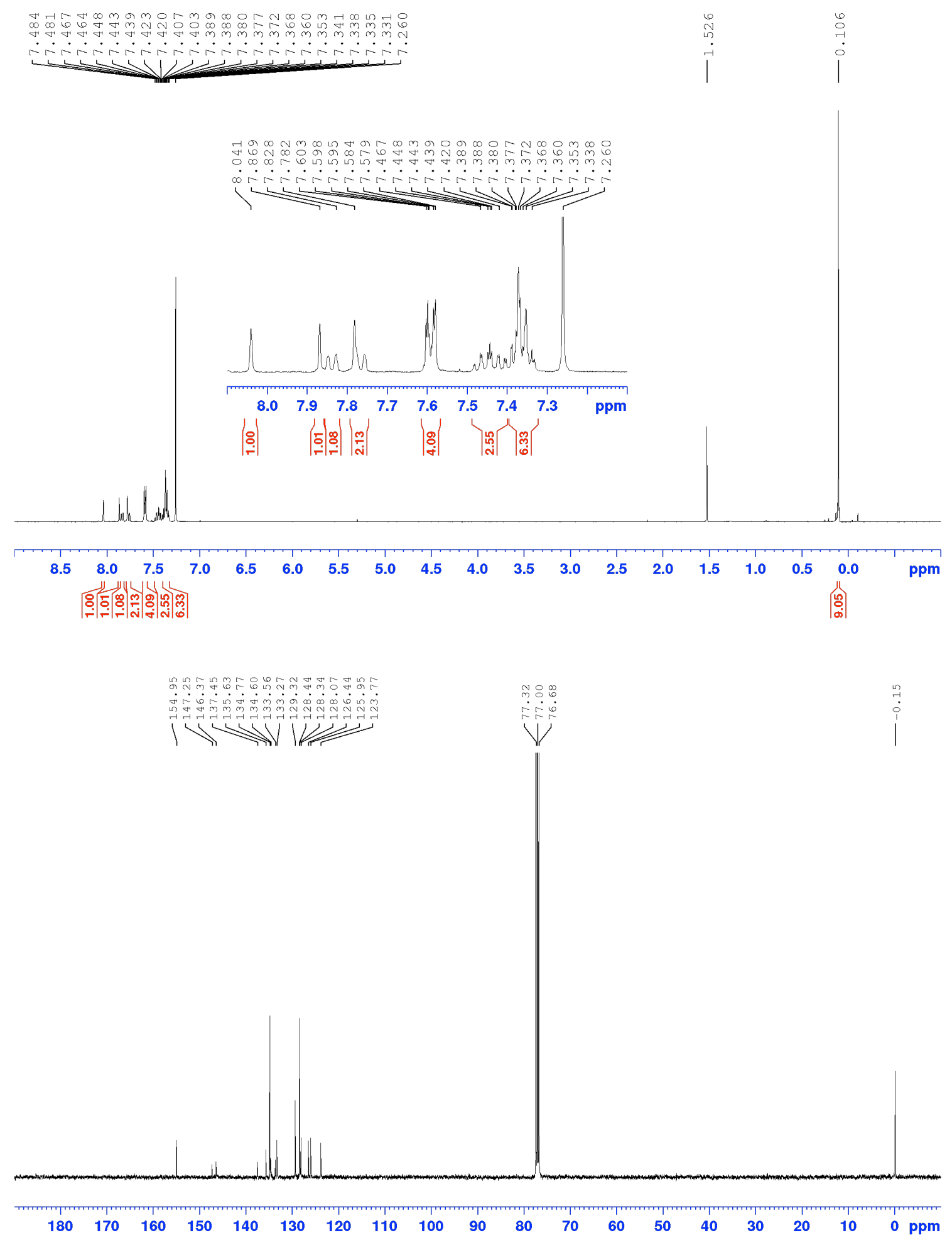
6-Fluoro-1,1-diphenyl-2-trimethylsilyl-1H-benzo[b]germole (4e).<smiles></smiles>

${ }^{1} \mathrm{H}$ NMR (300.13 MHz, $\left.\mathrm{CDCl}_{3}, \mathrm{CHCl}_{3}: \delta 7.26 \mathrm{ppm}\right),{ }^{13} \mathrm{C} \mathrm{NMR}\left(75.47 \mathrm{MHz}, \mathrm{CDCl}_{3}: \delta 77.0 \mathrm{ppm}\right)$
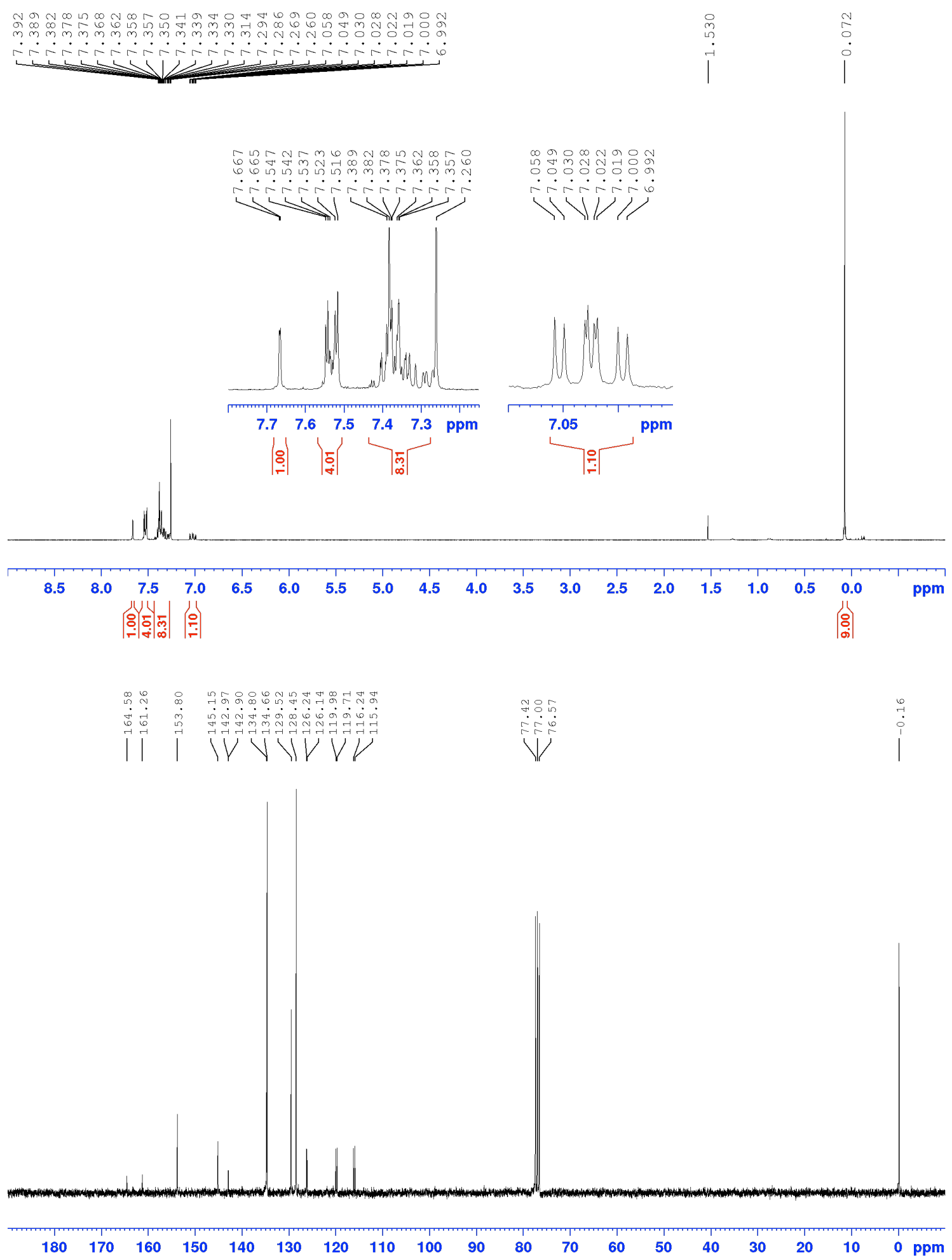
6-Chloro-1,1-diphenyl-2-trimethylsilyl-1H-benzo[b]germole (4f).<smiles>C[N+](C)(C)C1=Cc2ccc(Cl)cc2[Ge]1(c1ccccc1)c1ccccc1</smiles>

${ }^{1} \mathrm{H}$ NMR (300.13 MHz, $\left.\mathrm{CDCl}_{3}, \mathrm{CHCl}_{3}: \delta 7.26 \mathrm{ppm}\right),{ }^{13} \mathrm{C} \mathrm{NMR}\left(100.61 \mathrm{MHz}, \mathrm{CDCl}_{3}: \delta 77.0 \mathrm{ppm}\right)$
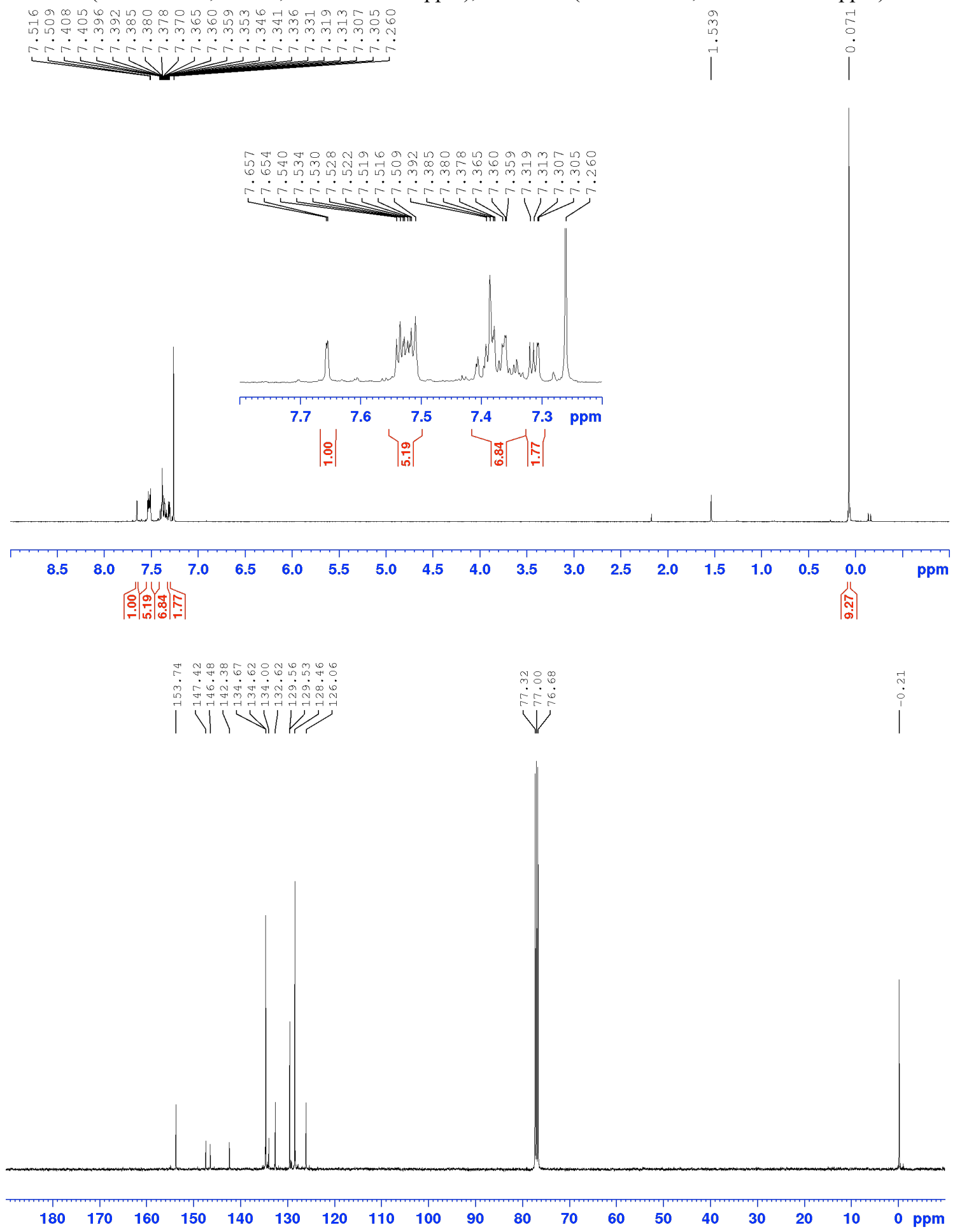
1,1-Diphenyl-2-methyl(diphenyl)silyl-1H-benzo[b]germole (4g).

$$
\text { (- }{ }^{2}
$$

${ }^{1} \mathrm{H}$ NMR (400.13 MHz, $\left.\mathrm{CDCl}_{3}, \mathrm{CHCl}_{3}: \delta 7.26 \mathrm{ppm}\right),{ }^{13} \mathrm{C} \mathrm{NMR}\left(100.61 \mathrm{MHz}, \mathrm{CDCl}_{3}: \delta 77.0 \mathrm{ppm}\right)$
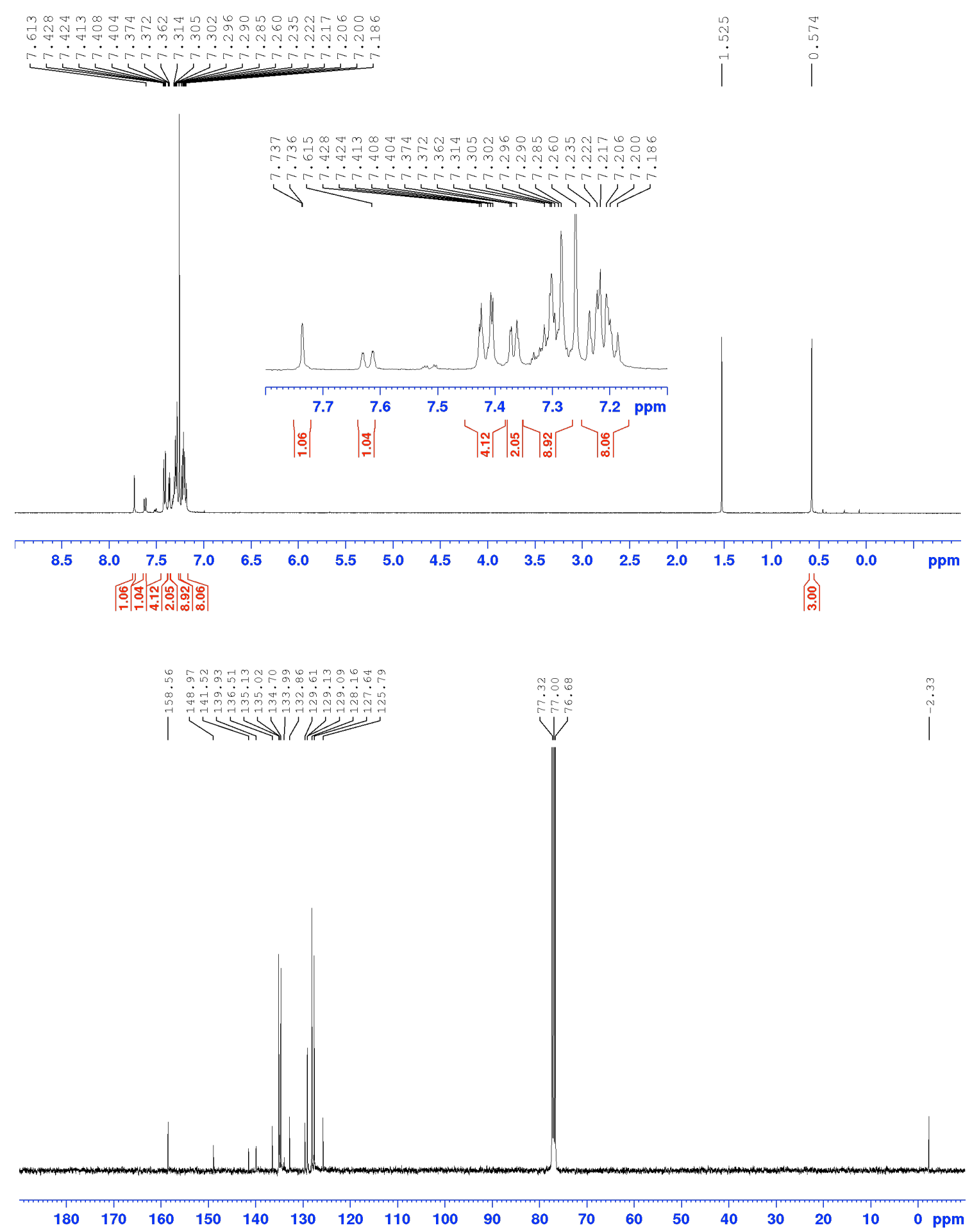
1,1-Diphenyl-2-triphenylsilyl-1 $H$-benzo[b]germole (4h).

(1)éph

${ }^{1} \mathrm{H}$ NMR (300.13 MHz, $\left.\mathrm{CDCl}_{3}, \mathrm{CHCl}_{3}: \delta 7.26 \mathrm{ppm}\right),{ }^{13} \mathrm{C} \mathrm{NMR}\left(100.61 \mathrm{MHz}, \mathrm{CDCl}_{3}: \delta 77.0 \mathrm{ppm}\right)$
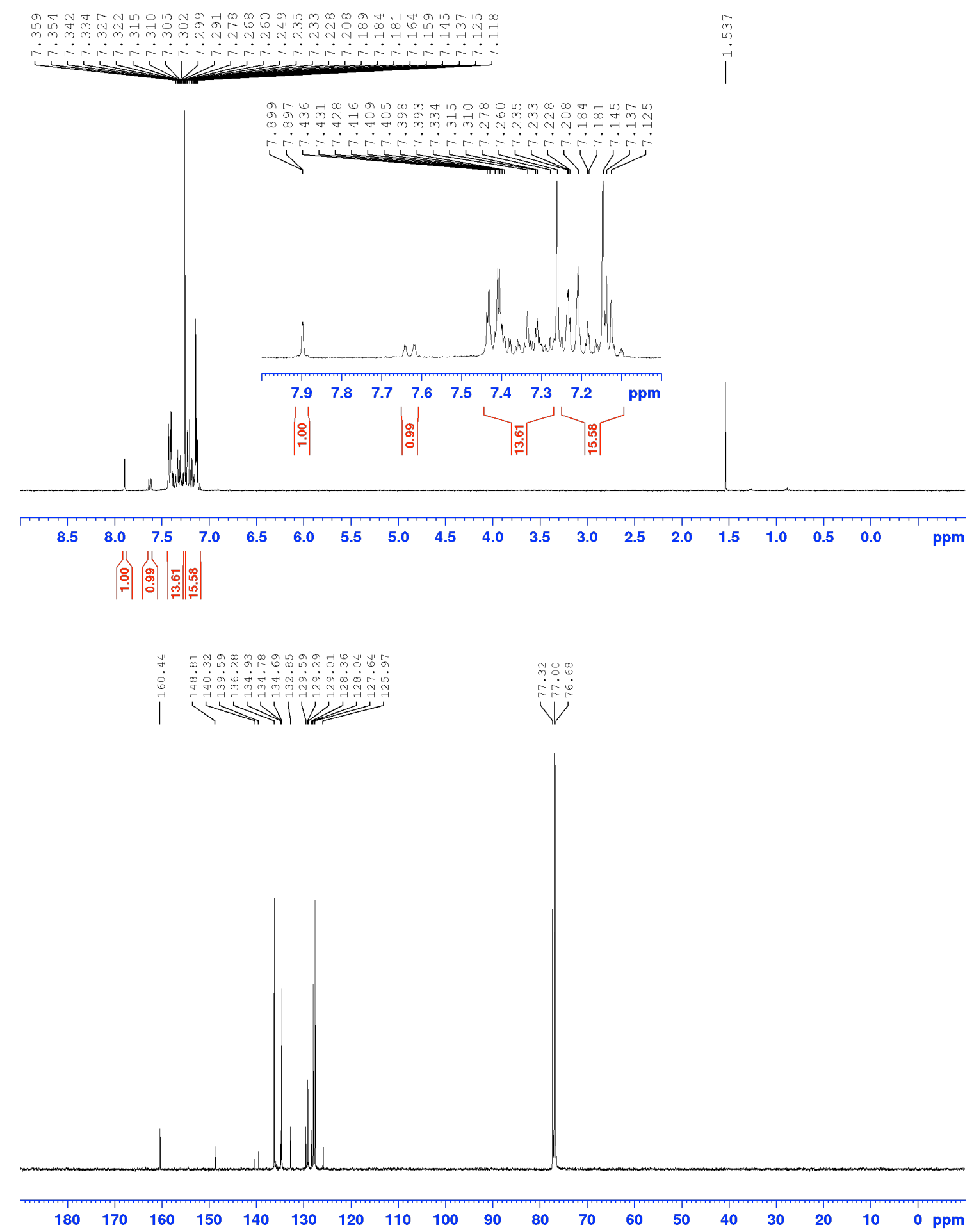
1,5-Dihydro-1,1,5,5-tetraphenyl-2,6-bis(trimethylsilyl)-1,5-digerma-s-indacene (4i).

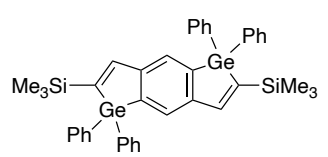

${ }^{1} \mathrm{H}$ NMR (300.13 MHz, $\left.\mathrm{CDCl}_{3}, \mathrm{CHCl}_{3}: \delta 7.26 \mathrm{ppm}\right),{ }^{13} \mathrm{C} \mathrm{NMR}\left(100.61 \mathrm{MHz}, \mathrm{CDCl}_{3}: \delta 77.0 \mathrm{ppm}\right)$
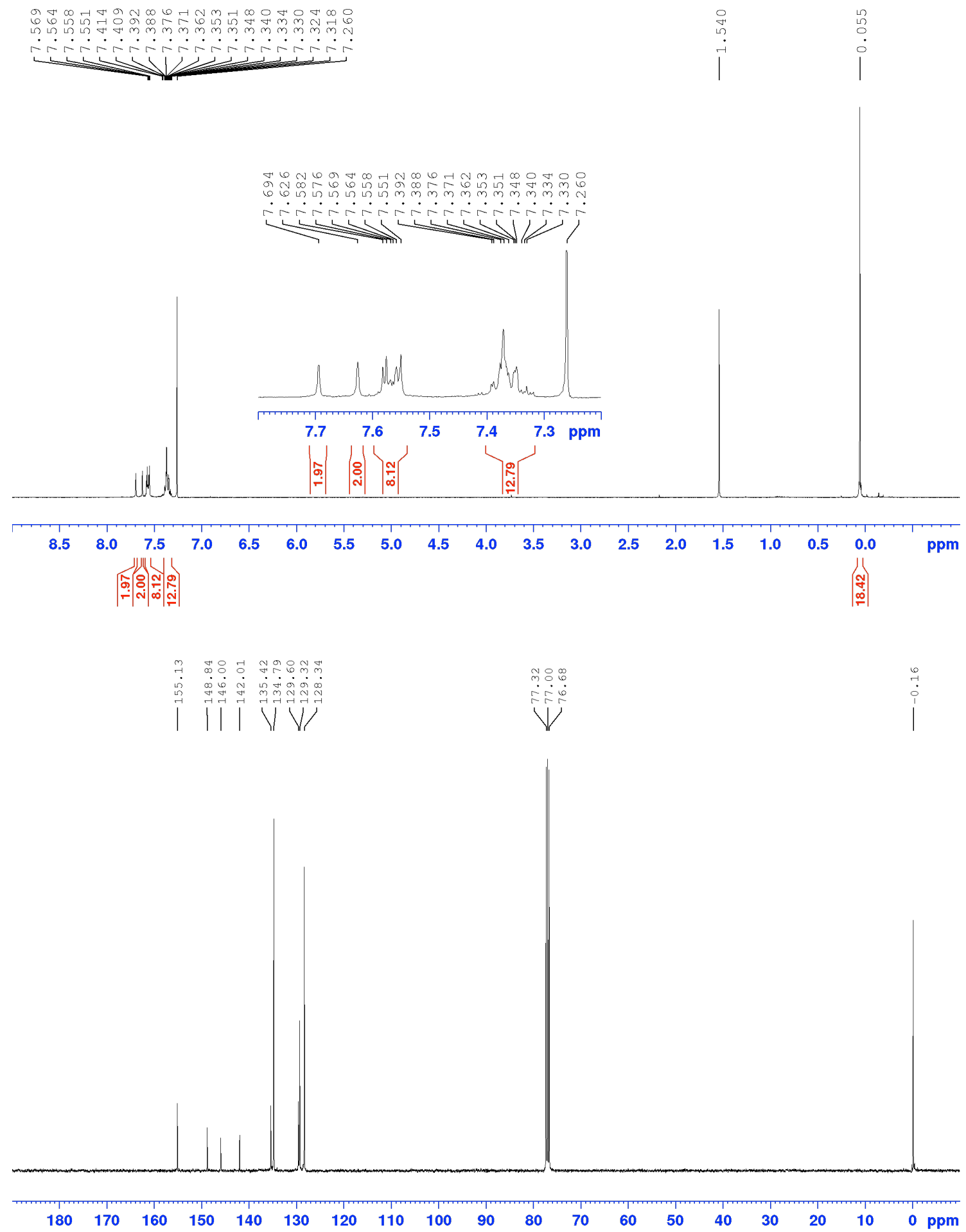
1-Bromo-2-[2-(triphenylgermyl)ethynyl]benzene.

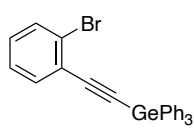

${ }^{1} \mathrm{H}$ NMR (400.13 MHz, $\left.\mathrm{CDCl}_{3}, \mathrm{CHCl}_{3}: \delta 7.26 \mathrm{ppm}\right),{ }^{13} \mathrm{C} \mathrm{NMR}\left(100.61 \mathrm{MHz}, \mathrm{CDCl}_{3}: \delta 77.0 \mathrm{ppm}\right)$
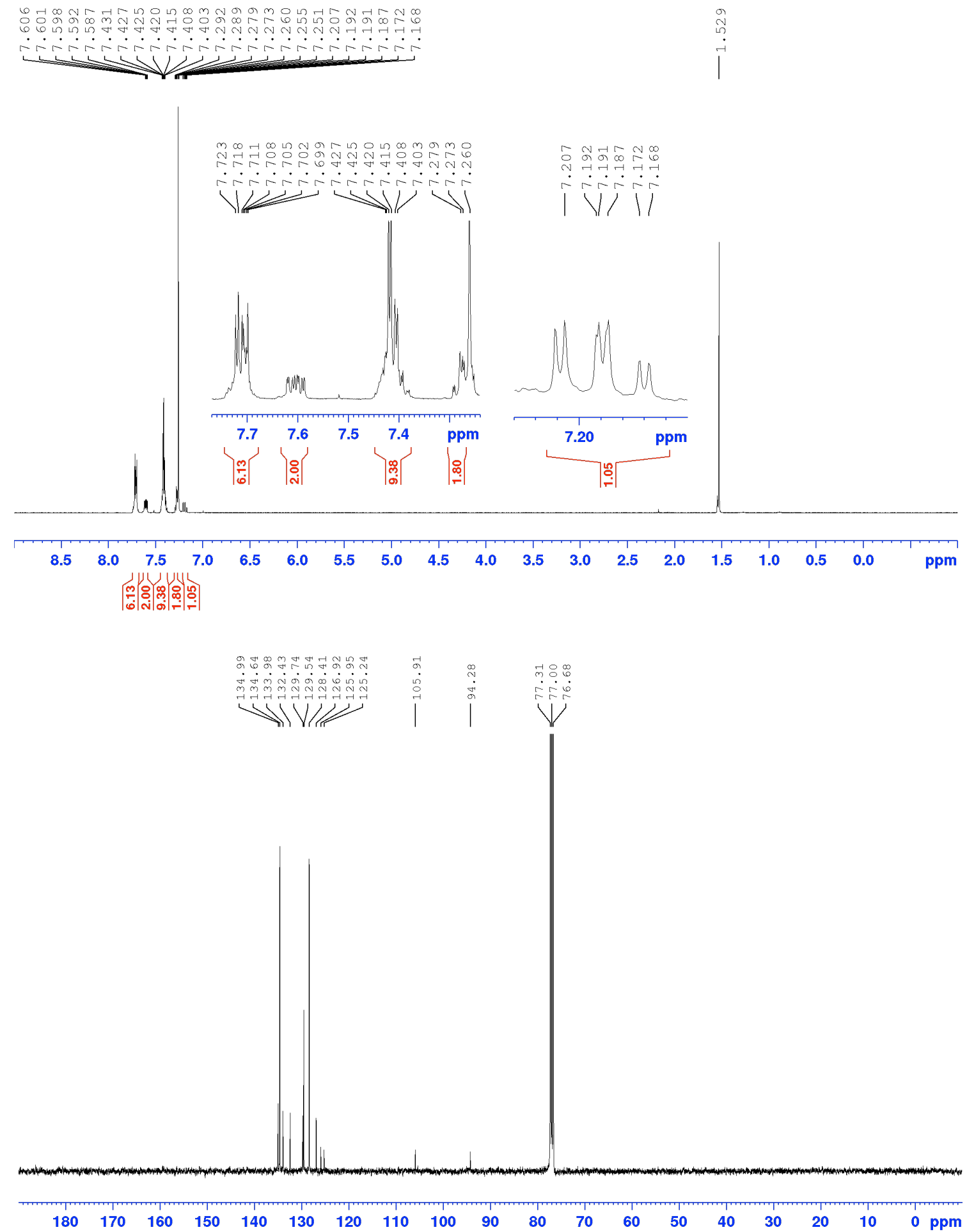


\section{2-[2-(Trimethylgermyl)ethynyl]-1-(diphenylgermyl)benzene (6a).}<smiles>COC#Cc1ccccc1[O+](c1ccccc1)c1ccccc1</smiles>

${ }^{1} \mathrm{H}$ NMR (300 MHz, $\left.\mathrm{CDCl}_{3}, \mathrm{CHCl}_{3}: \delta 7.26 \mathrm{ppm}\right),{ }^{13} \mathrm{C} \mathrm{NMR}\left(100 \mathrm{MHz}, \mathrm{CDCl}_{3}: \delta 77.0 \mathrm{ppm}\right)$
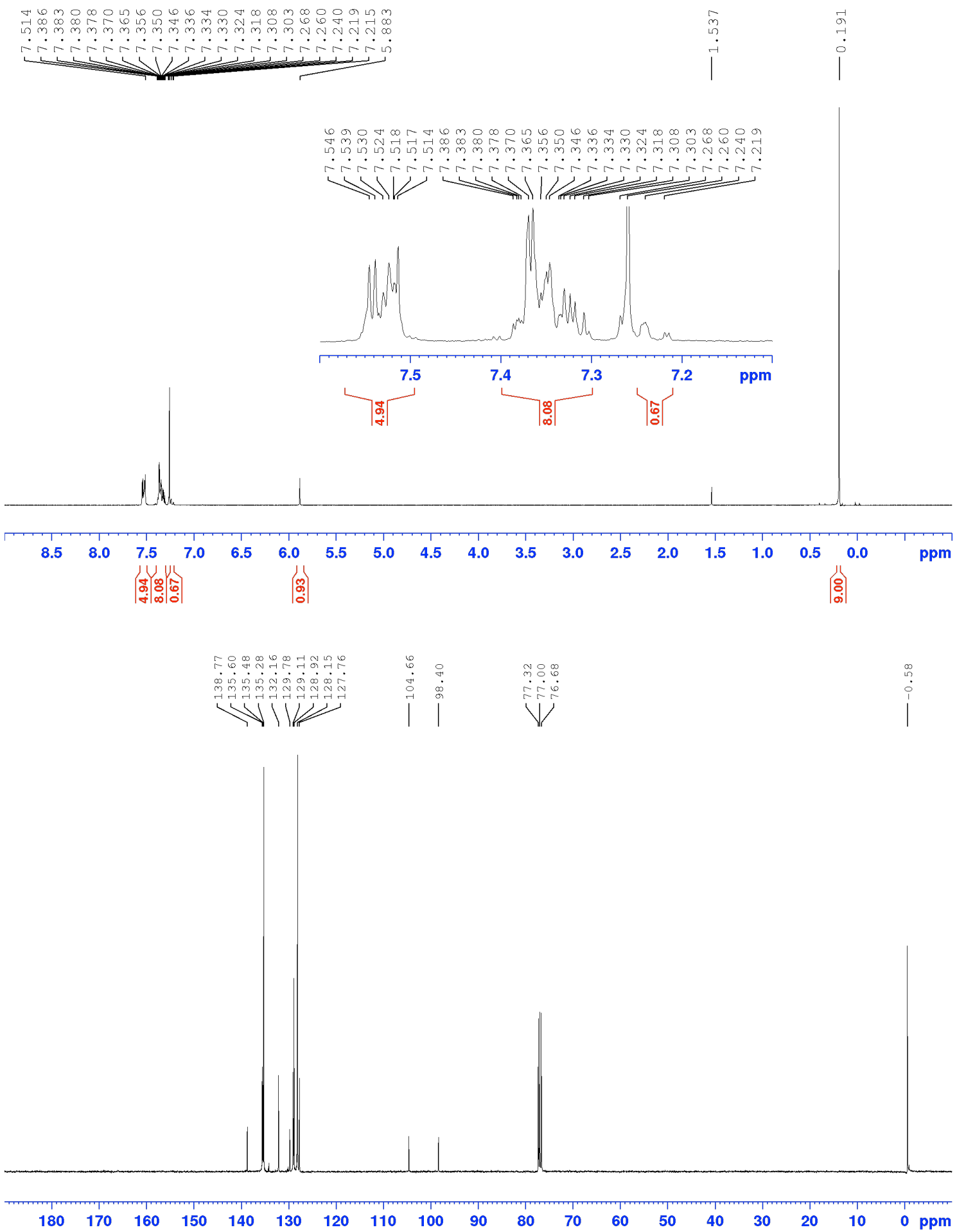
2-[2-(Triphenylgermyl)ethynyl]-1-(diphenylgermyl)benzene (6b).

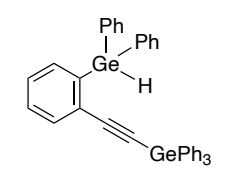

${ }^{1} \mathrm{H}$ NMR (300.13 MHz, $\left.\mathrm{CDCl}_{3}, \mathrm{CHCl}_{3}: \delta 7.26 \mathrm{ppm}\right),{ }^{13} \mathrm{C} \mathrm{NMR}\left(100.61 \mathrm{MHz}, \mathrm{CDCl}_{3}: \delta 77.0 \mathrm{ppm}\right)$
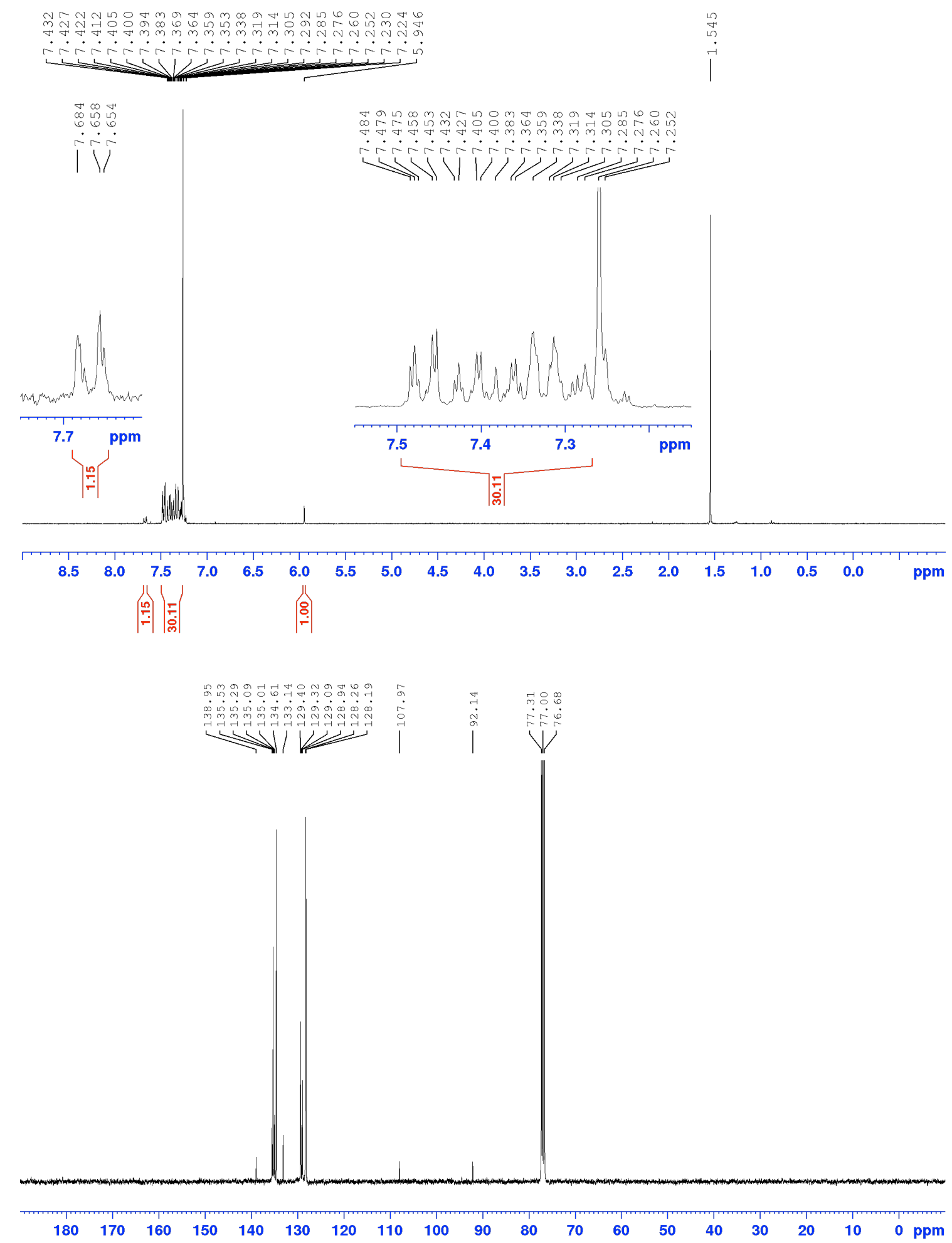


\section{1,1-Diphenyl-2-trimethylgermyl-1 $H$-benzo[b]germole (7a).}

$$
\text { (1) }{ }^{\mathrm{Ph}}
$$

${ }^{1} \mathrm{H} \mathrm{NMR}\left(400.13 \mathrm{MHz}, \mathrm{CDCl}_{3}, \mathrm{CHCl}_{3}: \delta 7.26 \mathrm{ppm}\right),{ }^{13} \mathrm{C} \mathrm{NMR}\left(100.61 \mathrm{MHz}, \mathrm{CDCl}_{3}: \delta 77.0 \mathrm{ppm}\right)$
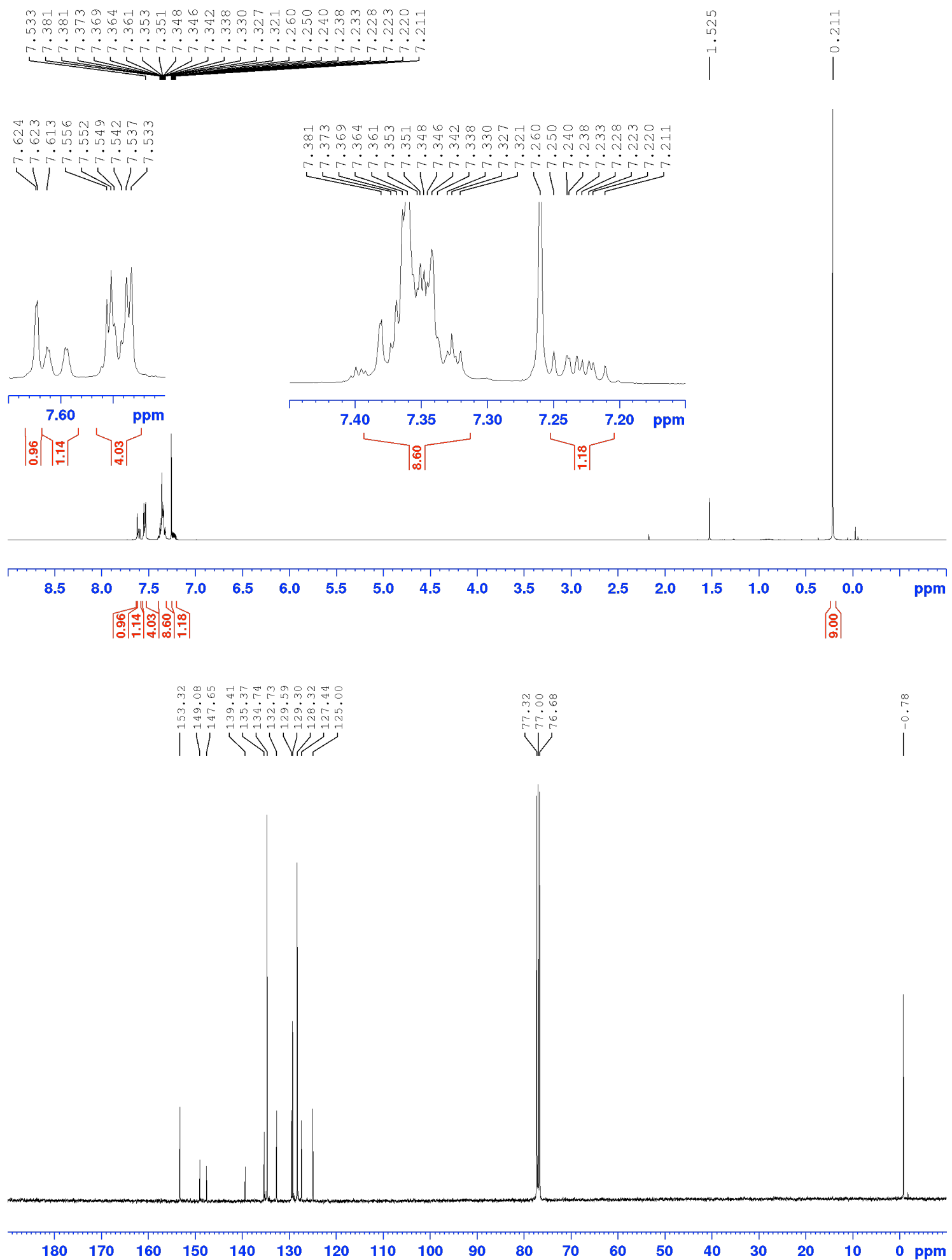
1,1-Diphenyl-2-triphenylgermyl-1H-benzo[b]germole (7b).

$$
\text { (- }{ }^{\mathrm{Ph}}
$$

${ }^{1} \mathrm{H} \mathrm{NMR}\left(400.13 \mathrm{MHz}, \mathrm{CDCl}_{3}, \mathrm{CHCl}_{3}: \delta 7.26 \mathrm{ppm}\right),{ }^{13} \mathrm{C} \mathrm{NMR}\left(100.61 \mathrm{MHz}, \mathrm{CDCl}_{3}: \delta 77.0 \mathrm{ppm}\right)$
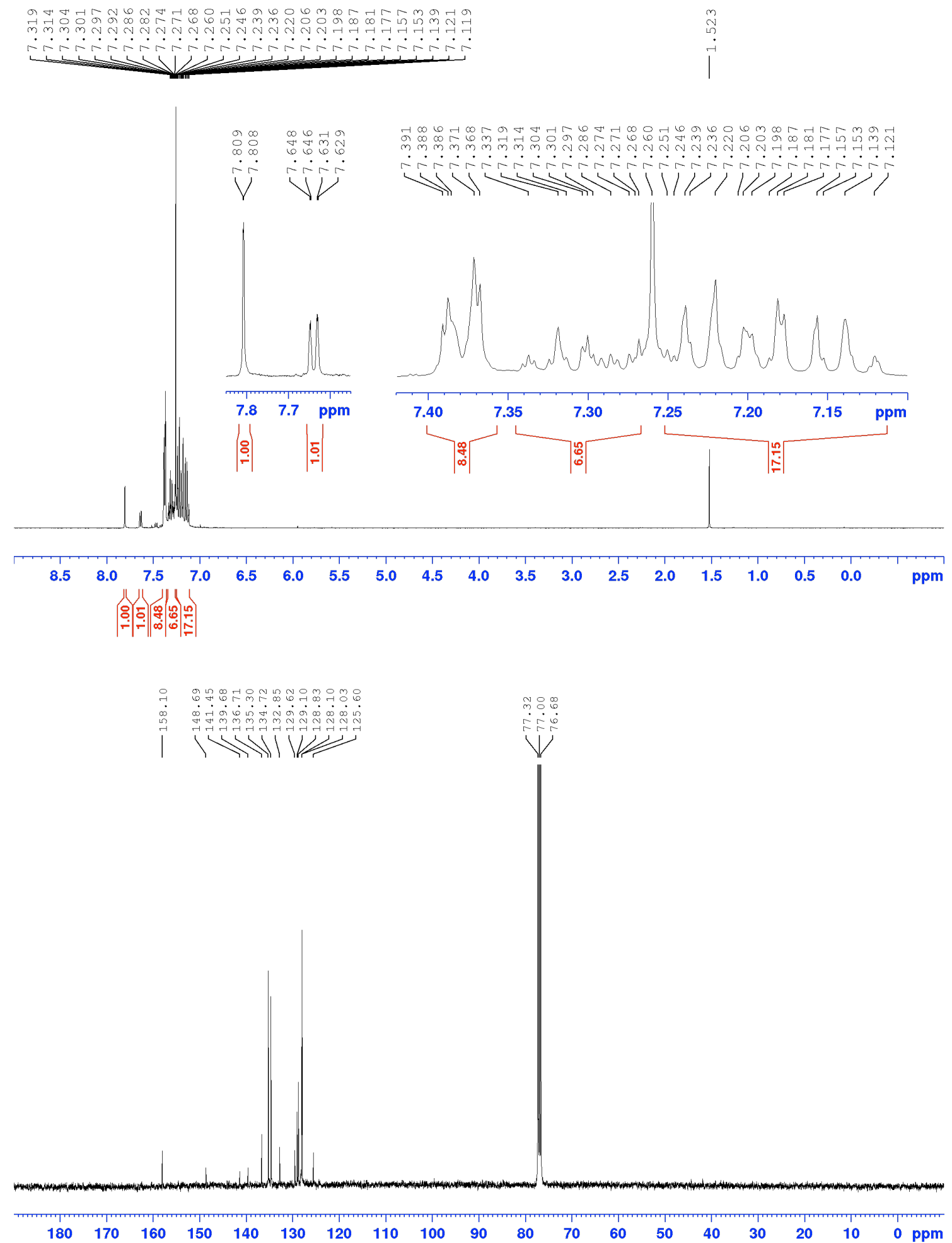
2-Iodo-1,1-diphenyl-1 $H$-benzo[b]germole (8).<smiles>IC1=Cc2ccccc2[Ge](c2ccccc2)(c2ccccc2)O1</smiles>

${ }^{1} \mathrm{H} \mathrm{NMR}\left(400.13 \mathrm{MHz}, \mathrm{CDCl}_{3}, \mathrm{CHCl}_{3}: \delta 7.26 \mathrm{ppm}\right),{ }^{13} \mathrm{C} \mathrm{NMR}\left(100.61 \mathrm{MHz}, \mathrm{CDCl}_{3}: \delta 77.0 \mathrm{ppm}\right)$
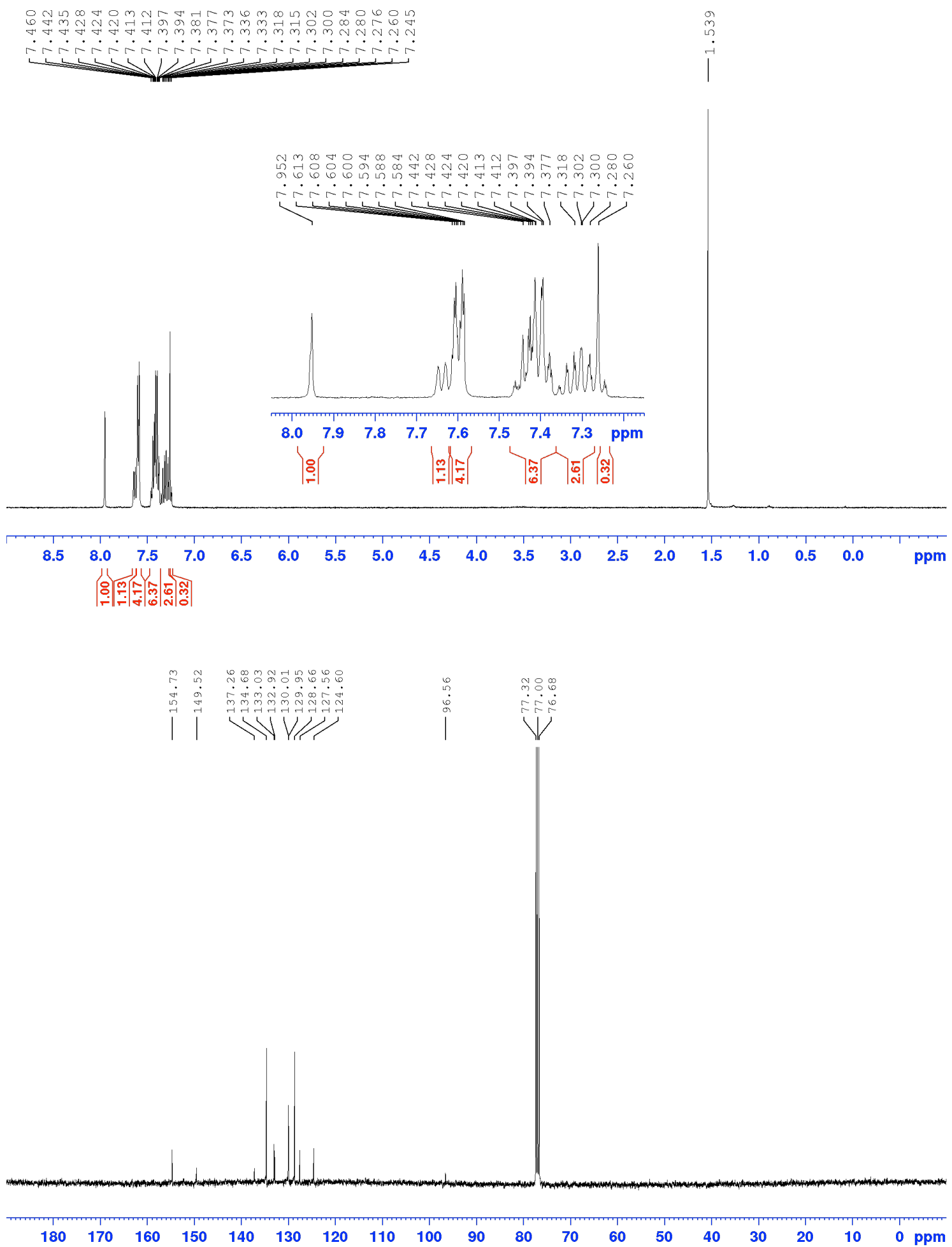